\title{
Los Alamos Benchmarks: Calculations Based on ENDF/B-V Data
}

R. B. Kidman

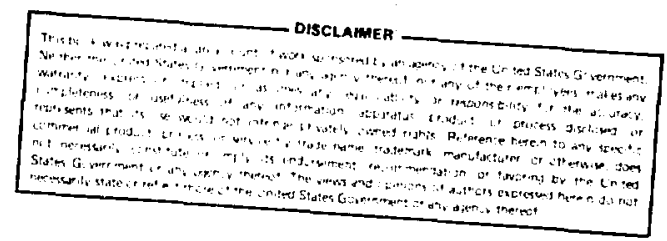


LOS ALAMOS BENCHMARKS: CALCULATIONS BASED ON ENDF/B-V DATA

by

R. B. Kidman

\section{ABSTRACT}

The new and revised benchmark specifications for nine Los Alamos National Laboratory cricical assemblies are used to compute the entire set of parameters that were measured in the experiments. A comparison between the computed and experimental values provides a measure of the adequacy of the specifications, cross sections, and physics codes used in the calculations.

\section{INTRODUCTION}

Over the years, the Los Alamos National Laboratory has constructed several small, fast critical assemblies. Numerous experimental measurements of various reactor parameters provide an opportunity to test nuclear data and computational methods. Such testing has been facilitated by the recent generation ' of simplified specifications for nine of the Los Alamos critical assemblies. Since the spherical, one-dimensional specifications are relatively simple, thay are conveniently and compactly shown in Table $I$.

This report documents our first try at using these new specifications, the latest ruclear data, and the latest computational methods to calculate all of the paraneters and compare then to the experimental results.

This nanual of Los Alamos criticals can be considered an extension of a previous report ${ }^{2}$ in which sinilar calculations were performed on 15 other benchmark assenblies.

II. PROCEDURE

The nuclear data and calculational procedures used in this study are identical to those described in Ref. 2. Very briefly, the NJOY ${ }^{3}$ code was used to 
TABLE \&

LOS ALAMUS NATLONAL LABORATORY BENCHMAKK SPECIHICATIONS

\begin{tabular}{|c|c|c|c|c|c|c|c|c|c|c|c|c|c|c|c|}
\hline \multirow{2}{*}{$\begin{array}{l}\text { Mate- } \\
\text { rial }\end{array}$} & \multirow{2}{*}{$\begin{array}{c}\text { Jezebel } \\
\text { Core }\end{array}$} & \multirow{2}{*}{$\begin{array}{c}\text { Godiva } \\
\text { Core }\end{array}$} & \multirow{2}{*}{$\begin{array}{l}\text { Jeze be } 1 \\
-23 \\
\text { Core } \\
\end{array}$} & \multirow{2}{*}{$\begin{array}{l}\text { Jezebe } 1 \\
\text {-Pu } \\
\text { Core }\end{array}$} & \multicolumn{2}{|c|}{ Bigten } & \multicolumn{2}{|c|}{ Flat top-25 } & \multicolumn{2}{|c|}{ Flat top-Pu } & \multicolumn{3}{|c|}{ Flactop-23 } & \multicolumn{2}{|l|}{ Thor } \\
\hline & & & & & Core & Refl. & Core & Ref 1 . & Core & Refl. & Core & Void & Ref 1. & Core & Ref 1. \\
\hline $\mathbf{G a}$ & $|0.001375|$ & & & 0.00138 & & & & & 0.00138 & & & & & 0.00133 & \\
\hline Th-232 & & & & & & & & & & & & & & & 0.03005 \\
\hline$u-233$ & & & 0.04671 & & & & & & & & 0.04671 & & & & \\
\hline$u-235$ & & 0.04500 & 0.00001 & & 0.00484 & 0.00010 & 0.04449 & 0.00034 & & 0.00034 & 0.00001 & & 0.00034 & & \\
\hline$u-238$ & & $0.002 i, 98$ & 0.00029 & & 0.04268 & 0.04797 & 0.00270 & 0.04774 & & 0.04774 & $0.00028 \mid$ & & 0.04774 & & \\
\hline Pu-239 & 0.03705 & & & 0.02994 & & & & & 0.03674 & & & & & 0.03618 & \\
\hline$P u-240$ & 0.001751 & & & 0.00788 & & & & & 0.00186 & & & & & 0.00194 & \\
\hline Pu-242 & & & & 0.00016 & & & & & & & & & & & \\
\hline $\begin{array}{l}\text { Radiug } \\
(\mathrm{cm})\end{array}$ & 6.385 & 8.741 & 5.983 & 6.599 & 30.48 & 45.72 & 6.116 & 24.13 & 4.533 & 24.13 & 4.317 & 4.610 & 24.13 & 5.310 & 29.88 \\
\hline $\begin{array}{c}\text { Inter- } \\
\text { vals }\end{array}$ & 40 & 40 & 40 & 40 & 20 & 40 & 30 & 30 & 30 & 30 & 30 & 2 & 30 & 30 & 30 \\
\hline
\end{tabular}

aDensities given in units of atoms/b-cu, 
generate 70-group cross sections from ENDF/B-V data. ${ }^{4}$ Self-shielded, effective cross sections for each benchmark were generated by running the $70-$ group cross sections through SUPERX. [SUPERX is an unpublished, overhauled version of IDX $^{5}$ that Incorporates the following changes: (1) improved f-factor interpolation; (2) Improved elastic downscatter iteration; (3) Inclusion of elastic scattering transfer matrices; (4) inclusion of elastic downscatter f-factors; (5) improved transport cross-section computation; (6) addition of isotope and regiondependent fission source matrices.] Fluxes and adjoints from SUPERX were fed into SUPERB for the calculation of diffusion theory material worths and fluxes and adjoints from $P_{1 / 2} S_{16}$ ONETRAN $^{6}$ runs were fed into SUPERB to generate transport theory material worths. [SUPERB is an unpublished, overhauled version of PERTV ${ }^{7}$ that can accept cross sections, isotopes, and region-dependent fission source matrices from SUPERX.] The group velocities and delayed neutron data used by SUPERB are presented in Appendix A of Ref. 2 .

\section{RESULTS}

All of the computed results and comparisons with every experimental measurement are compiled in Appendix A. Although transport theory results are more sound, diffusion theory parameter results are also shown as a matter of passing interest. However, all of the fluxes, adjolnts, fission sources, and average cross sections tabulated for each benchmark are $\mathrm{P}_{1 / 2} \mathrm{~S}_{16}$ transport theory

We wish to convert the $\mathrm{P}_{1 / 2} \mathrm{~S}_{16}$ transport theory eigenvalues of Appendix A to $P_{\infty} S_{\infty}$ estimates. Eigenvalue behaviors vs $P_{i}$ and vs $S_{i}$ are shown in Tables II and III, respectively. These results were obtained using ONEDANT, 8 infinitely dilute cross sections, and a single vector fission source for each benchmark. The $P_{\infty}$ eigenvalue estimates were obtained by continuing the same fractional decreases exhibited by $\left(K_{3}-K_{4}\right) /\left(K_{4}-K_{5}\right)$, on out to infinity. The $S_{\infty}$ estimate was obtained from a Lagrange 5-point interpolation scheme on the points $\left(1 / i, K_{i}\right)$. Now, if one assumes that computing the $P_{i}$ eigenvalues at $S_{i \neq 16}$, or computing the $S_{1}$ eigenvalues at $P_{1 \neq 3}$, or that improvements like self-shielding or fission source matrices will not change the eigenvalue behavior significantly, then the results in Tables II and III can be used to convert the more refined $P_{1 / 2} S_{16}$ eigenvalues of Appendix $A$ to $P_{\infty} S_{\infty}$ results. This is done in Table IV.

The calculations of Appendix A made use of the transport theory code ONETRAN, which used cross sections processed with SUPERX. ONETRAN was used in place 
of the more recent version, ONEDANT, because ONETRAN has the capability to use the region-dependent fission source matrices from SUPFRX. But SUPERX is an overhauled version of $10 X$ containing many changes. Since one frequently wishes to know how certain changes may affect his results, estimates of how the above changes affect the eigenvalues are shown in Table $V$. The column labeled "Elastic Matrix" indicates the elgenvalue change one obtalns by using an elastic scattering matrix in SUPERX rather than just using one elastic outscatter term as in IDX. The column labeled "Chi Matrix" displays mainly the effect of computing with a chi matrix rather than just using a single chi vector. This column also includes smaller effects caused by self-shielding and spectral adjustment of elastic downscatter. The next column shows how the eigenvalue decreases when computing with ONETRAN rather than with ONEDANT. The last column shows the sum of these effects which are incorporated in the Appendix A results.

Other reactor parameters also respond to the above code changes. Table VI lists the $\mathrm{C} / \mathrm{E}$ (calculated/experimental) changes to a few selected central parameters. These changes should be compared to the corresponding $C / E$ values in Appendix $A$ to get a more balanced picture of the changes involved.

The computer run times for most of the code submittals of this study are shown in Appendix $B$.

IV. DISCUSSION

Previous $\mathrm{P}_{1 / 2} \rightarrow \mathrm{P}_{\infty}$ and $\mathrm{S}_{16} \rightarrow \mathrm{S}_{\infty}$ corrections ${ }^{9}$ for Jezebel and Godiva summed to -0.0053 and -0.0047 , respectively. The corresponding results from this study are -0.0034 and -0.0039 , respectively. Obviously, such corrections are not constant across varying cross sections and changing methods. The most probable cause for the above differences is the change in cross sections (ENDF/B-IV ENDF/B-V). However, eigenvalue behavior (and hence, the corrections) could change slightly if it were determined at different angular quadratures and Legendre orders, or if self-shielding and fission source matrices were used. Also, more suitable extrapolation schemes could yield slightly different $P_{\infty}$, and $S_{\infty}$ eigenvalue estimates. $P_{\infty}$ and $S_{\infty}$ corrections become less important for larger systems (note the relatively small corrections for Bigten).

The methods improvements coded into SUPERX and SUPERB are beneficial for these nine benchmarks. According to Tables IV and V, only the Jezebe1-23 and Jezebel-Pu eigenvalues suffered as a result of the code changes. The Godiva 
TABLE II

EIgenVALUe BehavioR VS $P_{i}$ (ALL $s_{16}$ )

\begin{tabular}{|c|c|c|c|c|c|c|c|c|}
\hline & $\mathbf{P}_{0}$ & $P_{1 / 2}$ & $\mathrm{p}_{1}$ & $P_{2}$ & $\mathbf{P}_{3}$ & $P_{4}$ & $P_{5}$ & $\mathbf{P}_{\infty}$ \\
\hline JEZEBEL & 1.009107 & 1.012750 & 1.003351 & 1.009515 & 1.009407 & 1.009411 & 1.009410 & 1.009410 \\
\hline GODIVA & 1.111715 & 1.004524 & 0.995863 & 1.001335 & 1.001287 & 1.001293 & 1.001291 & 1.001292 \\
\hline JEZEBEL-23 & 1.104118 & 0.998695 & 0.987885 & 0.994773 & 0.994688 & 0.994692 & 0.994691 & 0.994691 \\
\hline JEZEBEL-PU & 1.092024 & 1.004189 & 0.994834 & 1.000952 & 1.000852 & 1.000856 & 1.000855 & 1.000855 \\
\hline BIGTEN & 1.065059 & 1.012091 & 1.010722 & 1.011627 & 1.011591 & 1.011595 & 1.011594 & 1.011594 \\
\hline FLATTOP -25 & 1.146298 & 1.017309 & 0.994933 & 1.012890 & 1.009223 & 1.010013 & 1.009849 & 1.009877 \\
\hline FLATTOP-Pu & 1.159393 & 1.023888 & 0.990812 & 1.019677 & 1.011958 & 1.013995 & 1.013496 & 1.013594 \\
\hline FLATTOP-23 & 1.148283 & 1.016788 & 0.985888 & 1.013658 & 1.005577 & 1.007753 & 1.007196 & 1.007310 \\
\hline THOR & 1.151387 & 1.028662 & 0.991730 & 1.026523 & 1.015258 & 1.018901 & 1.017836 & 1.018077 \\
\hline
\end{tabular}

TABLE III

EIgENVALUE BEHAVIOR VS $s_{i}$ (ALL $P_{3}$ )

\begin{tabular}{lccccccc} 
& \multicolumn{2}{c}{$\mathrm{S}_{4}$} & $\mathrm{~S}_{8}$ & $\mathrm{~S}_{16}$ & $\mathrm{~S}_{32}$ & $\mathrm{~S}_{48}$ & $\mathrm{~S}_{\infty}$ \\
\cline { 2 - 3 } & 1.021764 & 1.012103 & 1.009407 & 1.008665 & 1.008522 & 1.008410 \\
GEDEBEL & 1.009787 & 1.003115 & 1.001287 & 1.000778 & 1.000679 & 1.000601 \\
JEZEBEL-23 & 1.005988 & 0.997147 & 0.994688 & 0.994009 & 0.993878 & 0.993776 \\
JEZEBEL-Pu & 1.012844 & 1.003465 & 1.000852 & 1.000131 & 0.999992 & 0.999884 \\
BIGTEN & 1.012258 & 1.011723 & 1.011591 & 1.011555 & 1.011548 & 1.011542 \\
FLATTOP-25 & 1.021648 & 1.011491 & 1.009223 & 1.008612 & 1.008494 & 1.008404 \\
FLATTOP-Pu & 1.030267 & 1.015254 & 1.011958 & 1.011072 & 1.010900 & 1.010766 \\
FLATTOP-23 & 1.022704 & 1.008602 & 1.005577 & 1.004737 & 1.004561 & 1.004383 \\
THOR & 1.035998 & 1.019100 & 1.015258 & 1.014254 & 1.014048 & 1.013827
\end{tabular}


TABLE IV

BEST EIGENVALUE EST IMATES

Uncorrected

$\mathrm{p}_{1 / 2} \mathrm{~s}_{16}$

Eigenvalue
$\mathrm{P}_{1 / 2} \rightarrow \mathrm{P}_{\infty}$

$\mathrm{S}_{16} ; \mathrm{S}_{\infty}$

Correction Correction
Corrected

$P_{\infty} S_{\infty}$

Eigenvalue

$\begin{array}{lllll}\text { JEZEBEL } & 1.0111 & -0.0033 & -0.0010 & 1.0068 \\ \text { GODIVA } & 1.0028 & -0.0032 & -0.0007 & 0.9989 \\ \text { JEZEBEL-23 } & 0.9983 & -0.0040 & -0.0009 & 0.9933 \\ \text { JEZEBLL-Pu } & 1.0023 & -0.0033 & -0.0010 & 0.9980 \\ \text { BIGTEN } & 1.0107 & -0.0005 & -0.0000 & 1.0101 \\ \text { FLATTOP-25 } & 1.0149 & -0.0074 & -0.0008 & 1.0067 \\ \text { FLATTOP-PU } & 1.0207 & -0.0103 & -0.0012 & 1.0093 \\ \text { FLATTOP-23 } & 1.0145 & -0.0095 & -0.0012 & 1.0038 \\ \text { THOR } & 1.0266 & -0.0106 & -0.0014 & 1.0146\end{array}$

TABLE V

E IGENVALUE EFEECTS OF CODE Changes

\begin{tabular}{llll} 
Elastic & Chi & ONETRAN & Sum of \\
Matrix & Matrix & -ONEDANT & Effects \\
\hline
\end{tabular}

\begin{tabular}{|c|c|c|c|c|}
\hline JEZEBEL ${ }^{a}$ & -0.0001 & -0.0015 & -0.0001 & -0.0017 \\
\hline GODIVA & -0.0004 & -0.0016 & -0.0001 & -0.0021 \\
\hline JEZEBEL-23 & -0.0001 & -0.0003 & -0.0001 & -0.0005 \\
\hline JEZEBEL-Pu & -0.0000 & -0.0017 & -0.0001 & -0.0019 \\
\hline B IGTEN ${ }^{2}$ & -0.0046 & -0.0014 & -0.0001 & -0.006 \\
\hline FLATTOP $-25^{a}$ & -0.0034 & -0.0021 & -0.0003 & -0.0058 \\
\hline FLATTOP-Pu ${ }^{a}$ & -0.0034 & -0.0026 & -0.0006 & -0.0065 \\
\hline FLATTOP $-23^{\mathrm{a}}$ & -0.0031 & -0.0017 & -0.0006 & -0.0054 \\
\hline THOR & -0.0022 & -0.0016 & -0.0004 & -0.0042 \\
\hline
\end{tabular}

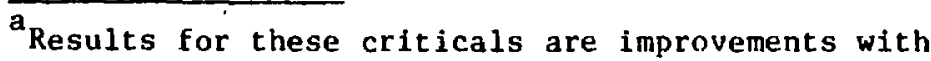
respect to experimental measurements. 
TABLE VI

SOME CENTRAL PARAMETER C/E EFFECTS dUE TO ALL CODE CHANGES

\begin{tabular}{|c|c|c|c|c|c|c|c|c|c|}
\hline & $\frac{\sigma_{f}^{U-238}}{\sigma_{f}^{U-235}}$ & $\frac{\sigma_{f}^{U-233}}{\sigma_{f}^{U-235}}$ & $\frac{\sigma_{f}^{N p-237}}{\sigma_{f}^{U-235}}$ & $\frac{\sigma_{f}^{P u-239}}{\sigma_{f}^{U-235}}$ & $\begin{array}{l}\text { Be-9 } \\
\text { Worth }\end{array}$ & $\begin{array}{l}\text { Fe } \\
\text { Worth }\end{array}$ & $\begin{array}{l}\text { U-235 } \\
\text { Worth }\end{array}$ & $\begin{array}{l}\text { Pu-239 } \\
\text { Worth }\end{array}$ & $\begin{array}{l}\text { Void } \\
\text { Worth }\end{array}$ \\
\hline JEZEBEL & -0.0068 & $0.0005^{a}$ & $0.0011^{a}$ & $0.0006^{a}$ & $-0.2447^{a}$ & $-0.0497^{a}$ & $-0.0038^{a}$ & 0.0045 & - \\
\hline GODIVA & 0.0021 & 0.0000 & 0.0049 & $0.0010^{a}$ & $0.1298^{a}$ & $-0.9783^{a}$ & $0.0042^{a}$ & $0.0073^{a}$ & $-\infty$ \\
\hline JEZEBEL,-23 & -0.0071 & - - & $0.0026^{a}$ & $-\infty-\infty$ & ------ & $-\infty-\infty$ & ---- & ----- & $0.0060^{\mathrm{a}}$ \\
\hline JEZEBEL-Pu & -0.0083 & $-\infty-\infty$ & 0.0003 & ------ & $-\infty$ & --- & ---- & ----- & - \\
\hline B IGTEN & $-0.0018^{a}$ & $0.0001^{a}$ & 0.0047 & 0.0000 & 0.1680 & $-0.0321^{a}$ & 0.0015 & 0.0185 & \\
\hline FLATTOP-25 & 0.0035 & -0.0001 & 0.0061 & 0.0011 & ------ & $-\infty--$ & $0.0106^{a}$ & $0.0145^{a}$ & --ー-- \\
\hline FLATTOP-Pu & $0.0081^{a}$ & -- & 0.0072 & - & - & - & $0.0138^{\mathrm{a}}$ & $0.0220^{\mathrm{a}}$ & - \\
\hline FLATTOP-23 & $0.0023^{a}$ & ---- & 0.0016 & ----- & ----- & $-\infty--$ & $-\cdots$ & ---- & $0.0193^{a}$ \\
\hline THOR & -0.0068 & --- & $0.0018^{a}$ & - - - & ------ & --- - & ------ & ------ & 0.0120 \\
\hline
\end{tabular}

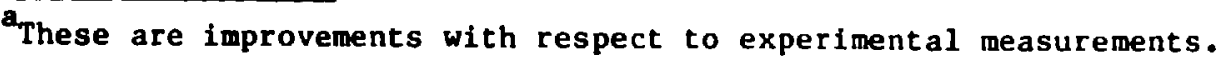


eigenvalue is no worse off whereas the remaining six elgenvalues reflect definite Improvements. About $60 \%$ of all the parameter $\mathrm{C} / \mathrm{E}$ changes (some of which are featured in Table VI) were improvements.

Past hopes were that introducing elastic scattering matrices and elastic downscater f-factors would improve light element worth calculations to the same level as heavy element worth calculations. That did not happen. Even though light element calculated worths were helped the most, they still remain spurious, long distances from the experimental values. On the other hand, the elastic matrix changes produced much larger effects in the reflected assembly eigenvalues than expected (see Table $V$ ).

The introduction of isotope- and region-dependent fission source matrices produced disappointingly small eigenvalue effects (see Table $V$ ). This is surprising since the change from a single vector chi for each assembly to regiondependent chi matrices is quite substantial. Some of the heavy element central worths were directly affected by this change because their worths are now computed using their own unique isotope chi matrix rather than using some sort of globally/compositionally averaged vector chi.

If one ignores for the moment the Jezebel elgenvalue in Table IV, the reflected assembly eigenvalues appear to be systematicaily high. The problem was addressed previously, ${ }^{10}$ at which point it was found that higher Legendre order cross sections $\left(>P_{3}\right)$ and chi matrices were required (as was done in this study) for adequate calculation, although this did not solve the problem. That study also suggested that improved clean specifications would not solve the problem and one would have to resort to cross-section adjustment. This study does not change that conclusion. However, the relatively large elastic matrix effects registered by the reflected assemblies (see Talie V) suggest that perhaps the elastic/inelastic scattering matrices of the involved heavy elements should be scrutinized.

Although one always desires a better match between theory and experiment, the results of this study can be considered more a validation than a nullification of the specifications, cross sections, and codes. 


\section{REFERENCES}

1. Los Alanos National Laboratory internal memorandum, Q-14-79-314, from G. E. Hansen and H. C. Paxton to R. J. LaBauve, concerning Fast Critical Specifications in Benchmark Format, 18 October 1979.

2. R. B. Kidman, "ENDF/B-V, LIB-V, and the CSEWG Benchmarks," Los Alamos National Laboratory report LA-8950-MS (ENDF-314) (August 1981).

3. R. E. MacFarlane, R. J. Barrett, D. W. Muir, and R. M. Boicourt, "The NJOY Nuclear Data Processing System: User's Manua1," Los Alamos Scientific Laboratory report LA-7584-M (December 1978).

4. D. Garber, Ed., "Data Formats and Procedures for the ENDF Neutron Cross Section Library," Brookhaven National Laboratory report BNL 50274 (1976).

5. R. W. Hardie and W. W. Little, Jr., "1DX, A One-Dimensional Diffusion Code for Generating Effective Nuclear Cross Sections," Battelle Northwest Laboratory report BNWL-1162 (1969).

6. T. R. Hill, "ONETRAN: A Discrete Ordinates Finite Element Code for the Solution of the One-Dimensional Multigroup Transport Equation," Los Alamos Sclentific Laboratory report LA-5990-MS (June 1975).

7. R. W. Hardie and W. W. Little, Jr., "PERT-V, A Two-Dimensional Perturbation Code for Fast Reactor Analysis," Battelle Northwest Laboratory report BWNL-1162 (1969).

8. R. D. O'De11, F. W. Brinkley, Jr., D. R. Marr, "A Code Package for One-Dimensional Diffusion-Accelerated, Neutra1-Particle Transport," Los Alanos National Laboratory report to be published.

9. E. M. Bohn, Ed., "Benchmark Testing of ENDF/B-IV," Brookhaven National Laboratory report BNL-NCS-2118 (ENDF-230) (1976).

10. R. B. Kidman, "Reckoning THOR," Los Alamos National Laboratory report LA-8856-MS (May 1981). 
APPENDIX A

LOS ALAMOS NATIONAL LABORATORY BENCHMARK RESULTS

This appendix contains the one-dimensional diffusion theory and onedimensional $\mathrm{P}_{1 / 2} \mathrm{~S}_{16}$ transport theory results for each of the nine Los Alamos National Laboratory benchmarks. Tables A-I through A-IX compare calculated results to the available experimental results.

Tables A-X through A-XXXVII show the 70-group and collapsed 25-group transport theory central fluxes, central adjoints, edge fluxes, edge adjoints, and region fission fractions. The fluxes are normalized so that the central flux sum is 1.0. The adjoints are normalized so that the sum of the product of the central adjoint and the central effective fission source is 1.0 . The reactor fission fraction vectors were obtained by computing the total number of $\nu \sigma_{f} f$ neutrons born into each group and then normalizing so that the sum over all groups would be 1.0 . The 25 -group adjoints were obtained by a simple addition of the original fine-group adjoints in each coarse group.

Tables A-XXXVIII through A-XLVI contain several centrally averaged onegroup cross sections. The fission and "capture" cross sections are simply flux averaged. |Actually, "capture" = capture $-(n, 2 n)-2 *(n, 3 n)$. However, if the central reaction rate ratios called for an isotope's capture or $(n, y)$ reaction rate, then efforts were taken to make "capture" = capture.l Nu is $\emptyset_{f}$ averaged, and the transport cross section is $\left(\emptyset_{1}-\phi_{2}\right) / \Sigma_{\text {tr }}$ averaged (where $\emptyset_{i}$ is the flux at mesh point $i$, and $\varepsilon_{t r}$ is the macroscopic transport cross section).

Several symbols used in the tables are defined below.

\% Uncer $=$ per cent error on measured values

$A(/ s) \quad=$ Rossi Alpha in units of $1 / 5$

$\mathrm{S} \quad=$ seconds

Beta $=$ effective delayed neutron fraction

E $=$ exponent to the base 10 follows

$\mathrm{L} \quad=$ neutron ggeneration time

R/IH $\quad=$ reactivity/in-hour conversion factor

\$ = dollar, another unit of reactivity

$\mathrm{kg} \quad \mathrm{kilogram}$

$\mathrm{C} / \mathrm{E} \quad=$ calculated/experimental ratio 
Fij = fission reaction rate for the material with atomic number ending with 1 and atomic mass number ending with $j$

Gif $=(n, y)$ reaction rate

A sym $\quad=(n, \alpha)$ reaction rate for material sym

$2 n$ sym $=(n, 2 n)$ reaction rate for material sym

$\mathrm{n} / \mathrm{cm}^{2} / \mathrm{s}=$ neutrons per square centimeter per second

rho/n/s = reactivity gain per neutron insertio per second

$\mathrm{eV} \quad=$ electron volts

The measured worths reported in the specifications have all been converted to units of $\$ / \mathrm{kg}$ for this study. Normally, this conversion requires knowledge of the effective delayed neutron fraction ( $\beta$ ). If $\beta$ was not explicitly specified, it was inferred from some material worth (for example, ${ }^{235} \mathrm{U}$ ) that was specified in units of $\delta \mathrm{K} / \mathrm{K} / \mathrm{g} \cdot$ atom but was measured in units of $\$ / 8 \cdot a t o m$. 


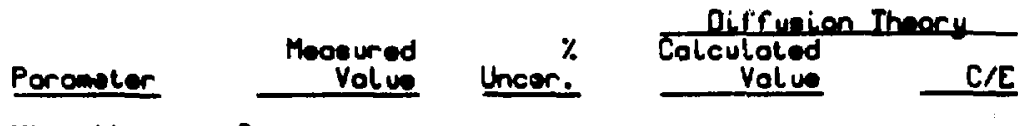

Minallonoun Porometere

Eigenvolu

A $(15)$

Beto

L (S)

R/IH

$\begin{array}{rr}0.20 & 9.600 E-01 \\ 1.56 & -5.224 E+05 \\ 1.56 & 1.873 E-03 \\ 1.56 & 3.583 E-09 \\ & 7.489 E-06\end{array}$

0.9500

0.8163

0.9853

1. 2059

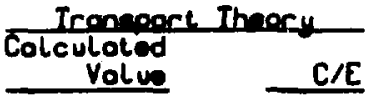

$1.011 E+00$
$-5.204 E+05$
$1.861 E-03$
$3.582 E-09$
$7.461 E-05$

1.0111

0.8131

0.9211

1.2005

\section{Centrol Reaction Rote Ratios}

\begin{tabular}{|c|c|c|c|c|c|c|}
\hline $\begin{array}{l}\text { F28/F25 } \\
F 23 / F 25 \\
F 37 / F 25 \\
\text { F } 49 / F 25 \\
\text { GV/F25 } \\
\text { GHNS5/F25 } \\
\text { GCU/F } 25 \\
\text { GNB93/F25 } \\
\text { EAU197/F25 }\end{array}$ & $\begin{array}{l}2.137 E-01 \\
1.576 E+00 \\
9.620 E-01 \\
1.418 E+00 \\
2.300 E-03 \\
2.400 E-03 \\
1.000 E-02 \\
2.300 E-02\end{array}$ & $\begin{array}{r}1.08 \\
1.71 \\
1.66 \\
2.00 \\
13.04 \\
12.50 \\
6.00 \\
8.70\end{array}$ & $\begin{array}{l}1.925 E-01 \\
1.557 E+00 \\
9.402 E-01 \\
1.404 E+00 \\
1.818 E-03 \\
2.095 E-03 \\
8.498 E-03 \\
2.767 E-02\end{array}$ & $\begin{array}{l}0.9010 \\
0.9869 \\
0.9774 \\
0.9698 \\
0.7902 \\
1.2064 \\
0.8498 \\
1.2031\end{array}$ & $\begin{array}{l}1.959 E-01 \\
1.557 E+00 \\
9.515 E-01 \\
1.408 E+00 \\
1.782 E-03 \\
2.838 E-03 \\
8.379 E-03 \\
2.712 E-02\end{array}$ & $\begin{array}{l}0.9169 \\
0.9065 \\
0.9091 \\
0.9724 \\
0.7750 \\
1.1826 \\
0.8379 \\
1.1791 \\
0.9530\end{array}$ \\
\hline
\end{tabular}

Central Reactivity Worths 15/kol

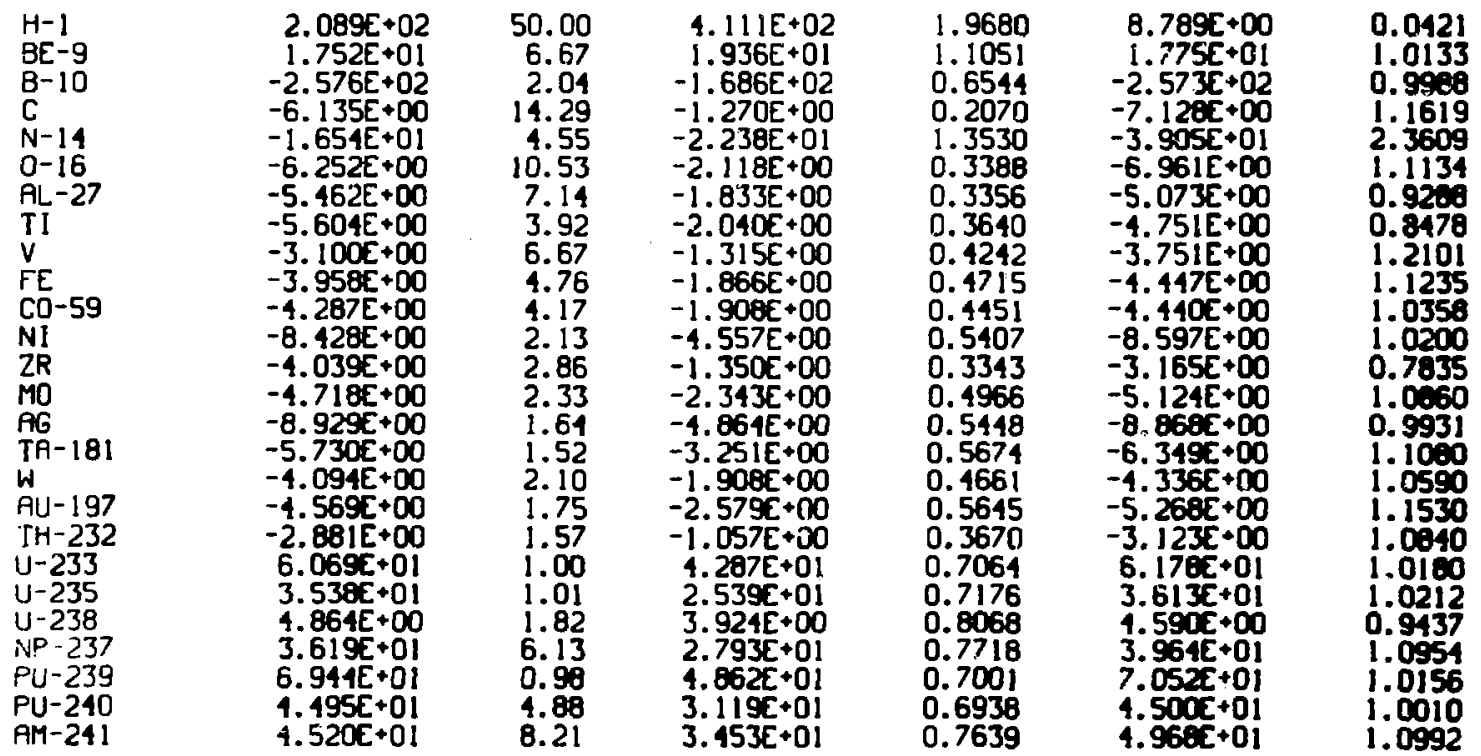

Jezebei Leokage Flux Comporison

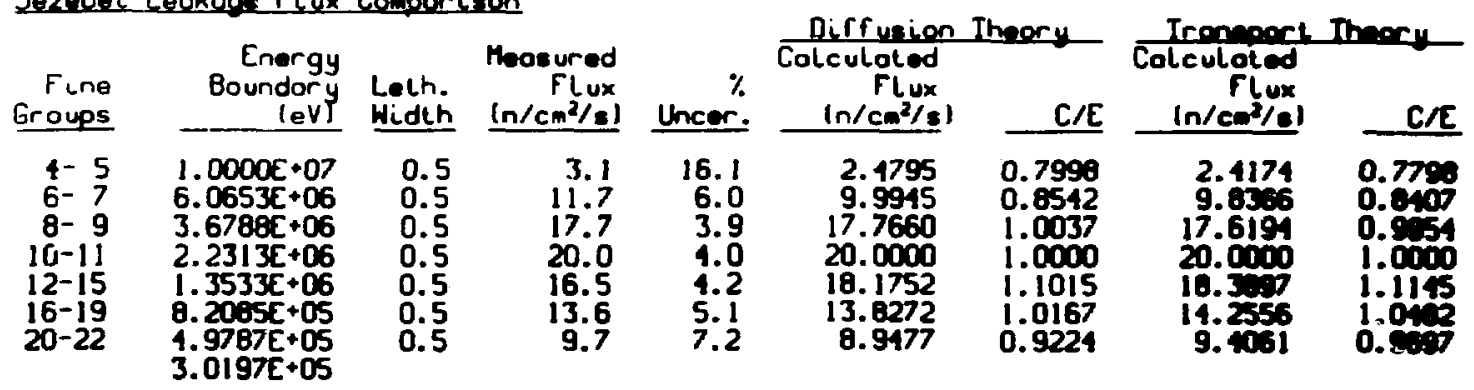


TRaLE A-II

GOOIVA RESULTS

\begin{tabular}{|c|c|}
\hline orometer & $\begin{array}{l}\text { Moosured } \\
\text { Volue }\end{array}$ \\
\hline \multicolumn{2}{|c|}{ Misenllomoun Poromtere } \\
\hline $\begin{array}{l}\text { igenvalue } \\
\text { (/SI } \\
\text { to } \\
\text { [S] } \\
\text { [H }\end{array}$ & $\begin{array}{r}1.000 E+00 \\
-1.110 E+06 \\
6.450 E-03 \\
5.811 E-09\end{array}$ \\
\hline
\end{tabular}
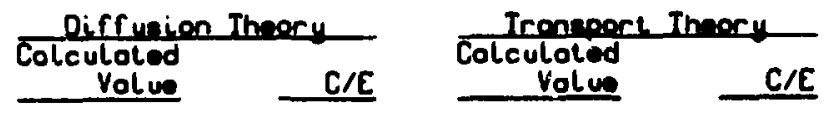

\section{Centroil Reaction Role Rotios}

$\begin{array}{lll}\text { F28/F25 } & 1.547 E-01 & 1.09 \\ \text { F23/F25 } & 1.590 E+00 & 1.89 \\ \text { F37/F25 } & 8.370 E-01 & 1.55 \\ \text { F49/F25 } & 1.402 E+00 & 1.78 \\ \text { GHN55/F25 } & 2.700 E-03 & 7.41 \\ \text { 6C059/F25 } & 3.800 E-02 & 7.89 \\ \text { 6CU/F25 } & 1.170 E-02 & 5.13 \\ \text { GNB93/F25 } & 3.000 E-02 & 10.00 \\ \text { GAU197/F25 } & 1.000 E-01 & 2.00\end{array}$

$9.666 E-01$
$-1.132 E+06$
$6.613 E-03$
$5.812 E-09$
$2.303 E-05$

0.9666
1.0197
1.0253
1.0053

$1.003 E+00$

$-1.129 \mathrm{c}+06$

$6.555 \Sigma-03$

5. $8002-09$

2. $282 \mathrm{E}-05$

1.0028

1.0167

1.0163

0.9995

Centrol Reactivity Worths 15/kgi

$\begin{array}{lrr}H-1 & 3.692 E+02 & 12.50 \\ B E-9 & 3.086 E+00 & 14.89 \\ B-10 & -5.834 E+01 & 5.26 \\ C & 2.065 E+00 & 12.50 \\ \text { AL-27 } & 1.724 E-01 & 66.67 \\ F E & -2.776 E-02 & 200.00 \\ C 0-59 & -1.052 E-01 & 50.00 \\ N I & -7.924 E-01 & 6.67 \\ C U & -2.926 E-01 & 16.67 \\ A U-197 & -3.936 E-01 & 4.00 \\ T H-232 & -6.013 E-02 & 22.22 \\ U-235 & 6.662 E+00 & 0.99 \\ U-238 & 1.055 E+00 & 1.85 \\ P U-239 & 1.254 E+01 & 1.03 \\ P U-240 & 7.427 E+00 & 10.43\end{array}$

1.677E-01
$1.568 E+00$
8. $007 \mathrm{E}-01$
$1.391 \mathrm{0}+00$
$3.049 \mathrm{E}-03$
5. $923 \mathrm{E}-03$
8. $081 \mathrm{E}-03$
3. $003 \mathrm{E}-02$
8.672E-02

1.0181

0.9861

1.0522

0.9921

$\begin{array}{lll}.41 & 3.049 E-03 & 1.1292\end{array}$

5.13

2.00

0.1559

0.7591

1.0010

0.8672

$1.707 E-01$
$1.567 E+00$
$8.906 E-01$
$1.394 E+00$
$3.006 E-03$
$5.870 E-03$
$8.790 E-03$
$2.958 E-02$
$8.55 ! E-02$

1.0365

0.9857

1.0641

0.9943

1.1132

0.1515

0.7513

8. $672 \mathrm{E}-02$

0.9060

0.8551

$\begin{array}{rrrr}3.473 E+02 & 0.9400 & 3.638 E+02 & 0.9853 \\ 7.539 E+00 & 0.9323 & 7.960 E+00 & 0.9841 \\ -3.746 E+01 & 0.6366 & -4.910 E+01 & 0.8345 \\ 1.520 E+00 & 0.7362 & 1.099 E+00 & 0.5273 \\ 2.392 E-01 & 1.3874 & -5.683 E-02 & -0.3296 \\ 8.531 E-03 & -0.3073 & -2.376 E-01 & 0.5671 \\ 4.522 E-03 & -0.0430 & -2.351 E-01 & 2.2345 \\ -4.983 E-01 & 0.6289 & -9.071 E-01 & 1.1451 \\ -4.471 E-02 & 0.1527 & -3.553 E-01 & 1.2133 \\ -1.532 E-01 & 0.3893 & -4.077 E-01 & 1.0350 \\ 1.731 E-01 & -2.8793 & 3.066 E-03 & -0.0513 \\ 5.175 E+00 & 0.7768 & 6.527 E+00 & 0.9798 \\ 9.490 E-01 & 0.8996 & 1.056 E+00 & 1.0028 \\ 9.801 E+00 & 0.7816 & 1.253 E+01 & 0.9990 \\ 6.113 E+00 & 0.8231 & 7.777 E+00 & 1.0472\end{array}$

Goduvo Leakope Flux Compopinon

\begin{tabular}{|c|c|c|c|c|c|c|c|c|}
\hline $\begin{array}{r}\text { Fine } \\
\text { Groups }\end{array}$ & $\begin{array}{r}\text { Energy } \\
\text { Boundory } \\
\text { lev } \\
\end{array}$ & $\begin{array}{l}\text { Loth. } \\
\text { Wideh }\end{array}$ & $\begin{array}{c}\text { Moosured } \\
\text { flux } \\
\left(\mathrm{n} / \mathrm{cm}^{2} / \mathrm{s}\right)\end{array}$ & Uncer. & $\begin{array}{c}\text { Diffunion } \\
\text { Colculoted } \\
F l \text { ux } \\
\left.\text { (n } / \mathrm{cm}^{2} / \mathrm{s}\right)\end{array}$ & $C / E$ & $\begin{array}{l}\text { Tronome } \\
\text { alculoted } \\
\text { Flux } \\
\left(\mathrm{n} / \mathrm{cm}^{2} / \mathrm{s}\right)\end{array}$ & $C / E$ \\
\hline $\begin{array}{l}4-5 \\
6-7 \\
8-9 \\
10-11 \\
12-15 \\
16-19 \\
20-22 \\
23-24\end{array}$ & $\begin{array}{l}1.0000 E+07 \\
6.0653 E+06 \\
3.6780 E+06 \\
2.2313 E+06 \\
1.3533 E+06 \\
8.2085 E+05 \\
4.9787 E+05 \\
3.0197 E+05 \\
1.8316 E+05\end{array}$ & $\begin{array}{l}0.5 \\
0.5 \\
0.5 \\
0.5 \\
0.5 \\
0.5 \\
0.5 \\
0.5\end{array}$ & $\begin{array}{r}2.0 \\
7.8 \\
13.6 \\
16.8 \\
18.0 \\
17.5 \\
12.0 \\
7.2\end{array}$ & $\begin{array}{r}15.0 \\
5.1 \\
3.7 \\
3.6 \\
3.3 \\
4.0 \\
5.8 \\
9.7\end{array}$ & $\begin{array}{r}1.9232 \\
8.0060 \\
15.5820 \\
18.9112 \\
18.0000 \\
14.7528 \\
10.2845 \\
5.7404\end{array}$ & $\begin{array}{l}0.9516 \\
1.0264 \\
1.1457 \\
1.1257 \\
1.0000 \\
0.8430 \\
0.8570 \\
0.7973\end{array}$ & $\begin{array}{r}1.0255 \\
7.7217 \\
15.2334 \\
18.6916 \\
18.0000 \\
15.0717 \\
10.7501 \\
6.1052\end{array}$ & $\begin{array}{l}0.9127 \\
0.9900 \\
1.1201 \\
1.1126 \\
1.0000 \\
0.0512 \\
0.0558 \\
0.0401\end{array}$ \\
\hline
\end{tabular}


TABLE R-111

JEZEECL-23 RESULTS

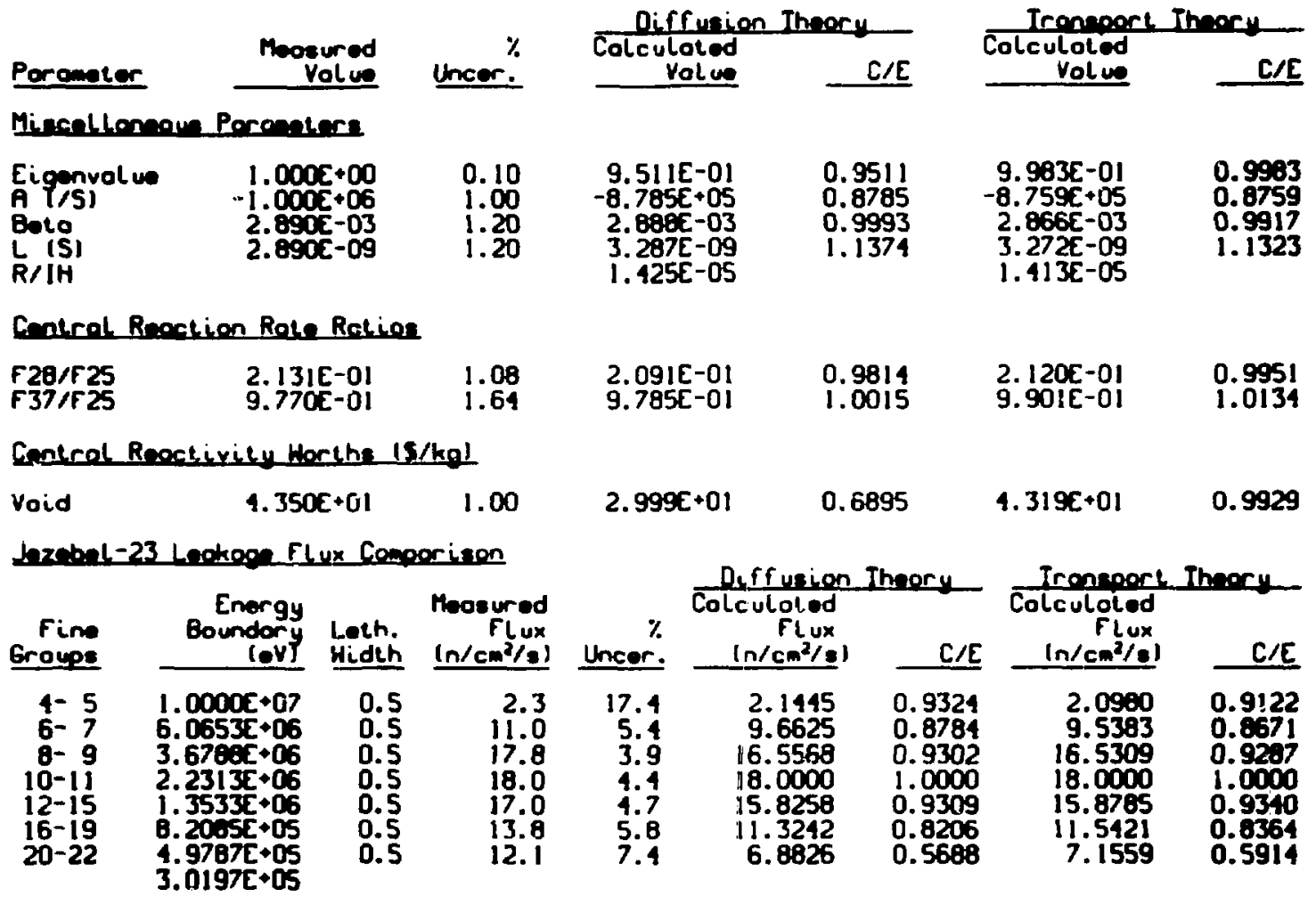


TAaLe A-IV

BIGTEN RESULTS

\begin{tabular}{|c|c|c|c|c|c|c|}
\hline \multirow[b]{2}{*}{ Porometer } & \multirow[b]{2}{*}{$\begin{array}{r}\text { Moosured } \\
\text { Volve } \\
\end{array}$} & \multirow[b]{2}{*}{$\begin{array}{r}\% \\
\text { Uncer. }\end{array}$} & \multicolumn{2}{|c|}{ Diffunion Theory } & \multicolumn{2}{|c|}{ Ironsport Theory } \\
\hline & & & $\begin{array}{r}\text { Colculated } \\
\text { Value }\end{array}$ & $\mathrm{C} / \mathrm{E}$ & $\begin{array}{r}\text { Colculoted } \\
\text { Volwe } \\
\end{array}$ & $\mathrm{C} / \mathrm{E}$ \\
\hline \multicolumn{7}{|c|}{ Gallhonenu Poroutere } \\
\hline $\begin{array}{l}\text { Eigenvol we } \\
\text { A (IS) } \\
\text { Boto } \\
L \text { (S) } \\
\text { R/IH }\end{array}$ & $\begin{array}{r}9.960 \varepsilon-01 \\
-1.170 z+05 \\
7.222 \varepsilon-03 \\
6.173 \varepsilon-00\end{array}$ & $\begin{array}{l}0.30 \\
0.85 \\
0.85 \\
0.85\end{array}$ & $\begin{array}{r}1.006 E+00 \\
-1.300 E+05 \\
7.215 E-03 \\
5.549 E-08 \\
2.040 E-05\end{array}$ & $\begin{array}{l}1.0096 \\
1.1113 \\
0.9990 \\
0.8989\end{array}$ & $\begin{array}{r}1.011 E+00 \\
-1.306 E+05 \\
7.202 E-03 \\
5.515 E-08 \\
2.037 E-05\end{array}$ & $\begin{array}{l}1.0147 \\
1.1161 \\
0.9972 \\
0.0934\end{array}$ \\
\hline
\end{tabular}

Centrel Reoction Role Rotios

\begin{tabular}{|c|c|c|c|c|c|c|}
\hline $\begin{array}{l}F 28 / F 25 \\
F 37 / F 25 \\
F 49 / F 25 \\
F 23 / F 25 \\
628 / F 25 \\
\text { ALI6/F25 } \\
\text { AB10/F25 } \\
\text { AFL } 27 / F 25 \\
\text { GCO5S/F25 } \\
\text { GCU/F25 } \\
\text { GAU197/F25 }\end{array}$ & $\begin{array}{l}3.730 E-02 \\
3.160 E-01 \\
1.165 E+00 \\
1.580 E+00 \\
1.100 E-01 \\
7.100 E-01 \\
1.011 E+00 \\
7.800 E-05 \\
9.500 E-03 \\
1.640 E-02 \\
1.670 E-01\end{array}$ & $\begin{array}{l}1.07 \\
1.58 \\
1.69 \\
1.90 \\
2.73 \\
1.41 \\
1.38 \\
2.56 \\
2.11 \\
6.10 \\
1.80\end{array}$ & $\begin{array}{l}1.007 E-02 \\
3.584 E-01 \\
1.192 E+00 \\
1.560 E+00 \\
1.078 E-01 \\
6.354 E-01 \\
8.531 E-01 \\
9.044 E-05 \\
9.065 E-03 \\
1.427 E-02 \\
1.536 E-01\end{array}$ & $\begin{array}{l}1.0742 \\
1.1341 \\
1.0059 \\
0.9872 \\
0.9797 \\
0.8950 \\
0.8438 \\
1.1595 \\
0.9542 \\
0.8704 \\
0.9198\end{array}$ & $\begin{array}{l}3.994 E-02 \\
3.578 \varepsilon-01 \\
1.192 E+00 \\
1.560 E+00 \\
1.078 \varepsilon-01 \\
6.357 E-01 \\
8.535 E-01 \\
9.028 E-05 \\
9.066 E-03 \\
1.428 \varepsilon-02 \\
1.537 E-01\end{array}$ & $\begin{array}{l}1.0706 \\
1.1322 \\
1.0057 \\
0.9872 \\
0.9600 \\
0.8951 \\
0.8442 \\
1.1574 \\
0.9545 \\
0.8707 \\
0.9202\end{array}$ \\
\hline
\end{tabular}

Centrol Reactivity Worths (S/kg)

\begin{tabular}{|c|c|c|c|c|c|c|}
\hline $\begin{array}{l}H-1 \\
L I-6 \\
L I-7 \\
B E-9 \\
B-10 \\
B-11 \\
C \\
0-16 \\
A L-27 \\
F E \\
P U-197 \\
T H-232 \\
U-233 \\
U-235 \\
U-238 \\
N P-237 \\
P U-238 \\
P U-239\end{array}$ & $\begin{array}{r}-1.789 E+01 \\
-1.291 E+01 \\
-2.768 E-01 \\
-2.555 E-01 \\
-1.024 E+01 \\
-2.394 E-01 \\
-1.674 E-01 \\
-9.452 E-02 \\
-6.991 E-02 \\
-6.482 E-02 \\
-1.246 E-01 \\
-8.966 E-02 \\
1.482 E+00 \\
8.497 E-01 \\
-4.262 E-02 \\
9.245 E-02 \\
9.148 E-01 \\
1.387 E+00\end{array}$ & $\begin{array}{l}15.38 \\
1.79 \\
14.29 \\
2.11 \\
0.41 \\
5.26 \\
2.75 \\
6.42 \\
3.68 \\
2.68 \\
1.13 \\
6.67 \\
0.80 \\
0.69 \\
0.95 \\
5.70 \\
2.55 \\
0.12\end{array}$ & $\begin{array}{r}-1.887 E+01 \\
-1.174 E+01 \\
-4.055 E-01 \\
-3.529 E-01 \\
-9.231 E+00 \\
-2.609 E-01 \\
-2.179 E-01 \\
-1.274 E-01 \\
-9.647 E-02 \\
-7.213 E-02 \\
-1.237 E-01 \\
-9.110 E-02 \\
1.500 E+00 \\
8.566 E-01 \\
-4.262 E-02 \\
1.583 E-01 \\
9.701 E-01 \\
1.396 E+00\end{array}$ & $\begin{array}{l}1.0547 \\
0.9096 \\
1.4651 \\
1.3812 \\
0.9015 \\
1.0900 \\
1.3019 \\
1.3475 \\
1.3800 \\
1.1128 \\
0.9931 \\
1.0160 \\
1.0123 \\
1.0081 \\
0.9954 \\
1.7118 \\
1.0605 \\
1.0064\end{array}$ & $\begin{array}{r}-1.956 E+01 \\
-1.186 E+01 \\
-4.163 E-01 \\
-3.681 E-01 \\
-9.317 E+00 \\
-2.698 E-01 \\
-2.257 E-01 \\
-1.326 E-01 \\
-9.670 E-02 \\
-7.320 E-02 \\
-1.253 E-01 \\
-9.235 E-02 \\
1.507 E+00 \\
8.599 E-01 \\
-4.3615-02 \\
1.565 E-01 \\
9.731 E-01 \\
1.102 E+00\end{array}$ & $\begin{array}{l}1.0935 \\
0.9186 \\
1.5112 \\
1.1409 \\
0.9099 \\
1.1270 \\
1.3482 \\
1.4030 \\
1.1117 \\
1.1292 \\
1.0054 \\
1.0301 \\
1.0168 \\
1.0120 \\
1.0191 \\
1.6929 \\
1.0637 \\
1.0108\end{array}$ \\
\hline
\end{tabular}

Bupten Lrokoen Flux Comporinon

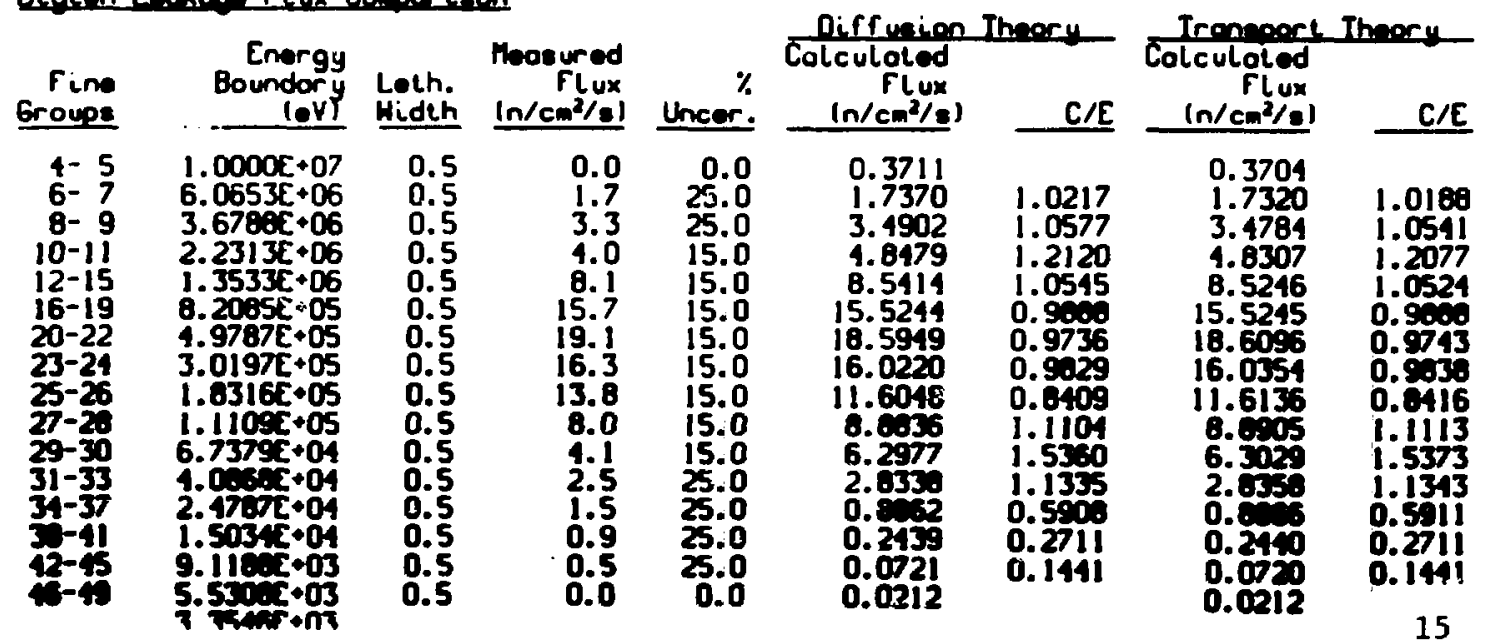


TFQLE $A-V$

JEZEBZ-PU RESULTS

\begin{tabular}{|c|c|}
\hline Porameter & $\begin{array}{l}\text { Moopured } \\
\text { Volue }\end{array}$ \\
\hline \multicolumn{2}{|c|}{ Miscollomene Poromters } \\
\hline $\begin{array}{l}\text { Eiganvolue } \\
\text { A }\{/ S \text { I } \\
\text { Beta } \\
L \text { (S) } \\
R / I H\end{array}$ & $1.000 E+00$ \\
\hline
\end{tabular}
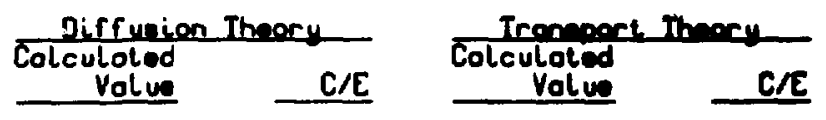

Central Reoction Rote Rotion

$\begin{array}{lllllll}\text { F28/F25 } & 2.060 E-01 & 1.46 & 1.869 E-01 & 0.9071 & 1.902 E-01 & 0.9231 \\ \text { F37/F25 } & 9.200 E-01 & 2.17 & 9.245 E-01 & 1.0049 & 9.358 E-01 & 1.0172\end{array}$

Contral Roativitu Horth Ration

$\begin{array}{lllllll}\text { PU238/U235 } & 2.010 E+\infty & 5.97 & 1.968 E+00 & 0.9791 & 2.006 E+00 & 0.9980 \\ \text { PU239/U235 } & 1.990 E+00 & 3.52 & 1.911 E+00 & 0.9619 & 1.951 E+00 & 0.9902 \\ \text { CH241/U235 } & 1.820 E+00 & 5.49 & 1.901 E+00 & 1.0446 & 1.951 E+00 & 1.0719\end{array}$

TFaLE A-YI

FLATTOP-25 RESULTS

\begin{tabular}{|c|c|c|c|c|c|c|}
\hline \multirow[b]{2}{*}{ Poromater } & \multirow[b]{2}{*}{$\begin{array}{l}\text { Meoeured } \\
\text { Volue }\end{array}$} & \multirow[b]{2}{*}{ Uncer. } & \multicolumn{2}{|c|}{ Diffurion Iheory } & \multicolumn{2}{|c|}{ Trongoast Thacy } \\
\hline & & & $\begin{array}{r}\text { Colculoted } \\
\text { Yolve }\end{array}$ & $\mathrm{C} / \mathrm{E}$ & $\begin{array}{r}\text { Colculoted } \\
\text { Volue }\end{array}$ & $\mathrm{c} / \mathrm{E}$ \\
\hline \multicolumn{7}{|c|}{ Misenthongun Promitere } \\
\hline $\begin{array}{l}\text { Eigenvol we } \\
\text { A }\{/ S \text { ) } \\
\text { Bolo } \\
L \text { (S) } \\
R / I H\end{array}$ & $\begin{array}{r}1.000 c+00 \\
-3.000+05 \\
6.650 c-03 \\
1.750 c-03\end{array}$ & $\begin{array}{l}0.10 \\
2.63 \\
2.63 \\
2.63\end{array}$ & $\begin{array}{r}9.144 E-01 \\
-2.712 E+05 \\
3.623 E-03 \\
1.336 E-08 \\
1.056 E-05\end{array}$ & $\begin{array}{l}0.9144 \\
0.7137 \\
0.5448 \\
0.7634\end{array}$ & $\begin{array}{r}1.015 E+00 \\
-4.216 E+05 \\
6.999 E-03 \\
1.659 E-08 \\
2.292 E-05\end{array}$ & $\begin{array}{l}1.0149 \\
1.1100 \\
1.0525 \\
0.9400\end{array}$ \\
\hline
\end{tabular}

\section{Centrol Recation lote Rotiven}

\begin{tabular}{|c|c|c|c|c|c|}
\hline $\begin{array}{l}F 23 / F 25 \\
F 20 / F 25 \\
F 37 / F 25 \\
F 49 / F 25\end{array}$ & $\begin{array}{l}\text { 1. } 600 z+00 \\
1.490 z-01 \\
7.600 z-01 \\
1.370 z+00\end{array}$ & $\begin{array}{l}1.88 \\
1.34 \\
1.32 \\
1.46\end{array}$ & $\begin{array}{l}1.570 E+00 \\
1.439 \varepsilon-01 \\
7.878 \varepsilon-01 \\
1.359 \varepsilon+00\end{array}$ & $\begin{array}{l}0.9813 \\
0.9660 \\
1.0366 \\
0.9919\end{array}$ & $\begin{array}{l}1.568 \mathrm{E}+00 \\
1.547 \mathrm{E}-01 \\
8.257 \mathrm{E}-01 \\
1.371 \mathrm{E}+00\end{array}$ \\
\hline
\end{tabular}

Central Reactivity Horthe 19/kgl

$\begin{array}{lll}U-233 & 1.497 E+01 & 1.29 \\ U-235 & 8.509 E+00 & 1.50 \\ U-230 & 1.00 E+00 & 3.51 \\ N-237 & 7.232 E+\infty & 1.75 \\ P U-230 & 1.421 E+01 & 1.33 \\ P U-239 & 1.591 E+01 & 0.79 \\ P U-242 & 7.455 E+\infty & 2.50 \\ P M-241 & 8.422 E+\infty 0 & 0.74\end{array}$

$1.749 \varepsilon+01$
$9.693 E+00$
$-8.846 E-01$
$6.126 \varepsilon+00$
$1.650 E+01$
$1.794 \varepsilon+01$
$6.190 \varepsilon+00$
$7.920 E+00$

1.1685

$1.3705+01$

$7.991 E+00$

$\begin{array}{rl}-0.8191 & 1.020 E+\infty 0 \\ 0.8065 & 7.757 E+\infty 0\end{array}$

$1.511 \mathrm{E}+01$

j. $5202+01$

1.1278

0.8304

$7.300 \tau+00$

$0.9404 \quad 9.157 t+00$

0.9151

0.9391

0.0515

1.0726

1.0030

0. 9005

0.072

1.0 .73 
TABLE R-VIJ

FLATTOP-PU RESULTS

\begin{tabular}{|c|c|c|c|c|c|c|}
\hline \multirow[b]{2}{*}{ Porometer } & \multirow[b]{2}{*}{$\begin{array}{l}\text { Moosured } \\
\text { Volve }\end{array}$} & \multirow[b]{2}{*}{$\begin{array}{r}\% \\
\text { Uncer. }\end{array}$} & \multicolumn{2}{|c|}{ Diffusion Theory } & \multicolumn{2}{|c|}{ Ironnoore Ireme } \\
\hline & & & $\begin{array}{r}\text { Colculated } \\
\text { Value } \\
\end{array}$ & $\mathrm{C} / \mathrm{E}$ & $\begin{array}{r}\text { Colculoted } \\
\text { Yolwe } \\
\end{array}$ & $C / E$ \\
\hline \multicolumn{7}{|c|}{ senllomous Poromaters } \\
\hline $\begin{array}{l}\text { genval ue } \\
? / S \text { ) } \\
\text { to } \\
\text { (S) } \\
\text { iH }\end{array}$ & $\begin{array}{r}1.000 E+00 \\
-2.140 E+05 \\
2.761 E-03 \\
1.290 E-08\end{array}$ & $\begin{array}{l}0.10 \\
2.34 \\
2.34 \\
2.34\end{array}$ & $\begin{array}{r}8.492 E-01 \\
-1.129 E+05 \\
1.020 E-03 \\
1.268 E-08 \\
1.297 E-05\end{array}$ & $\begin{array}{l}0.8492 \\
0.5277 \\
1.7457 \\
3.3085\end{array}$ & $\begin{array}{r}1.021 E+\infty 0 \\
-2.121 E+05 \\
2.819 \overline{0}-03 \\
1.329-0 . \\
9.270 E-06\end{array}$ & $\begin{array}{l}1.0207 \\
0.9910 \\
1.0210 \\
1.0302\end{array}$ \\
\hline
\end{tabular}

Central Reaction Rate Rotios

$\begin{array}{lllllll}F 28 / F 25 & 1.800 E-01 & 1.67 & 1.474 E-01 & 0.8189 & 1.693 E-01 & 0.9408 \\ F 37 / F 25 & 8.400 E-01 & 1.19 & 7.761 E-01 & 0.9239 & 8.516 E-01 & 1.0138\end{array}$

Centrol Reachivity Worthe $15 / \mathrm{kgl}$

$\begin{array}{lllllll}U-235 & 3.667 E+01 & 1.26 & 1.263 E+01 & 0.3445 & 3.469 E+01 & 0.9460 \\ U-238 & 2.206 E+00 & 2.76 & 1.024 E+00 & 0.4642 & 3.211 E+00 & 1.4554 \\ N P-237 & 3.392 E+01 & 1.35 & 1.043 E+01 & 0.3075 & 3.294 E+01 & 0.9712 \\ P U-238 & 6.223 E+01 & 1.47 & 2.176 E+01 & 0.3497 & 6.604 E+01 & 1.0513 \\ P U-239 & 6.846 E+01 & 0.44 & 2.277 E+01 & 0.3325 & 6.65 \%+01 & 0.9723 \\ P U-242 & 3.352 E+01 & 1.79 & 9.309 E+00 & 0.2926 & 3.124 E+01 & 0.9321 \\ A M-241 & 1.207 E+01 & 0.71 & 1.245 E+01 & 0.2959 & 4.0565+01 & 0.9642\end{array}$

TRQLE R-VIII

FLATTOP-23 RESLUTS

\begin{tabular}{|c|c|c|c|}
\hline & & & Diffusi \\
\hline Porameter & $\begin{array}{l}\text { Moosured } \\
\text { Volue }\end{array}$ & $\begin{array}{r}\% \\
\text { Uncer. }\end{array}$ & $\begin{array}{r}\text { Colculoled } \\
\text { Volue } \\
\end{array}$ \\
\hline
\end{tabular}

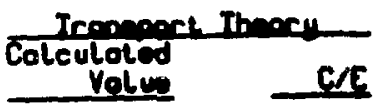

Miscellomens Poramere

\begin{tabular}{|c|c|c|c|}
\hline $\begin{array}{l}\text { Eigenvolus } \\
\text { A }(/ S) \\
\text { Botg } \\
L \text { (S) } \\
R / I H\end{array}$ & $\begin{array}{r}1.000 E+00 \\
-2.670 E+05 \\
3.645 E-03 \\
1.365 E-08\end{array}$ & $\begin{array}{l}0.10 \\
1.87 \\
1.87 \\
1.87\end{array}$ & $\begin{array}{r}8.451 E-01 \\
-2.170 E+05 \\
4.546 E-03 \\
2.095 E-08 \\
1.687 E-05\end{array}$ \\
\hline
\end{tabular}

Gentrol Reaction Rote Rotion

$\begin{array}{lllllll}\text { F28/F25 } & 1.910 E-01 & 1.57 & 1.661 E-01 & 0.8597 & 1.697 E-01 & 0.9030 \\ \text { F37/F25 } & 8.900 E-01 & 1.12 & 8.393 E-01 & 0.9431 & 9.153 E-01 & 1.0204\end{array}$

Central Rinctivity berthe 15/kol

$\begin{array}{lllllll}\text { Void } & 1.720 \varepsilon+01 & 0.74 & 3.145 E+01 & 0.6664 & 4.464 E+01 & 0.9457\end{array}$


Tface A-IX

\section{THCR RESULTS}
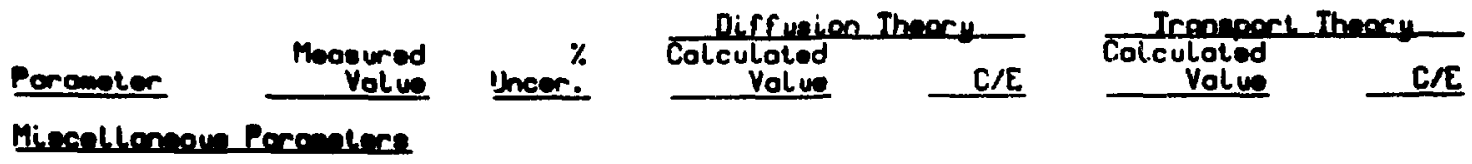

\begin{tabular}{|c|c|c|c|}
\hline $\begin{array}{l}\text { Eigenvolu } \\
\text { R }\{/ 51 \\
\text { Boto } \\
L \text { [S] } \\
R / I H\end{array}$ & $\begin{array}{r}1.000 c+00 \\
-1.970 c+05\end{array}$ & $\begin{array}{l}0.10 \\
5.08\end{array}$ & $\begin{array}{r}7.691 E-01 \\
-1.169 E+05 \\
2.533 E-03 \\
2.131 E-08 \\
9.567 E-06\end{array}$ \\
\hline
\end{tabular}

$\begin{array}{rr}0.7691 & 1.027 E+00 \\ 0.6036 & -1.746 E+05 \\ 2.116 E-03 \\ 1.212 E-08 \\ 8.233 E-06\end{array}$

1.0256

0.8661

Control Roction Rote Rotion

\begin{tabular}{|c|c|c|c|c|c|c|}
\hline $\begin{array}{l}F 20 / F 25 \\
F 37 / F 25 \\
F 02 / F 20 \\
629 / F 25 \\
2120 / 720 \\
602 / 620 \\
2102 / 2120\end{array}$ & $\begin{array}{l}1.950 z-01 \\
9.200 z-01 \\
2.600 z-01 \\
8.300 \tau-02 \\
5.300 c-02 \\
1.200 t+00 \\
1.010 c+00\end{array}$ & $\begin{array}{l}1.54 \\
2.17 \\
3.85 \\
3.61 \\
5.66 \\
5.00 \\
2.88\end{array}$ & $\begin{array}{l}1.648 E-01 \\
8.317 E-01 \\
2.458 E-01 \\
7.440 E-02 \\
5.532 E-02 \\
1.274 E+00 \\
1.013 E+00\end{array}$ & $\begin{array}{l}0.8451 \\
0.9040 \\
0.9453 \\
0.0964 \\
1.0437 \\
1.0616 \\
0.9736\end{array}$ & $\begin{array}{l}1.790 E-01 \\
8.854 \varepsilon-01 \\
2.473 \varepsilon-01 \\
7.090 \varepsilon-02 \\
6.407 \varepsilon-02 \\
1.282 \varepsilon+00 \\
1.02 z E+00\end{array}$ & $\begin{array}{l}0.9180 \\
0.9523 \\
0.9511 \\
0.8543\end{array}$ \\
\hline
\end{tabular}

Gentral Recativily inerthe 15/kgl

$\begin{array}{lllllll}\text { Void } & 6.641 E+01 & 1.94 & 6.118 E+01 & 0.9212 & 7.073 E+01 & 1.0651\end{array}$




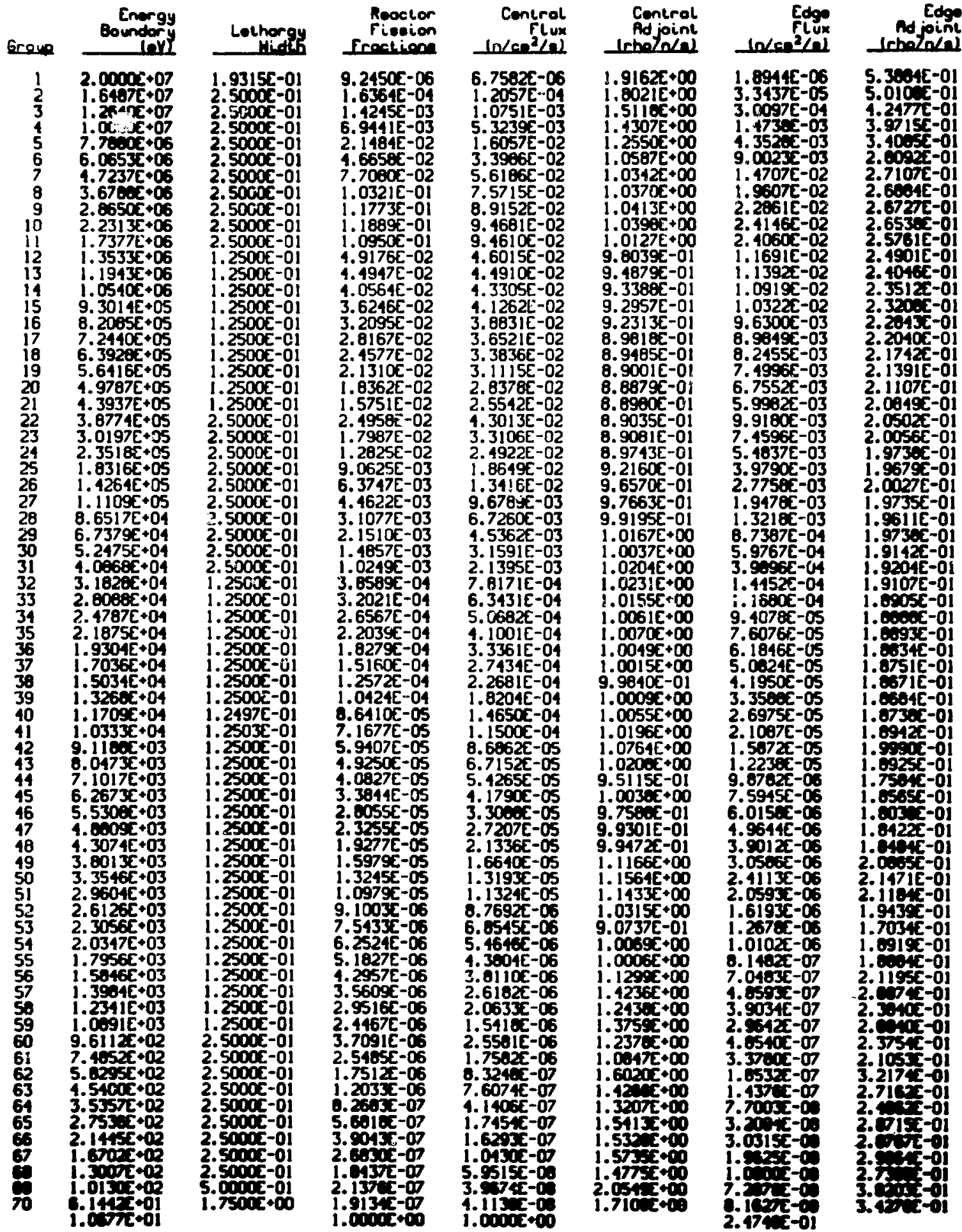


TFBLE A-XI

JEZEBEL 25-GROUP TRANSPORT THEORY SPECTRA

\begin{tabular}{|c|c|}
\hline Econe & $\begin{array}{r}\text { Energy } \\
\text { Boundory } \\
\text { Invil }\end{array}$ \\
\hline $\begin{array}{r}1 \\
2 \\
3 \\
4 \\
5 \\
6 \\
7 \\
6 \\
9 \\
10 \\
11 \\
12 \\
13 \\
14 \\
15 \\
16 \\
17 \\
18 \\
19 \\
20 \\
21 \\
22 \\
23 \\
24 \\
25\end{array}$ & 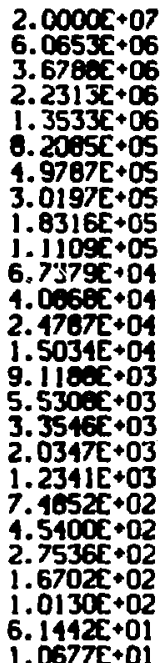 \\
\hline
\end{tabular}

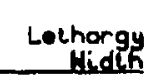

$1.1931 E+\infty 0$

5. $0000 \mathrm{c}-01$

5. $0000 \mathrm{E}-\mathrm{D}$

5. $0000 \mathrm{E}-01$

5. $0000 \mathrm{z}-01$

$5.0000 \mathrm{c}-01$

$5.0000 \mathrm{c}-01$

$5.0000 \mathrm{c}-01$

5. $0000 \mathrm{e}-01$

5. $0000 \mathrm{e}-01$

5. $0000 \mathrm{c}-01$

$5.0000 \mathrm{c}-01$

5. $0000 \mathrm{c}-01$

5. $0000 \mathrm{c}-01$

5. $0000 \mathrm{r}-01$

$5.0000 \mathrm{c}-01$

$5.0000 \mathrm{c}-01$

5. $0000 \mathrm{E}-01$

5. $0000 \mathrm{c}-01$

5. $0000 \mathrm{C}-01$

5. $0000 \mathrm{E}-01$

5. $0000 \mathrm{E}-01$

5. $0000 \mathrm{C}-01$

5. $0000 \mathrm{c}-01$

$1.7500 \mathrm{D}+00$
Rooctor

Fiseion Froctione

3.00265-02

1. $2374 E-01$

2. $2095 \mathrm{E}-01$

2. $28395-0$

$1.7093 \mathrm{E}-01$

$1.0615 E-01$

$5.907 z-02$

3. $0812 \Sigma-02$

$1.5437 E-02$

$7.5699 \mathrm{E}-03$

$3.6368 \varepsilon-03$

$1.7310 \mathrm{c}-03$

8. 2045E-04

3. $0004 E-04$

1.8333E-04

8. 6565E-05

4. $0067 \mathrm{E}-05$

1. $9292 \mathrm{E}-05$

9. $1074 E-06$

4. $2997 E-06$

2. $0301 E-06$

9.5861E-07

4. $52675=07$

2. $1378 \bar{E}-07$

$1.913\{E-07$

1.0000 +00

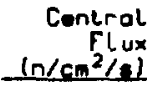

2. $2583 \mathrm{E}-02$

9. $0173 E-02$

1. 6487E-01

$1.8925 \mathrm{E}-01$
$1.7549 \mathrm{~T}-01$

1. $1030 \mathrm{E}-01$

$9.6933 E-02$

$5.8028 \mathrm{E}-02$

$3.2066 \bar{E}-02$

$1.6405 E-02$

$7.6954 E-03$

$3.5555 E-03$

$1.5248 E-03$

$6.7035 \mathrm{E}-04$

$2.5007 E-04$

$9.8272 E-05$

$4.0141 E-05$

$1.6274 E-05$

6. $1633 E-06$

2. 5907E-D6

1. 1748 -06

3. $374 \varepsilon \varepsilon-07$

1. $6382 z-07$

3.9674E-00

$1.113 \mathrm{cE}-08$

$1.0000 \mathrm{C}+00$
Central Ad joint Icholn/el

$2.8981 E+\infty 0$ $7.6626 \mathrm{E}-01$ $7.6091 \mathrm{E}-01$

$7.5|47 E-0|$ $1.3885 \mathrm{E}+00$ 1. $3203 E+\infty 0$ $9.7714 E-01$ 6.547|E-01 6. $9097 \varepsilon-01$ $7.2073 \mathrm{E}-01$ $7.3972 t-01$ $1.11995+\infty$ $1.4716 \overline{0}+00$ $1.4731 E+00$ $1.4836 \overline{0}+00$ $1.4936 c+00$ $1.5518 \mathrm{C}+00$ $1.66995+00$ $1.4123 \mathrm{E}+00$ 9.8363E-01 $1.00598+00$ $1.1255 E+\infty$ 1. $1170 \overline{0}+00$ $7.5234 \mathrm{E}-01$ 6. $26395-01$

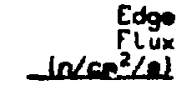

6. $16205-03$

2. $37095-12$

4. $2460 \mathrm{c}-02$

4.6206c-02

4. $4325 \bar{E}-02$

$3.4360 \mathrm{c}-02$

2. $2671 E-02$

1. $29435-02$

$6.7547 \varepsilon-03$

$3.26065-03$

1.4715E-03

$6.6028-04$

2. $3262 x-04$

$1.2360 \mathrm{c}-04$

4.5582E-05

$1.79102-05$

$7.357 \pi-06$

$3.0158 \mathrm{c}-06$

$1.172 x-06$

5. $0293 \mathrm{E}-07$

2. $2076 E-07$

6. $24095-00$

3. $0526 \mathrm{E}-00$

$7.2078 \mathrm{c}-09$

$0.1627 \mathrm{E}-09$

2. $4746 c-01$
Edge Ad joine (rholnol

8.0644E-01 $2.0209 x-01$ $1.9620-01$ $1.9140=01$ 3.5020 -01 3. $2224 \mathrm{E}-01$ 2. $2057 \varepsilon-01$ 1. $45805-01$ 1. $4537 t-01$ $1.4405 \mathrm{C}-01$ $1.4235 E-01$ $2.09405-01$ 2.7593c-01 2.7472:-01 $2.740 x-01$ $2.7755 \mathrm{E}-01$ 2. $0970 \mathrm{c}-01$ 3. $143 \%-0$ $2.725 x+01$ 1. 9rezt-01 i. some 01 2. $1045 E-01$ 2. 0ose-0i 1.3037E-01 $1.234-01$ 
There $n-x \mid 1$

coolve ro-gnow thms

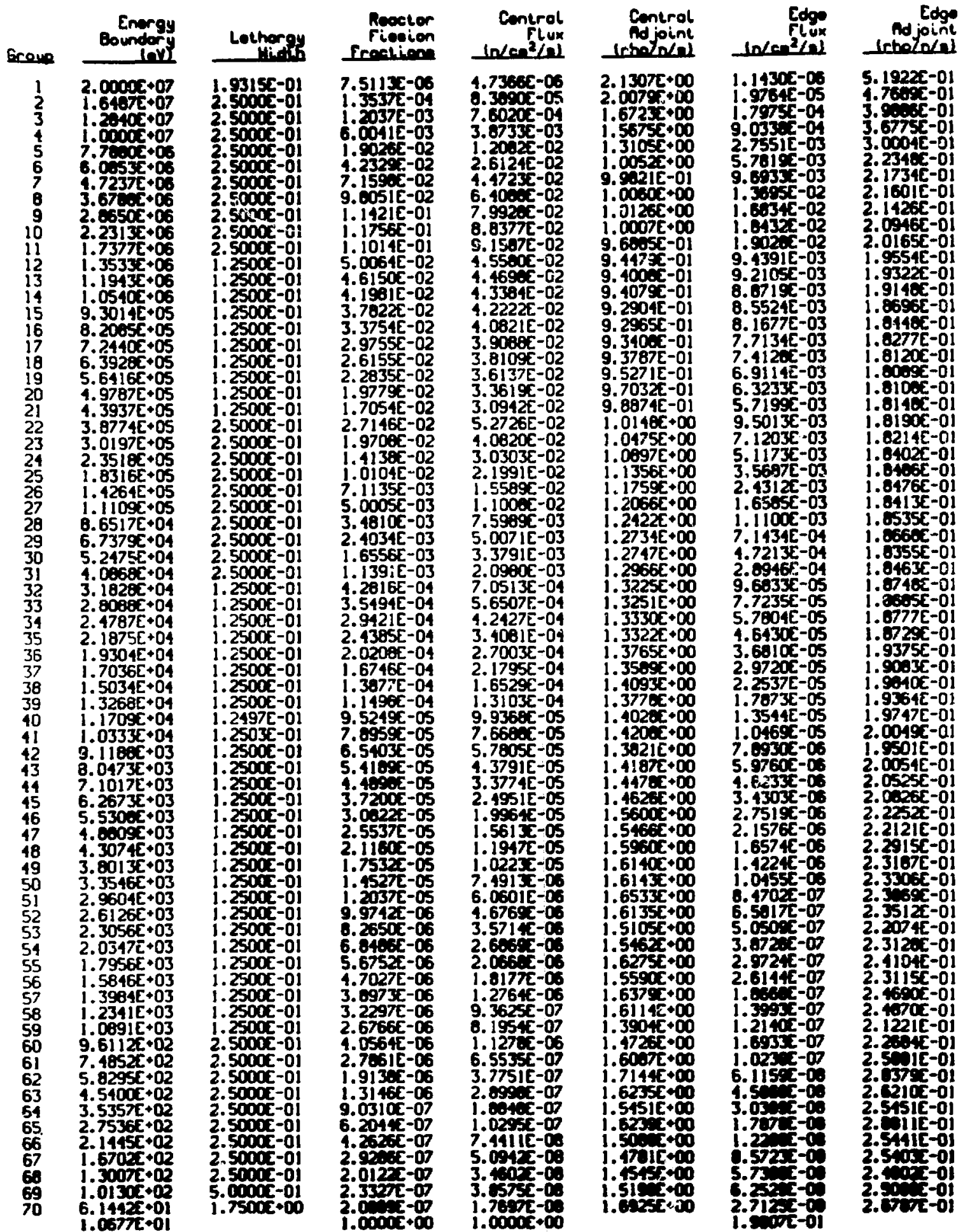


TABLE A-X 111

\begin{tabular}{c} 
\\
crome \\
\hline 1 \\
2 \\
3 \\
4 \\
5 \\
6 \\
7 \\
6 \\
9 \\
10 \\
11 \\
12 \\
13 \\
11 \\
15 \\
16 \\
17 \\
18 \\
19 \\
20 \\
21 \\
22 \\
23 \\
24 \\
25
\end{tabular}

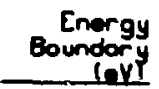

2. $0000 \mathrm{c}+07$

6. $0653 E+06$

$3.6700 \mathrm{C}+06$

2. $2313 \mathrm{E}+06$

$1.3533 E+06$

- $2005 E+05$

$4.9787 E+05$

$3.0197 E+05$

$1.0316 E+05$

$1.11095+05$

$6.7379 \mathrm{C}+04$

$4.0068 \mathrm{C} \times 4$

$2.1787 E+04$

$1.5034 E+04$

9. $1180 \mathrm{0}+03$

$5.5300 \overline{2}+03$

3. $3546 E+03$

2. $0347 \varepsilon+03$

$1.2341 E+03$

$7.40525+02$

$1.5400 \overline{0}+02$

$2.7536 E+02$

$1.670 x 2+02$

$1.0130 \mathrm{E}+02$

$6.1442 E+01$

$1.0677 E+01$

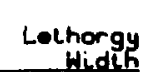

i. $1931 E+00$

5. $0000 \mathrm{E}-01$

5. $0000 \mathrm{E}-0$

$5.0000 \mathrm{E}-0$

5. $0000 \mathrm{E}-0$

5. $0000 \mathrm{E}-0$

5. $0000 \mathrm{E}-01$

5. $0000 \mathrm{E}-01$

5. $0000 \mathrm{E}-0$

$5.0000 \mathrm{E}-01$

5. $0000 \mathrm{c}-01$

$5.0000 \bar{c}-01$

5. $0000 \mathrm{e}-01$

5. $0000 \mathrm{r}-0$

5. $0000 \mathrm{E}-01$

5. $0000 \mathrm{E}-01$

5.0000 -01

5. $0000 \mathrm{z}-01$

5. $0000 \mathrm{E}-01$

5. $0000 \mathrm{E}-01$

5. $0000 \mathrm{c}-01$

5. $0000 \mathrm{c}-01$

5. $0000 \mathrm{c}-01$

5. $0000 \mathrm{c}-01$
GOOIVA 25-GROUP TRANSPORT THEORY SPECTRA

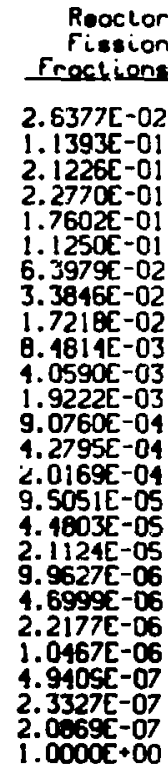

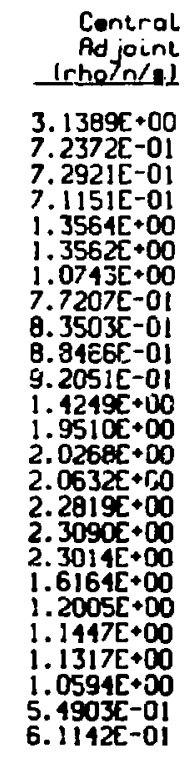

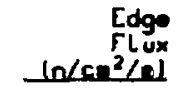

3. 859IE-03

1. $5475 E-02$

3. $0529 \mathrm{E}-02$

$3.746 \pi-02$

$3.6074 E-02$

$3.02055-02$

$2.1544 E-02$

$1.2236 \mathrm{C}-02$

5. $99995-03$

2.7685E-03

$1.1665 E-03$

1.6353E-04

$1.70765-04$

$6.4423 E-05$

2.1923E-05

$7.96945-06$

3.0557E-06

$1.13265-06$

$4.3067 \varepsilon-07$

1.6354E-07

7.6237 E-0.

$3.0166 E-00$

1. $4312 E-00$

$6.2529 \mathrm{C}-09$

2.7125E-09

$1.9007 \mathrm{E}-01$

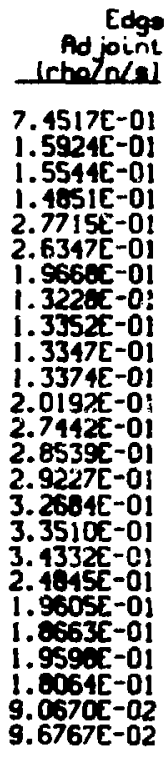

Edgo Irhothal

$150245-01$

$1.5544 E-01$

$1.4051 E-0$

$2.7715 \mathrm{E}-01$

1. $55605-0$

$1.335 x-0$

$1.3347 E-0$

.3374E-0

$2.74425-01$

539z-01

3. $26045-01$

3. $3510 \mathrm{E}-0$

$2.1045 \mathrm{E}-0$

s:-0

$1.95905-0$

$90705-02$

9.6767t-02 
THOLE A-XIV

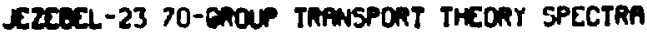

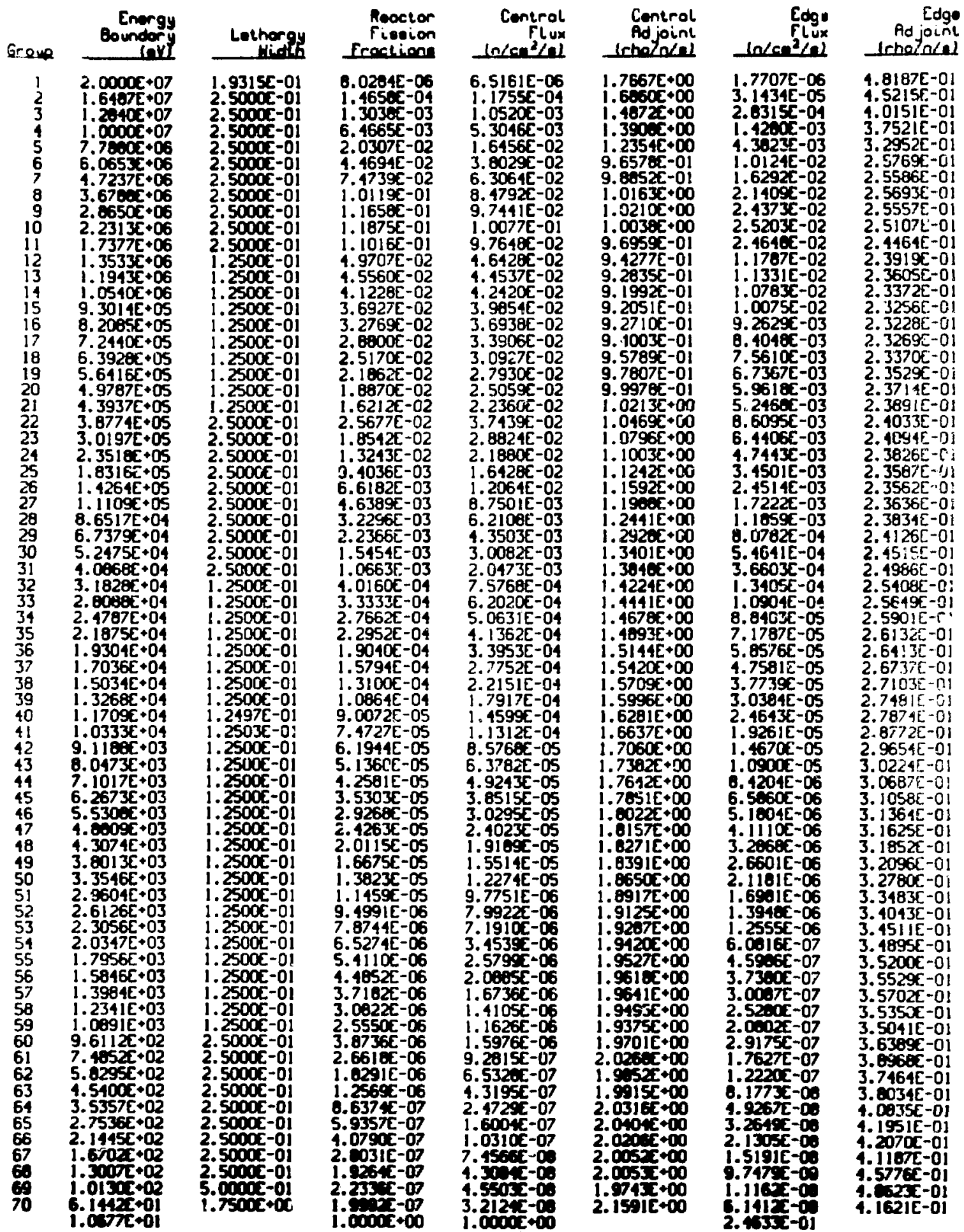


IFOLE A-XYI

DIGTEN 70-GROUP TAANSPORI IHCONY SPECTRA

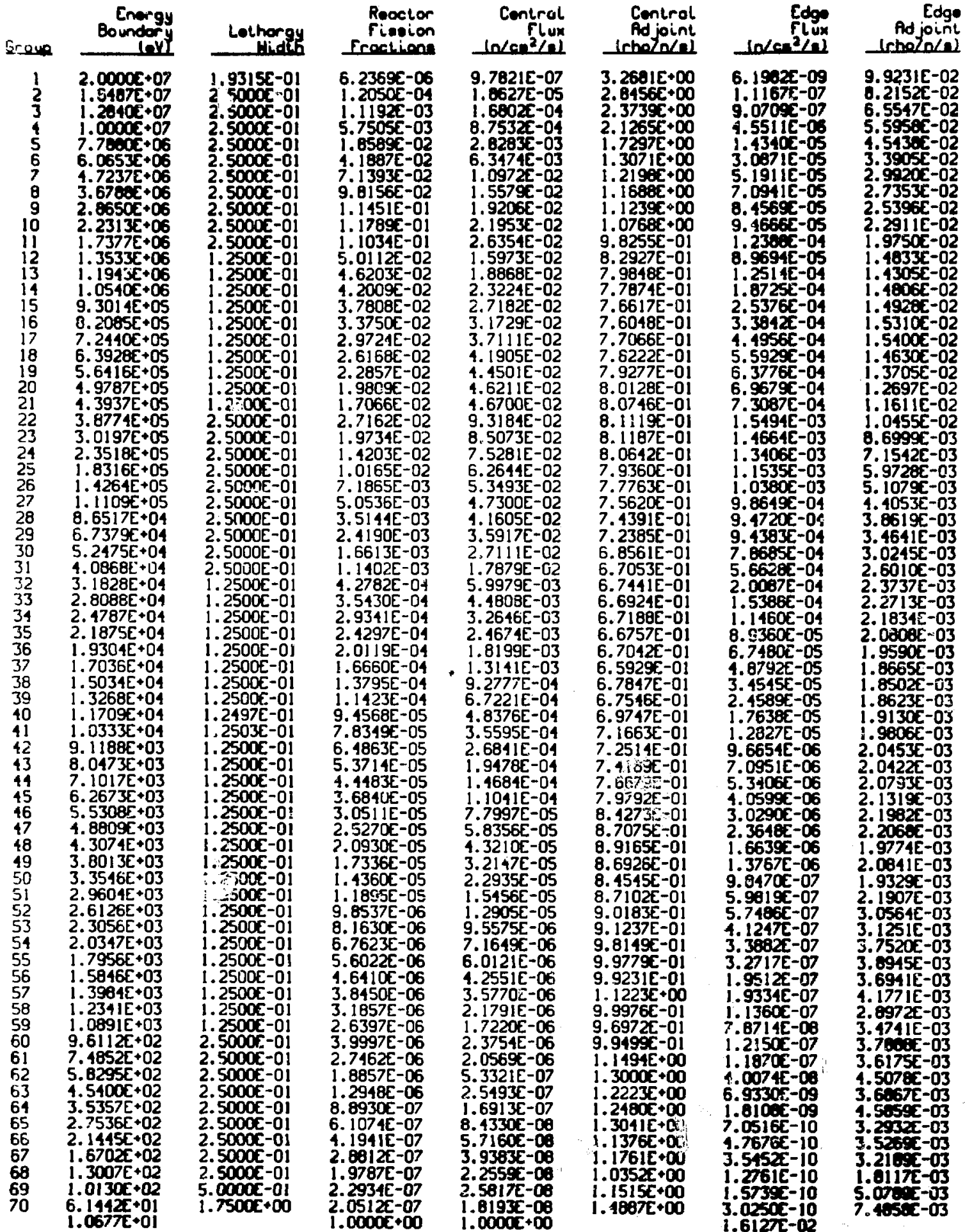


Trace $n-x y I I$

BIGTEN 70-6mour zane Fission ramctions

\begin{tabular}{|c|c|c|c|c|}
\hline ang & $\begin{array}{r}\text { Energy } \\
\text { Doundery } \\
\text { (nvil }\end{array}$ & $\begin{array}{l}\text { Lothorgy } \\
\text { Hindth }\end{array}$ & Corn & Anflectex \\
\hline $\begin{array}{l}1 \\
2 \\
3 \\
4 \\
5 \\
6 \\
7 \\
8 \\
9 \\
10 \\
11 \\
12 \\
13 \\
14 \\
15 \\
16 \\
17 \\
18 \\
19 \\
20 \\
21 \\
22 \\
23 \\
24 \\
25 \\
26 \\
27 \\
28 \\
29 \\
30 \\
31 \\
32 \\
33 \\
34 \\
35 \\
36 \\
37 \\
38 \\
39 \\
40 \\
41 \\
42 \\
43 \\
44 \\
45 \\
46 \\
47 \\
48 \\
49 \\
50 \\
51 \\
52 \\
53 \\
54 \\
55 \\
56 \\
57 \\
58 \\
59 \\
60 \\
61 \\
62 \\
63 \\
64 \\
65 \\
66 \\
67 \\
60 \\
69 \\
70\end{array}$ & 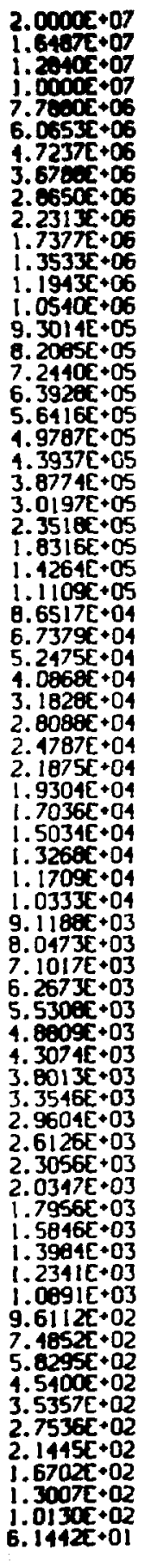 & 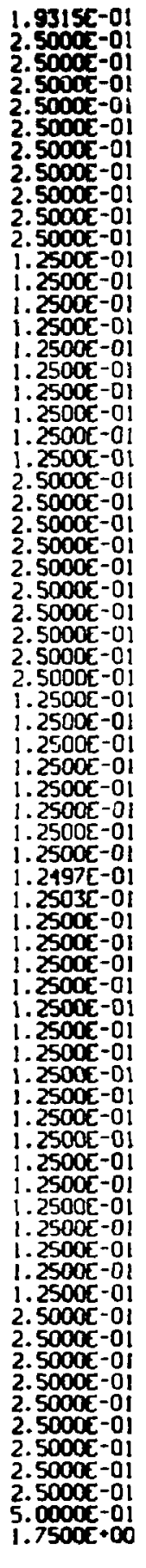 & 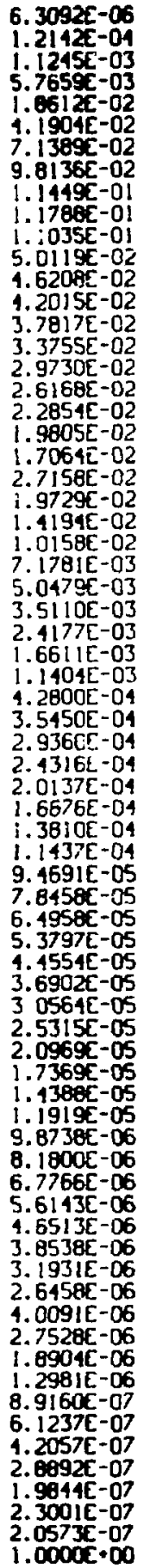 & $\begin{array}{l}5.0669 E-06 \\
1.0561 E-04 \\
1.0330 E-03 \\
5.5014 E-03 \\
1.8207 E-02 \\
4.1615 E-02 \\
7.1451 E-02 \\
9.8465 E-02 \\
1.1466 E-01 \\
1.1806 E-01 \\
1.1024 E-01 \\
4.9967 E-02 \\
4.6121 E-02 \\
4.1916 E-02 \\
3.7667 E-02 \\
3.3660 E-02 \\
2.9626 E-02 \\
2.6174 E-02 \\
2.2892 E-02 \\
1.9876 E-02 \\
1.7106 E-02 \\
2.7233 E-02 \\
1.9816 E-02 \\
1.4348 E-02 \\
1.0282 E-02 \\
7.3229 C-03 \\
5.1460 E-03 \\
3.5684 E-03 \\
2.4407 E-03 \\
1.6659 E-03 \\
1.1371 E-03 \\
4.2503 E-04 \\
3.5119 E-04 \\
2.9021 E-04 \\
2.3984 E-04 \\
1.9822 E-04 \\
1.6385 E-04 \\
1.3545 E-04 \\
1.1198 E-04 \\
9.2570 E-05 \\
7.6587 E-05 \\
6.3321 E-05 \\
5.2373 E-05 \\
4.3321 E-05 \\
3.5639 E-05 \\
2.9651 E-05 \\
2.4534 E-05 \\
2.0302 E-05 \\
1.6802 E-05 \\
1.3906 E-05 \\
1.1510 E-05 \\
9.5283 E-05 \\
7.8881 E-06 \\
6.5300 E-05 \\
5.4055 E-06 \\
4.4749 E-06 \\
3.7040 E-06 \\
3.0673 E-06 \\
2.5404 E-06 \\
3.8477 E-06 \\
2.6392 E-06 \\
1.8102 E-06 \\
1.2412 E-06 \\
8.5203 E-07 \\
5.8436 E-07 \\
4.0056 E-07 \\
2.7507 E-07 \\
1.8059 E-07 \\
2.1850 E-07 \\
1.9520 E-07 \\
1.0000 E+00\end{array}$ \\
\hline
\end{tabular}


Thate A-xy III

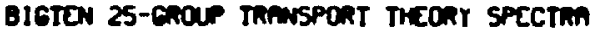

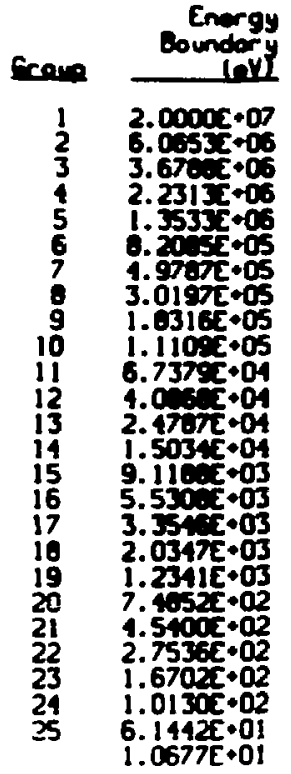

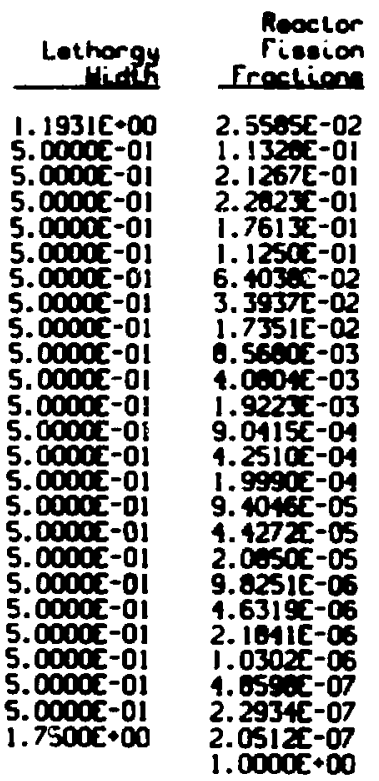

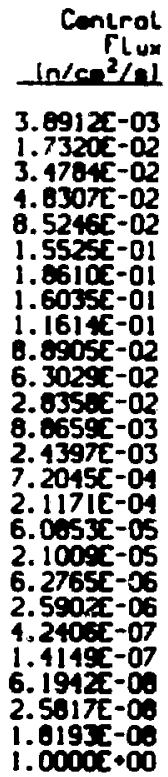

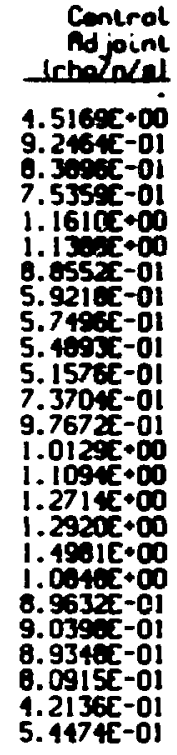

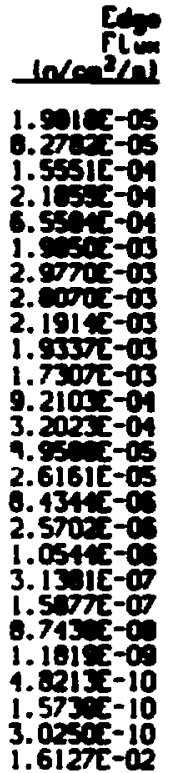

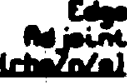

$1.2725-01$ 2. $\sin =-\frac{10}{40}$ i. गII -6 2. ISA: -02 2. 1 1. 2दार-क 5. ol $=-\mathrm{cos}$ 4. C. $15=0$ 3.1. $2.3 \times 1-0$ 2. 3 if $=-\infty$ 2. is -03 2. $\pi 3.5-03$ 3.0. $01-03$ 3.0. $3.776-03$ S. $7.1-13$ $3.71 x-01$ 2. 735:-01 $3.027 \div-03$ 2. $1=2-03$ 1. $19=03$ $1.0-2-03$ $2.73,2-03$

Tfrese $A-x \mid x$

BIGTEN 25-GROUP ZONE FISSION FRACTIONS

\begin{tabular}{|c|c|c|c|c|}
\hline 04 & $\begin{array}{r}\text { Energy } \\
\text { Boundory } \\
\text { InVI }\end{array}$ & $\begin{array}{c}\text { Lethor } \\
\text { Hit }\end{array}$ & Core & RAfl \\
\hline $\begin{array}{r}1 \\
2 \\
3 \\
4 \\
5 \\
6 \\
7 \\
8 \\
9 \\
10 \\
11 \\
12 \\
13 \\
14 \\
15 \\
16 \\
17 \\
18 \\
19 \\
20 \\
21 \\
22 \\
23 \\
24 \\
25\end{array}$ & 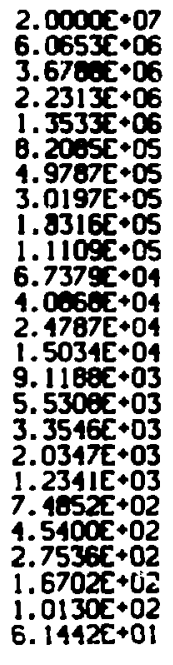 & 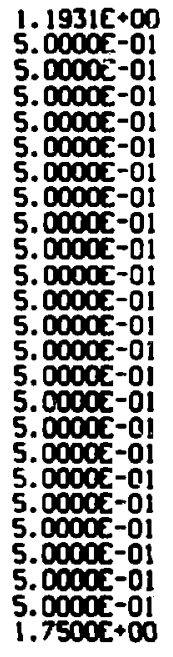 & 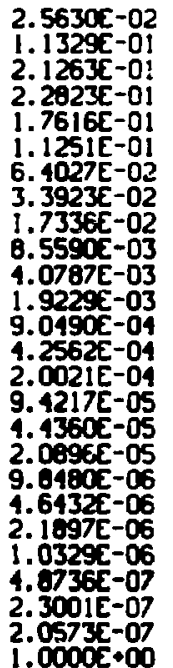 & 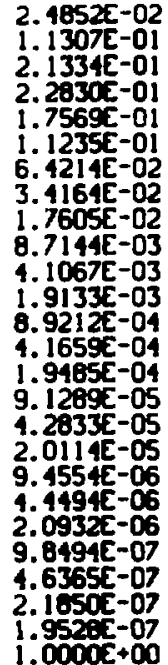 \\
\hline
\end{tabular}




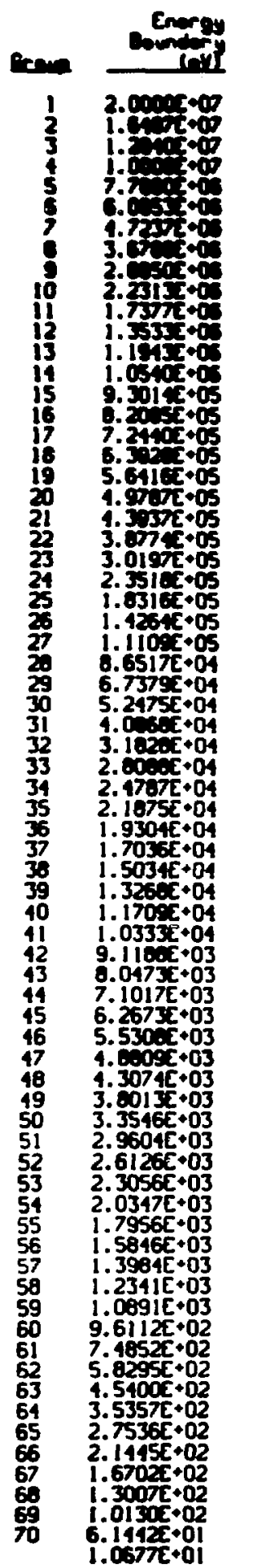

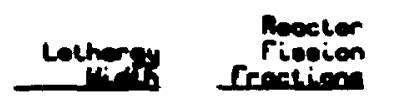

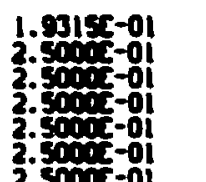

2. $31,-0$

2.

2. $5002-01$

2. $5001-0$

1. 2 roc-01

1. $200-0$

$1.2500 \mathrm{~L}-0$

1. $2500 \mathrm{c}-01$

$1.2500 \mathrm{C}-01$

$1.2500-01$

1. $2500 \mathrm{c}-0$

1. $2500 \mathrm{C}-01$

1. $25002-01$

1. $2500 \mathrm{C}-01$

2. $5000 \mathrm{~T}$ - 01

2. $5000 \mathrm{x}-01$

2. $5000 \mathrm{x}-01$

2. $5000 \mathrm{t}-01$

$2.5000 \mathrm{c}-01$

2. $5000 \mathrm{~L}-01$

2. $5000[-0$

2. $5000 \bar{c}-01$

2. $5000 \mathrm{c}-01$

2.5000c-01

$1.2500 \mathrm{c}-01$

1. 25005-01

$1.2500 \mathrm{C}-01$

1. $2500 \mathrm{C}-01$

1. $2500 \mathrm{c}-01$

1. $25005-01$

1. $25005-01$

1. $2500 \mathrm{c}-01$

$1.2497 \mathrm{E}-01$

$1.2503 E-01$

1. $2500 \mathrm{c}-0$

$1.2500 \mathrm{C}-01$

1. $2500 \mathrm{E}-01$

$1.25005-01$

1. $2500 \mathrm{c}-0$ 1

$1.2500 \mathrm{C}-01$

1. $2500 \mathrm{C}-01$

1. $25005-01$

$1.2500 \mathrm{C}-01$

1. $2500 \mathrm{E}-01$

$1.25002-01$

1. $2500 \mathrm{c}-01$

$1.2500 \mathrm{c}-01$

$1.2500 \mathrm{c}-01$

1. $2500 \mathrm{C}-01$

$1.2500 \mathrm{c}-01$

1. 2500c-01

1. $2500 \mathrm{E}-01$

2. 5000E-01

2. 5000E-01

2. $5000 \bar{E}-01$

2. $5000 \mathrm{C}-01$

2. $5000 \mathrm{c}-01$

2. $5000 \mathrm{C}-01$

2. $5000 \mathrm{c}-01$

2. $5000 \mathrm{C}-01$

2. $5000 \mathrm{c}-01$

5. 0000 -01
1. 1937z-0s

1. $57-2-04$

1.50 ce-03

.0.2-03

2. 154:-02

$1.6355=02$

$7.620+5-02$

$1.021 \alpha-01$

$1.1671 \mathrm{E}-01$

$1.1021 E-01$

$1.0927 \mathrm{c}-01$

$4.92135-02$

4.507 IE-02

4.0749-02

$3.6476-02$

. 2354-02

2. $8410 \mathrm{10}-02$

$2.4853 c-02$

2. 1576z-02

$1.851 .5 E-02$

$1.59155-02$

2.536: $\tau-02$

$1.831 \% 5-02$

1. उसO1E-02

9. $2526 E-03$

$6.513 t E-03$

4.562IE-03

3. $17905-03$

2.2010c-03

$1.5205 E-03$

1. $0491 \mathrm{E}-03$

3.950iE-04

3. 2780E-04

$2.7190 \mathrm{C}-04$

$2.2563 E-04$

$1.8715 E-04$

$1.5521 E-04$

$1.2872 E-04$

$1.0673 \mathrm{E}-04$

8.8478 $\mathrm{E}-05$

$7.3393 E-05$

6.0831E-05

$5.0430 \mathrm{t}-05$

4. $1806 E-05$

3. $46565-05$

2.8728c-05

2. $381 \mathrm{t}-05$

$1.9740 \mathrm{E}-05$

$1.63625-05$

$1.3563 E-05$

1. $1242 E-05$

9. $3100 \mathrm{c}-05$

$7.7244 E-06$

6. 4026E-06

5. $3071 E-06$

4. $3909 \varepsilon-06$

$3.6464 E-06$

3. 0225E-06

2. $5054 \mathrm{E}-0 \mathrm{~S}$

3. $7982 E-06$

2.6097E-06

$1.7932 E-06$

1.2322E-06

8. $46695-07$

5.8183E-07

3. $993 x-07$

2.7475t-07

1. 8881E-07

2. $1893 x-07$

1. $96022-07$

$1.0000 \mathrm{C}+00$
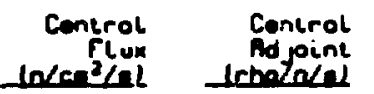

6. 5220:-06

1. $33745-04$

1. 110T-03

5.2055e-03

$1.55725-02$

$3.29555-02$

$5.4231 E-02$

$7.30205-02$

$0.6330 \mathrm{c}-02$

9. $2145 t-02$

9. $2900 \mathrm{t}-02$

$4.5637 \mathrm{E}-02$

1. 4752E-02

4. $3375 \mathrm{E}-02$

4. $1580 \mathrm{c}-02$

3.941 x-02

$3.7222 t-02$

3.4637 -02

3. $1993 \mathrm{E}-02$

2. $9321 \mathrm{E}-02$

2. $6547 \mathrm{E}-02$

4. $4900 \mathrm{E}-02$

$3.4713 k-02$

$2.6197 \mathrm{E}-02$

1. $9625 \mathrm{E}-02$

$1.415 x-02$

$1.0226 \varepsilon-02$

7. 1496E-03

1.8610 -03

3. $3668 €-03$

2. $2823 E-03$

6.3143E-04

$6.7326 \mathrm{E}-04$

$5.3740 t-04$

4. $3333 \mathrm{E}-\mathrm{O}$

$3.5161 \%-04$

2. $8813 \mathrm{E}-04$

2. $3763 \mathrm{E}-04$

1. $900 \div 5-04$

1. 5248E-04

1. $1950 E-04$

9. $0539 \mathrm{r}-05$

7.0060E-05

5.6436c-05

4. 3767E-05

3. $4724 E-05$

2. $8358 \mathrm{C}-05$

$2.2300 \varepsilon-05$

1.7499 -05

1. 3701E-05

i. $1948 \mathrm{E}-05$

9.2241E-06

7.1554E-06

5. 6903K-06

4.5617E-DG

3. $9212 E-06$

2.74298-06

2. $1445 \varepsilon-06$

1.6374E-06

2.6187E-06

1.8493E-06

8.6832E-07

7.4767E-07

4.0306E-07

$1.8505 E-07$

1. $66135-07$

1.0615E-07

6. 2525E-08

1. $1924 E-08$

$4.4482 E-08$

$1.0000 \mathrm{E}+00$
Irbotal

$1.960 \% 5 \cdot 00$

$1.6463 \times 00$

1.5654 - 00

$1.47772+00$

$1.2010 .+00$

1.0694E+00

$1.0464 E+00$

$1.0512 x+00$

$1.0546 E+00$

$1.0527 E+00$

$1.0222 E+00$

9. 89965-01

9. $6302 \mathcal{E}-01$

9. $1743 E-01$

9. $3331 \mathrm{E}-01$

9. $1303 x-01$

8. $7992 E-0$

8. 6523t-01

8. 4846ᄃ -01

8. $3733 \mathrm{E}-01$

8. $3333 E-01$

B. 3273E-01

8. 3354E-01

8. $4067 E-0$

8.6264E-01

9. 0233E-01

9. $1403 E-01$

9. $3004 E-01$

$9.5361 \mathrm{~L}-0$

$9.42 ! 4 E-0 !$

$9.5818 E-0$

$9.6172[-01$

$9.5588 E-01$

$9.5020 E-0$ !

$9.5198 E-01$

9.5086E-0]

9. $4863 E-0$ !

9. $1701 E-01$

9. 4995E-0!

9. $5440 \Sigma-0$

9.6812E-01

$1.0207 E+00$

9.7387E-0

9. i 705e-0i

9. $6728 E-0$

$9.4656 \mathrm{E}-01$

$9.64195-0$

$9.6727 \mathrm{E}-0$ !

$1.0744 E+00$

$1.1092 E+00$

i. $1114 E+00$

1. $00065+00$

8.9764E-01

9. $9684 E-01$

$9.83925-01$

$1.1048 E+00$

$1.3593 \varepsilon+00$

$1.19726+00$

$1.3341 \mathrm{E}+00$

1. $1878 \mathrm{E}+00$

$1.0742 x+00$

$1.5406 E+00$

$1.3807 \mathrm{E}+00$

$1.2776 E+00$

$1.52665+00$

$1.5321 \mathrm{E}+00$

$1.5345 E+00$

$1.4526 E+00$

$1.9029 \mathrm{C}+00$

$1.7094 \mathrm{E}+00$

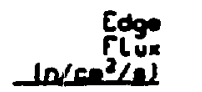

2. 3601E-06

$3.672 x-05$

3. $0705 x-0$

$1.442 \pi-03$

4.154ce-03 
The A-XXI

\begin{tabular}{|c|c|}
\hline ing & $\begin{array}{r}\text { Enorgy } \\
\text { Boundory } \\
\text { (AVI }\end{array}$ \\
\hline $\begin{array}{l}1 \\
2 \\
3 \\
4 \\
5 \\
6 \\
7 \\
8 \\
9 \\
10 \\
11 \\
12 \\
13 \\
14 \\
15 \\
16 \\
17 \\
10 \\
19 \\
20 \\
21 \\
22 \\
23 \\
24 \\
25\end{array}$ & 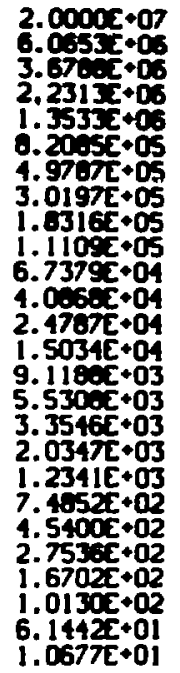 \\
\hline
\end{tabular}

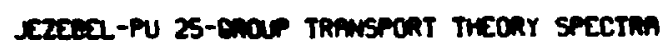

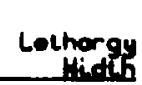

$1.1931 E+\infty 0$

5. $0000 \mathrm{c}-01$

5. $0000 \mathrm{c}-01$

$5.0000 \mathrm{t}-01$

5. $0000 \mathrm{c}-01$

5. $0000 \mathrm{t}-0$

5. Orope-01

5. $0000 \mathrm{E}-0$

5. $0000 \mathrm{c}-01$

5. $0000 \mathrm{c}-01$

5. $0000 \mathrm{c}-01$

5. $0000 \mathrm{~T}-01$

5. $0000 \mathrm{c}-01$

5. $0000 \mathrm{c}-01$

5. $0000 \mathrm{c}-01$

5. $0000 \mathrm{c}-01$

5. $0000 \mathrm{t}-01$

5. $0000 \mathrm{c}-01$

5. $0000 \mathrm{c}-01$

5. 0000 - 01

5. $0000 \mathrm{c}-01$

5.0000 -0

$5.0000 \bar{\varepsilon}-01$

5. $0000 \mathrm{c}-01$

$1.75000+\infty 0$
Reoctor

fiseion Iroction

3. 0356ع-02

1. 2264 4 -01

2.1005ร-01

$2.2749 E-01$

1.715iE-01

$1.07235-01$

5.9970 -02

3. $1397-02$

$1.5766 \mathrm{c}-02$

$7.7411 E-03$

$3.7215 \mathrm{E}-03$

$1.7719 z-03$

8. 3997E-04

3. $9732 x-04$

1.8772E-04

8. $6543 \mathrm{c}-05$

4. $1846 \mathrm{C}-05$

1.975sE-05

9. $3261 E-06$

4. $4030 \mathrm{C}-06$

2. $07095-06$

9. 81645-07

4. $6356 \mathrm{E}-07$

2. $10935-07$

$1.96025-07$

1.0000 . 00

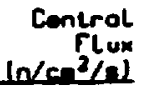

$2.21108-02$

$8.7184 c-02$

1.5935E-01

$1.6513 \mathrm{E}-01$

$1.43265-01$

$1.0078 \mathrm{c}-01$

6. $0910 \mathrm{c}-02$

$3.3777 \mathrm{E}-02$

$1.7376 E-02$

6. $247 \varepsilon \bar{c}-03$

$3.7870 \mathrm{E}-03$

$1.6105 \mathrm{E}-03$

$6.9965 \mathrm{E}-04$

$2.6000 \mathrm{E}-04$

$1.02606-04$

4. $2026 \bar{c}-05$

$1.6916 \varepsilon-05$

$6.1005 E-06$

$2.7176 E-06$

$1.1507 E-06$

$3.5117 \pi-07$

1.6867E-07

4. $1924 \varepsilon-\infty$

4. $446 x-00$

1. $0000 \mathrm{x}+\infty$
Central Ad joint Gholocal

$2.9727 E+00$

$7.7345 E-01$

$7.6977 \mathrm{E}-01$

$7.5047 \mathrm{E}-01$

$1.40145+00$

$1.2016 c+\infty$

9.15i IE-0I

6. $1200 E-01$

$6.45182-01$

$6.74095-01$

6. $9290 \mathrm{c}-01$

1.0512500

$1.3997 \mathrm{E}+00$

i. $396 x+00$

$1.41795+\infty$

$1.4440+\infty$

$1.5057 \varepsilon+\infty$

$1.6250 \Sigma+00$

i. $3595 \mathrm{E}+00$

9.5506 -01

9.7176E-01

$1.1181 \mathrm{C}+\infty$

$1.0919=+\infty$

$7.2486 \bar{E}-01$

$6.2485 t-01$
Edge
$\ln x=2 / x \mid$

5. $943 x-03$

$2.2005-02$

1.0535 -02

1. $65302-02$

1. $3.2,2-02$

3. $1573-02$

2. $310-0$

1. $33202-02$

6. $9700 \mathrm{z}-03$

$3.30 .032-03$

$1.53752-03$

6. $9430-04$

2. $900-2-04$

1.2520:-01

$1.6200 \mathrm{c}-05$

$1.824 \pi-05$

$7.47955-0$.

$3.0491 \mathrm{E}-\mathrm{\alpha}$

$1.10355-0$.

5. $1231 E-07$

2. $00645-07$

$6.3636 \mathrm{c}-0$

3. 0001E-0.

7.5757t-00

B.6273z-09

$2.43455-01$
Edo

Ad joint Intotal

- 172= $5-01$

$2.0170 \mathrm{C}-01$

1. $125-01$

i.sort-01

3. $25=01$

3. cosst -01

2. $1005-01$

1. $334 \%-01$

i. 32.5-01

i. 3100-0i

$1.30252-01$

i.917T-01

2.53465-01

$2.53142-01$

$2.5545 \mathrm{C}-01$

2. $\cos 5-01$

2. $73052-01$

2. $9700 \mathrm{C}-01$

2.5495-01

$1.8553 x-01$

$1.70302-01$

2.0945-01

2. $0000-01$

$1.32545-0$

$1.2234 \mathrm{E}-01$ 
Trace A-xxII

PLATTO-25 70-chour twhwspont theony SPECTRA

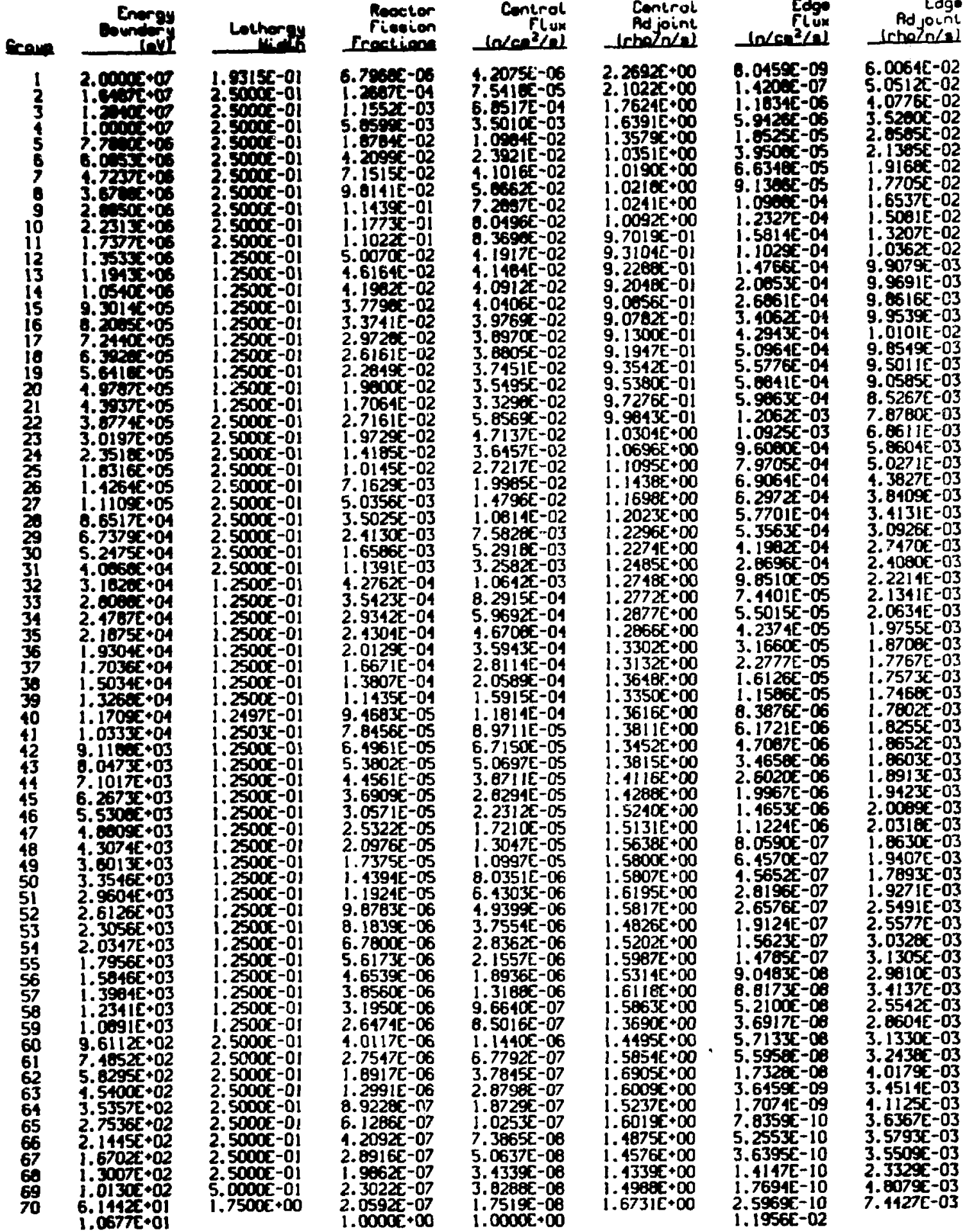


The A-XXIII

FLATto-25 70-empe 201: Fission rmotions

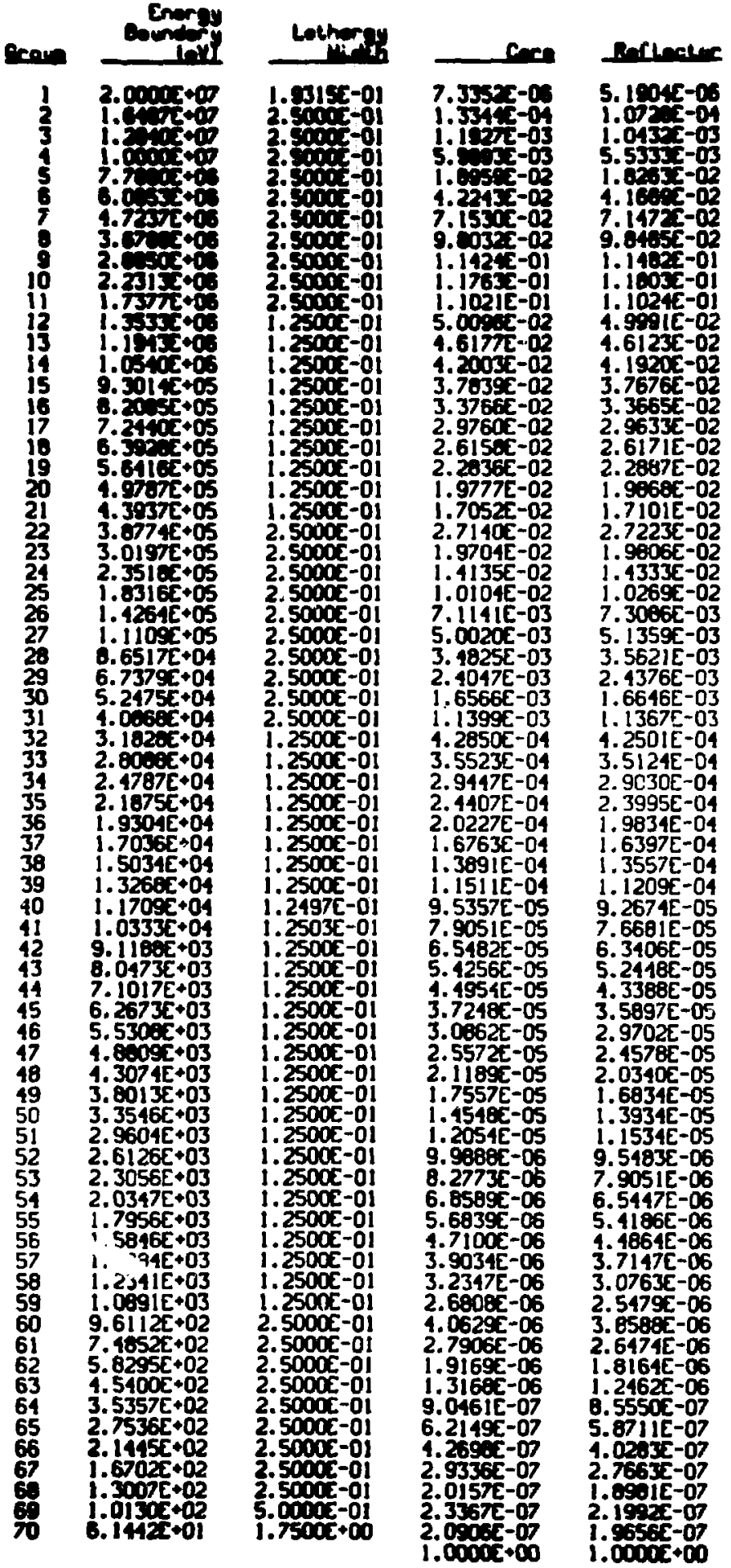


Trier n-XXIV

Puntrop-25 25-chour temwsont treonr srecten

1
3
3
5
8
8
10
11
12
13
14
15
17
17
18
20
21
22
23
24
25

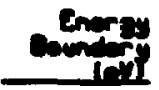

$2.111 \times 40$

$3.5+3+30$

$1.3+1+3+0$

$4.0+4+4$

3.01 rato

$1.0113+00$

1.110 200

$6.73+2 \cdot 01$

1. 100

2. $4 \pi \mathrm{TC}+\mathrm{OM}$

1. $9 x+2+0$

0.116.

$5.51+05$

$3.33+2+03$

2. conte+os

1.2411200

7.4.5.50

$4.3+10+0$

$2.753 \times+02$

1. $70.2+02$

$1.01305+02$

8.14t.2001

$1.057 \pi+01$

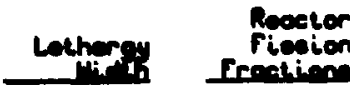

1. $19316+00$

5. $0000-01$

5.0001

5. $0000-01$

5. $0000-01$

5. $000-0$

5.0000-01

5. $0000-0$

5. $0000-0$

5. $\operatorname{cocot}-0$

5. $000 \mathrm{t}-01$

5. $0000 \mathrm{c}-01$

5. $0000-01$

5. $0000-01$

5. $0000 \mathrm{c}-01$

5. $0000 \mathrm{t}-0$

5. $0000 \mathrm{c}-01$

5. $0000 \mathrm{x}-01$

5. $0000 \mathrm{t}-0$

5. $0000 \mathrm{c}-01$

5. $0000 \mathrm{c}-01$

5. $0000 \mathrm{c}-01$

5. $0000 \mathrm{x}-01$
Froetien

2.5035:-02

$1.1351 E-01$

2. $125 x=01$

2. $27535-01$

1. Toole -01

1. $1240-01$

6. $4025=02$

$3.3915-02$

$1.73000-02$

$0.5300-03$

4. $0716-03$

$1.920 \%=03$

$9.04465-04$

4.255.5-04

$2.0023:-04$

$9.4244 E-05$

1.4379z-05

2. $0907 E-05$

$9.8541 E-06$

4.6163z-06

2. $1914 \varepsilon-06$

$1.0336 \mathrm{E}-06$

1. $87765-07$

2. $3022 E-07$

$2.0592 \mathrm{x}-07$
$1.0000 \mathrm{C}+00$

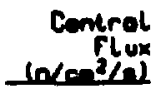

$1.5249 z-02$

$6.493 \pi-02$

$1.31558-01$

i.sic-0

$1.8472-0$

1.549\%-0

1. $2736 x-01$

6. $3595 \mathrm{x}-02$

$4.7202 x-02$

$2.5610 \mathrm{c}-02$

$1.20755-02$

$5.1516 \varepsilon-03$

$1.7046 \varepsilon-03$

$5.7200 \mathrm{c}-04$

1. 8485E-04

6. 3567E-05

2.3161E-05

8. $2043 \mathrm{t}-06$

2. $9605 \varepsilon-06$

$1.0564 E-06$

4.7526 E-07

$1.7639 \mathrm{c}-07$

$8.4977 \mathrm{E}-00$

3. $8288 E-08$

$1.75195-08$

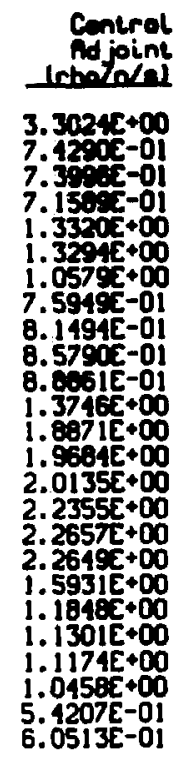

cosom

2.5001E-05

1.050x-04

2.0127e-04

2. olule-ou

7.3510 -04

$1.0375-03$

2. $39335-03$

$2.05332-03$

$1.487 \pi-03$

$1.20075-03$

$9.5546 \bar{c}-04$

4.59-9t-o

$1.51032-04$

$4.2272=05$

$1.27732-05$

4. $03935-06$

$1.1955 E-06$

4. $82735-07$

$1.46155-07$

$7.3206 \mathrm{E}-00$

5. $35335-00$

1.3091E-09

$5.0543 \varepsilon-10$

$1.7694 E-10$

$2.59698-10$ indiotil

$7.705=-00$

1. $25,-02$

i. 0 tite-

$1.1504=6$

1. 1. -1

9. $2004-03$

1. Dile-os

3. $4035 x-03$

2. $25,2-03$

2. $1120-03$

2. 44. $=03$

2.7000 - 05

2.5715E-03

2.7335 $\bar{c}-03$

2. 0372 2-03

3.1911E-03

$4.5419:-03$

3. $0015 \mathrm{E}-0.0$

2. $6201 \mathrm{E}-03$

2.7357t-03

2. $6099 \mathrm{c}-03$

$2.12005-03$

$1.7389-0$

2.6918z-03

TFELE ค-XXY

PLATTOP-25 25-GROUP ZONE FISSION FAnCTIONS

\begin{tabular}{|c|c|c|c|c|}
\hline 29 & $\begin{array}{r}\text { Enurgy } \\
\text { Boundory } \\
\text { (nv) }\end{array}$ & $\begin{array}{c}\text { Lothor } \\
\text { Hiv }\end{array}$ & Core & Bn \\
\hline $\begin{array}{l}1 \\
2 \\
3 \\
4 \\
5 \\
8 \\
7 \\
8 \\
9 \\
10 \\
11 \\
12 \\
13 \\
14 \\
15 \\
16 \\
17 \\
18 \\
19 \\
20 \\
21 \\
22 \\
23 \\
24\end{array}$ & 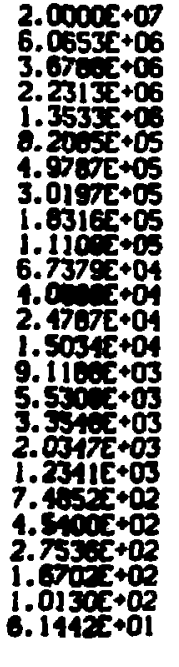 & 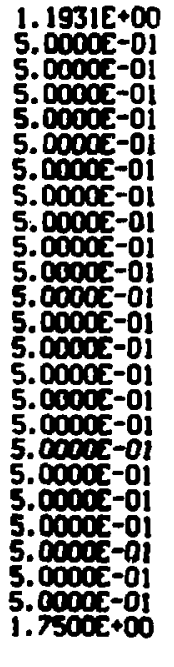 & 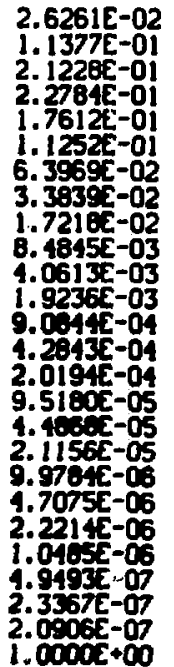 & 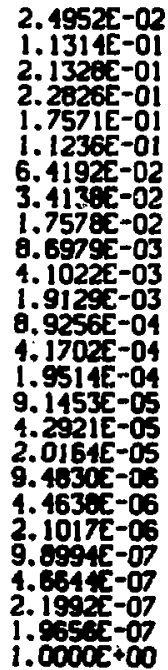 \\
\hline
\end{tabular}




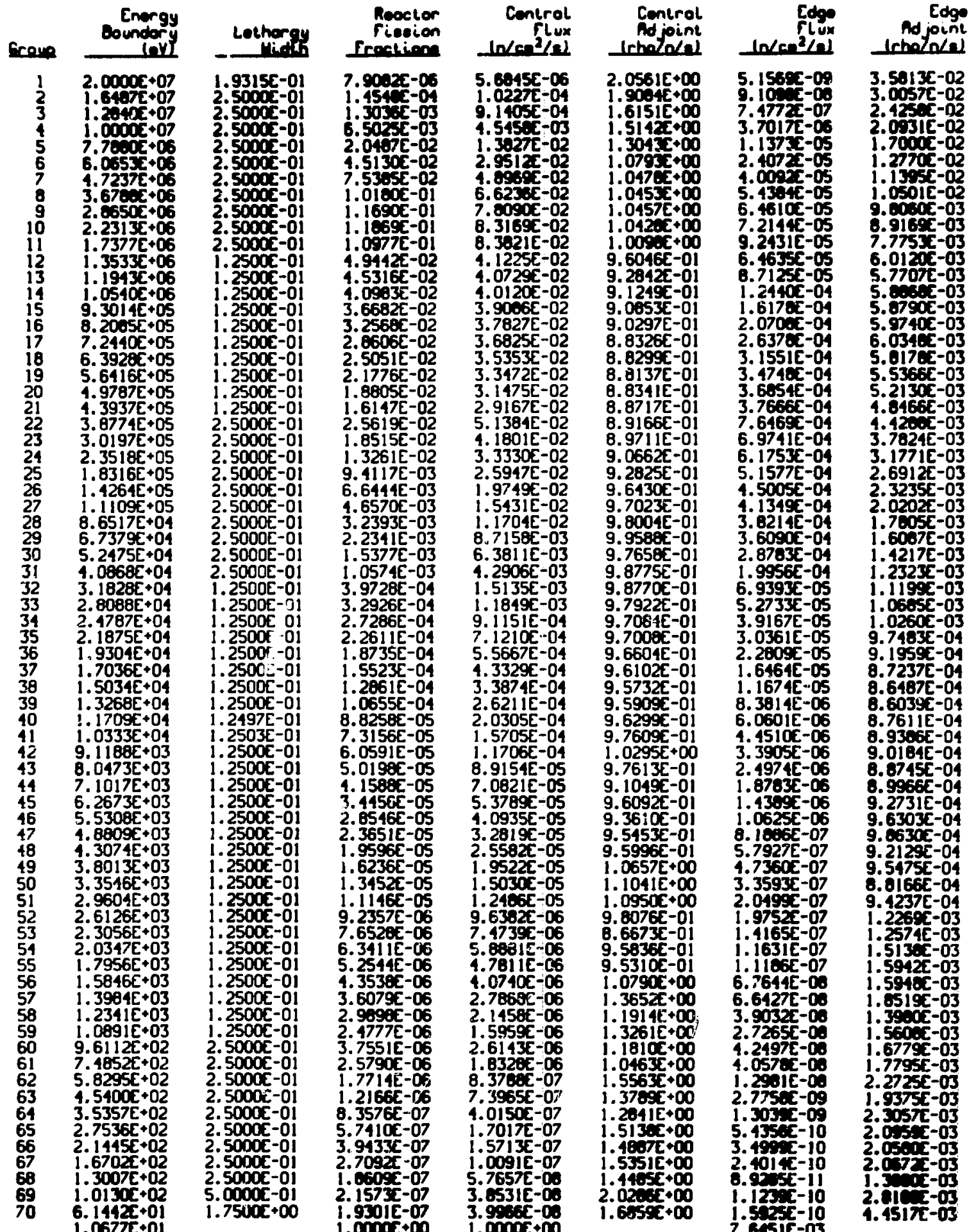


Thus A-XXYII

FLATTOP-Pu 70-enoe zore FIssion renctions

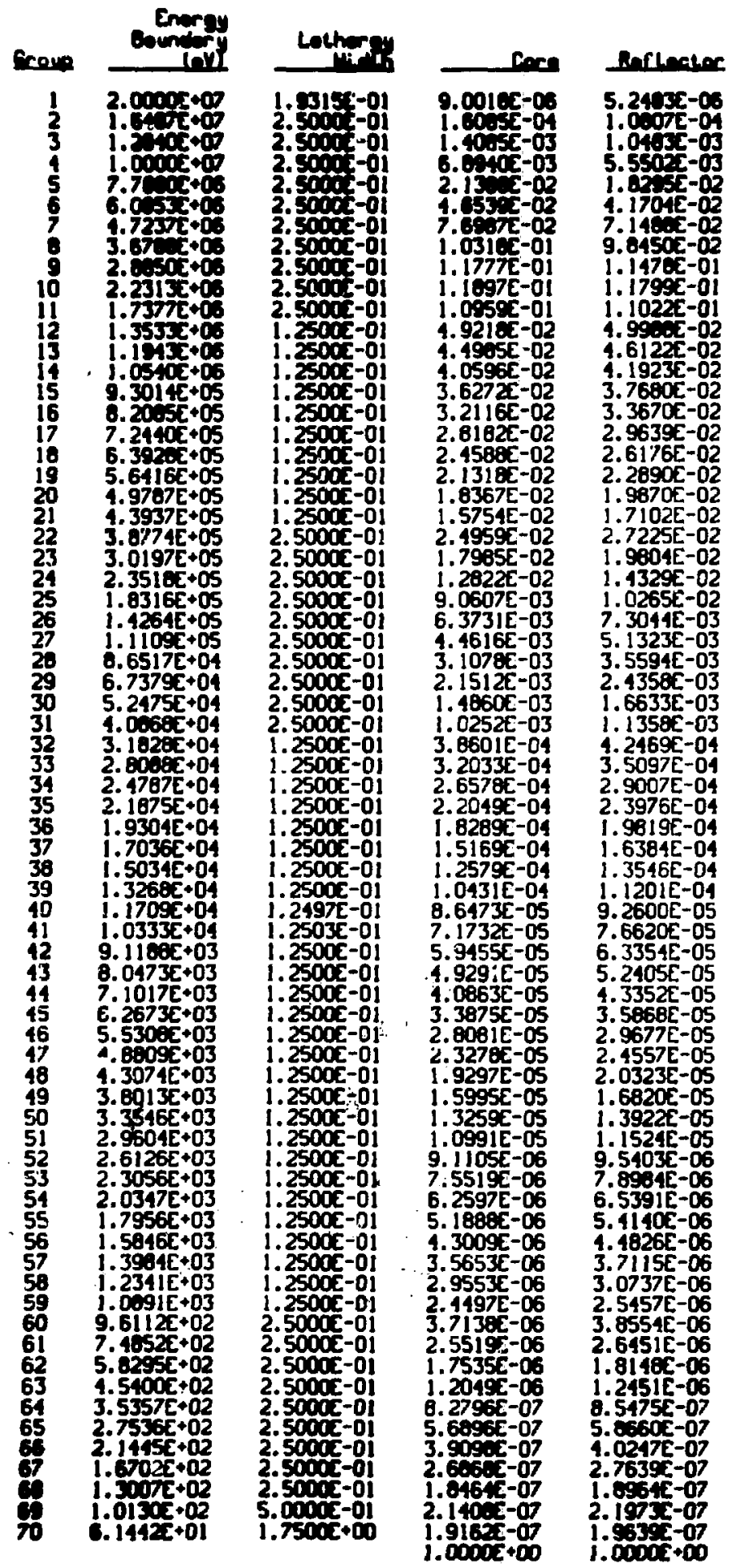


Trace A-XXVIII

FLATTOP-PU 25-GHOUP THAWSPORT THEONY SPECTMA

\begin{tabular}{|c|c|c|c|c|c|c|}
\hline טa & $\begin{array}{r}\text { Energy } \\
\text { Doundory } \\
\text { (nv) }\end{array}$ & $\begin{array}{r}\text { Lelhorgy } \\
\text { Mindth }\end{array}$ & $\begin{array}{l}\text { Re } \\
\text { Fi } \\
\text { Iroc }\end{array}$ & $\begin{array}{r}\text { Central } \\
\text { Flux } \\
\text { Joran } 2 / e l\end{array}$ & $\begin{array}{l}\text { Con } \\
\text { And } \\
\text { into }\end{array}$ & \\
\hline $\begin{array}{r}1 \\
2 \\
3 \\
4 \\
5 \\
6 \\
7 \\
8 \\
9 \\
10 \\
11 \\
12 \\
13 \\
14 \\
15 \\
15 \\
17 \\
18 \\
19 \\
20 \\
21 \\
22 \\
23 \\
24\end{array}$ & 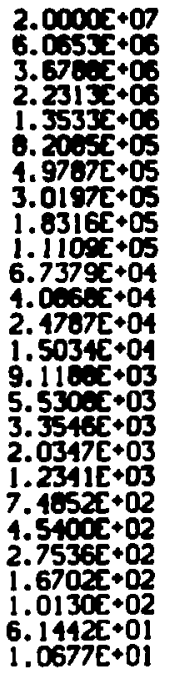 & 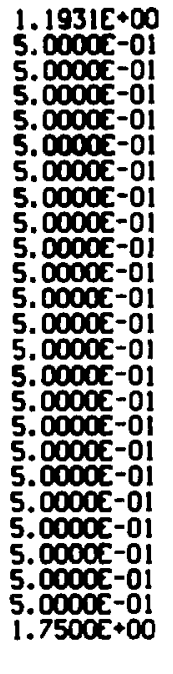 & $\begin{array}{l}2.8447 E-02 \\
1.2051 E-01 \\
2.1870 E-01 \\
2.2046 E-01 \\
1.7242 E-01 \\
1.0000 E-01 \\
6.0571 E-02 \\
3.1777 E-02 \\
1.6056 E-02 \\
7.8963 E-03 \\
3.7718 E-03 \\
1.7839 E-03 \\
6.4154 E-04 \\
3.9658 E-04 \\
1.6603 E-04 \\
8.8028 E-05 \\
4.1487 E-05 \\
1.9557 E-05 \\
9.2226 E-06 \\
1.3504 E-06 \\
2.0524 E-06 \\
9.6042 E-07 \\
4.5702 E-07 \\
2.1573 E-07 \\
1.9301 E-07 \\
1.0000 E+00\end{array}$ & 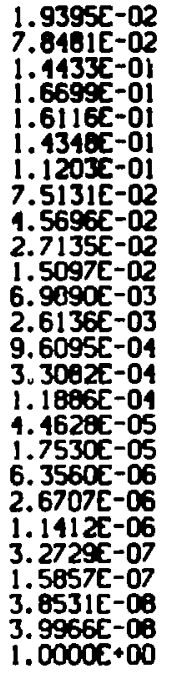 & 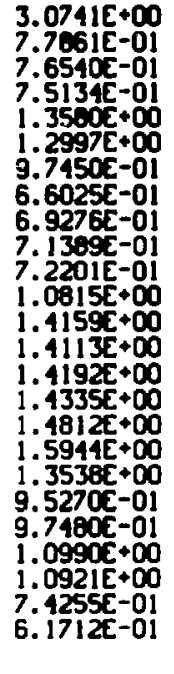 & $\begin{array}{l}9 \\
7 \\
6 \\
3 \\
1 \\
3\end{array}$ \\
\hline
\end{tabular}

anjeine

4. $\cos 2-0$

8.045-03

$7.435=-03$

6.1101E-03

a.c1

8. $5 x+2-0$

$5.303,-03$

2.547. $2=03$

1.00s-03

1.3.13:-03

$1.10932-03$

$1.2521 E-03$

$1.3005-03$

i. $200-03$

$1.323 \pi-03$

1. $4 \cos 2-03$

$1.5770=03$

$2.30035-03$

$1.6975 \mathrm{~s}-03$

$1.403 x-03$

$1.553 x-03$

$1.5205:-03$

1.25752 .03

$1.0311 \mathrm{E}-03$

$1.6295 \mathrm{t}-03$

IfaLE A-XXIX

FLATTOP-PU 25-GROUP ZONE FISSION FRACTIONS

\begin{tabular}{|c|c|c|c|c|}
\hline פ & $\begin{array}{r}\text { Enorgy } \\
\text { Boundory } \\
\text { levl }\end{array}$ & $\begin{array}{l}\text { Lothorgy } \\
\text { Hideh }\end{array}$ & Core & Reflector \\
\hline $\begin{array}{r}0 \\
7 \\
8 \\
9 \\
10 \\
11 \\
12 \\
13\end{array}$ & 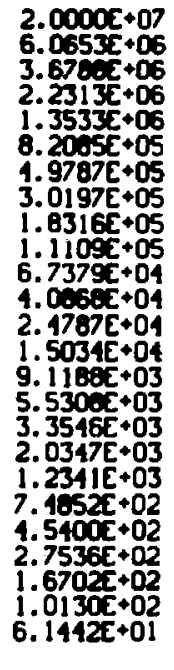 & 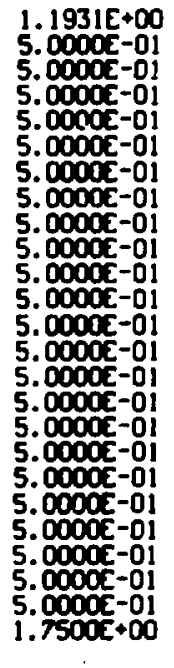 & 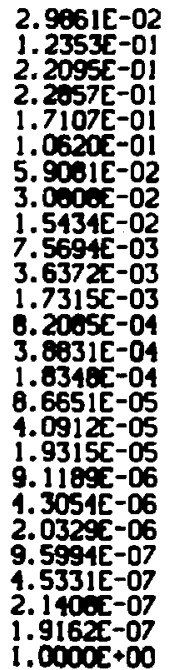 & 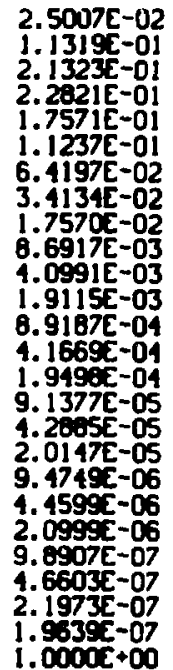 \\
\hline
\end{tabular}




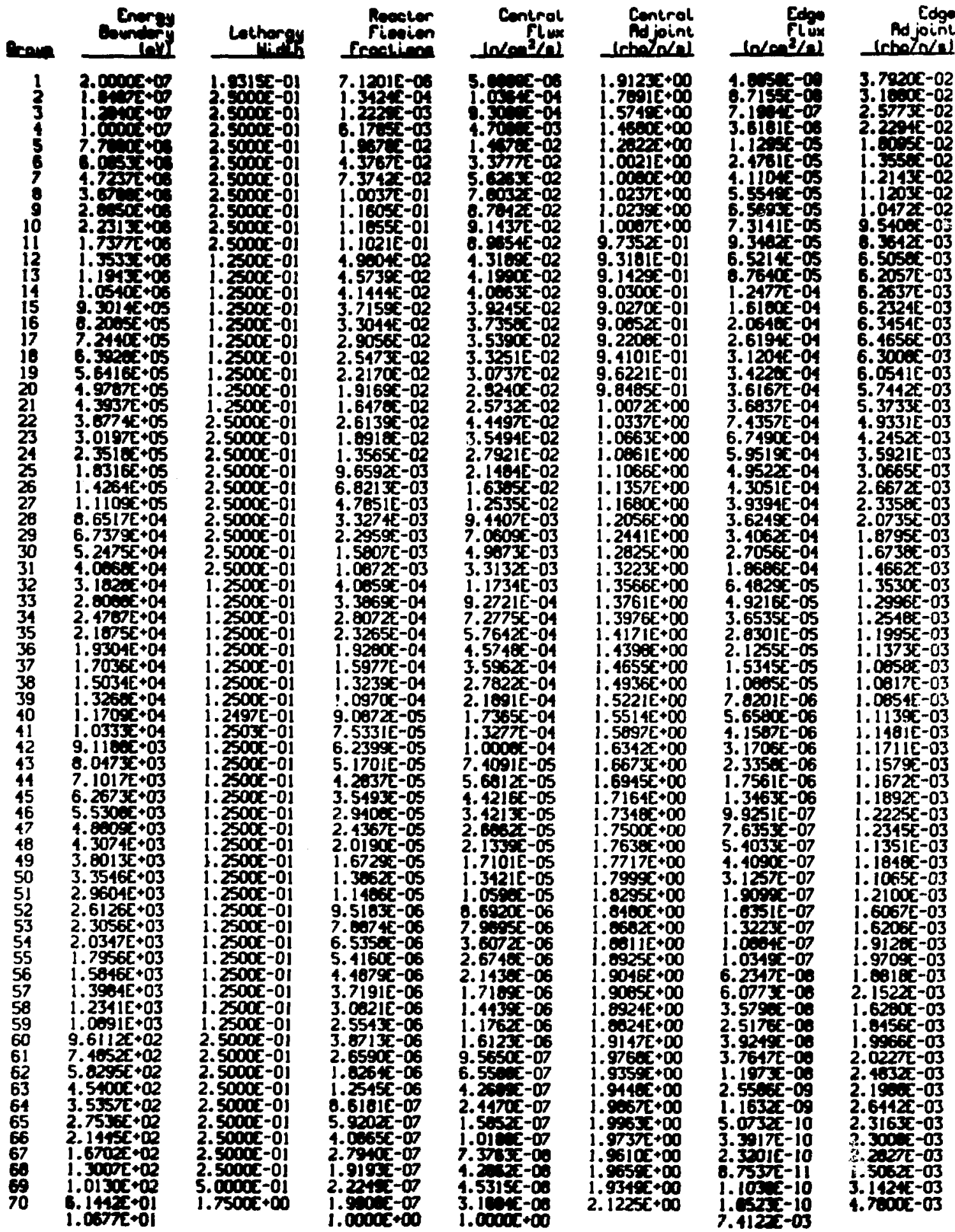


TFELE $\mathrm{A}-\mathrm{XXX1}$

FLATTCP-23 70-GAOU 20NE FISSION FANCTIONS

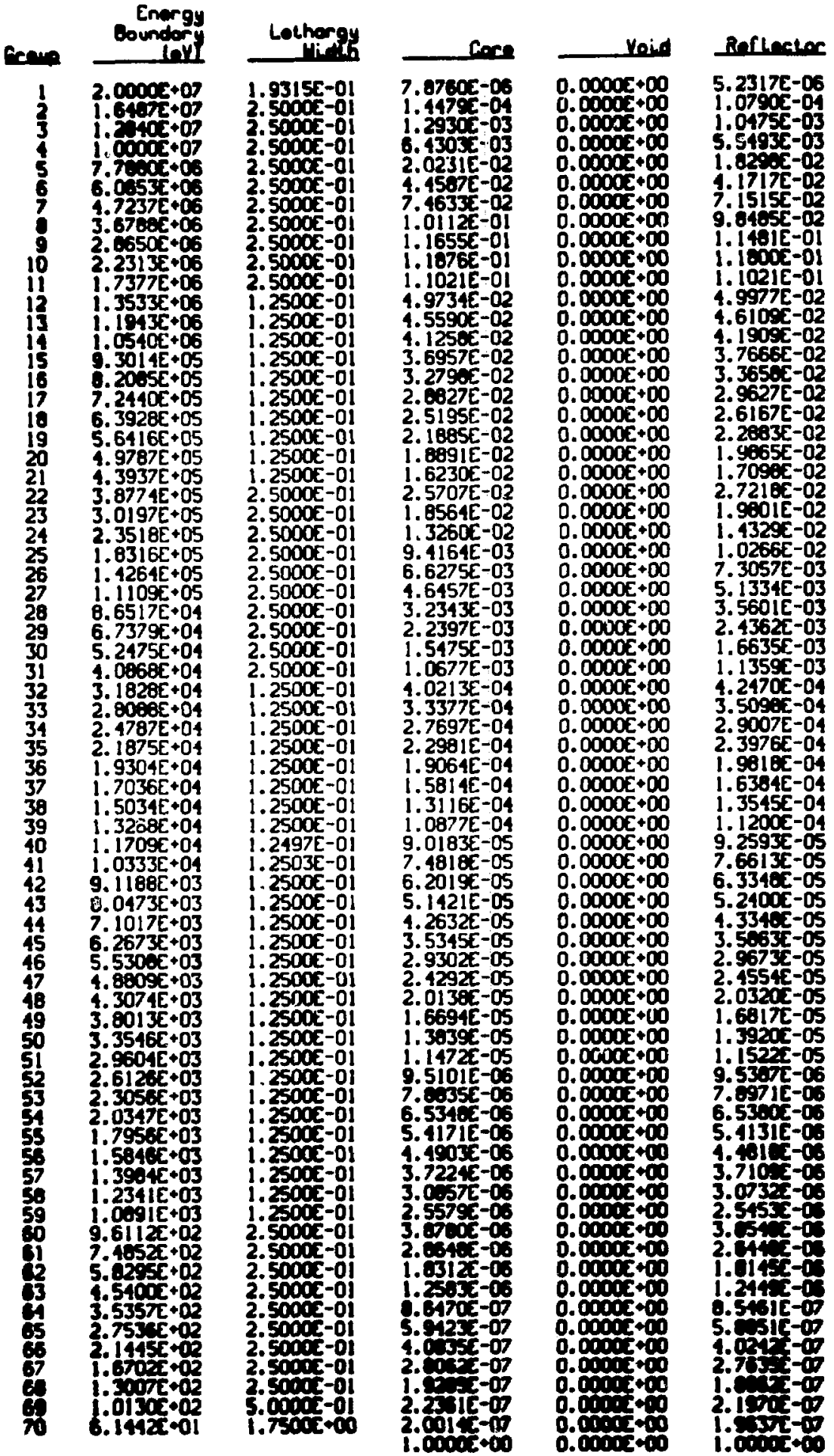


TFELE $A-X \times X \mid V$

THOR 70-GROUP TRENSPORT THEORY SPECTRA

\begin{tabular}{|c|c|c|c|c|c|c|c|}
\hline -oun & $\begin{array}{r}\text { Energy } \\
\text { Boundory } \\
\text { (nY) }\end{array}$ & $\begin{array}{r}\text { Lethorgy } \\
\text { Hidlh }\end{array}$ & $\begin{array}{r}\text { Reoctor } \\
\text { Fiteoion } \\
\text { Eroctionn }\end{array}$ & $\begin{array}{r}\text { Contral } \\
\text { Flux } \\
\ln \left\langle a^{2} /{ }^{2} l\right.\end{array}$ & $\begin{array}{r}\text { Centrol } \\
\text { Ad joint } \\
\text { Irhotolel } \\
\end{array}$ & $\begin{array}{r}\text { Edge } \\
f l u x \\
\ln \left\langle a s^{2} / n\right) \\
\end{array}$ & $\begin{array}{r}\text { Edge } \\
\text { Ad jount } \\
\text { (rho/n/s) } \\
\end{array}$ \\
\hline 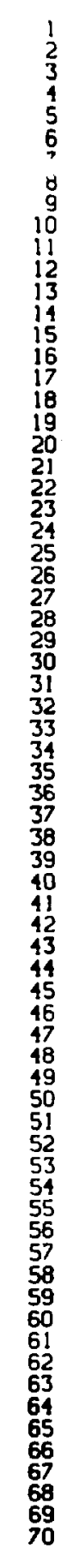 & $\begin{array}{l}2.0000 E+07 \\
1.6487 E+07 \\
1.2840 E+07 \\
1.0000 E+07 \\
7.7860 E+06 \\
6.0653 E+06 \\
4.7237 E+06 \\
3.6788 E+06 \\
2.8650 E+06 \\
2.2313 E+06 \\
1.7377 E+06 \\
1.3533 E+06 \\
1.1943 E+06 \\
1.0540 E+05 \\
9.3014 E+05 \\
8.2085 E+05 \\
7.2440 E+05 \\
6.3928 E+05 \\
5.6416 E+05 \\
4.9787 E+05 \\
4.3937 E+05 \\
3.6774 E+05 \\
3.0197 E+05 \\
2.3518 E+05 \\
1.8316 E+05 \\
1.4264 E+05 \\
1.1109 E+05 \\
8.6517 E+04 \\
6.7379 E+04 \\
5.2475 E+04 \\
4.0868 E+04 \\
3.1828 E+04 \\
2.8088 E+04 \\
2.4787 E+04 \\
2.1875 E+04 \\
1.9304 E+01 \\
1.7036 E+04 \\
1.5034 E+04 \\
1.3268 E+04 \\
1.1709 E+04 \\
1.0333 E+04 \\
9.1188 E+03 \\
8.0473 E+03 \\
7.1017 E+03 \\
6.2673 E+03 \\
5.5308 E+03 \\
4.6809 E+03 \\
4.3074 E+03 \\
3.8013 E+03 \\
3.3546 E+03 \\
2.9604 E+03 \\
2.6126 E+03 \\
2.3056 E+03 \\
2.0347 E+03 \\
1.7956 E+03 \\
1.5846 E+03 \\
1.3984 E+03 \\
1.2341 E+03 \\
1.0891 E+03 \\
9.6112 E+02 \\
7.4852 E+02 \\
5.8295 E+02 \\
4.5400 E+02 \\
3.5357 E+02 \\
2.7536 E+02 \\
2.1445 E+02 \\
1.6702 E+02 \\
1.3007 E+02 \\
1.0130 E+02 \\
1\end{array}$ & $\begin{array}{l}1.9315 E-01 \\
2.5000 E-01 \\
2.5000 E-01 \\
2.5000 E-01 \\
2.5000 E-01 \\
2.5000 E-01 \\
2.5000 E-01 \\
2.5000 E-01 \\
2.5000 E-01 \\
2.5000 E-01 \\
2.5000 E-01 \\
1.2500 E-01 \\
1.2500 E-01 \\
1.2500 E-01 \\
1.2500 E-01 \\
1.2500 E-01 \\
1.2500 E-01 \\
1.2500 E-01 \\
1.2500 E-01 \\
1.2500 E-01 \\
1.2500 E-01 \\
2.5000 E-01 \\
2.5000 E-01 \\
2.5000 E-01 \\
2.5000 E-01 \\
2.5000 E-01 \\
2.5000 E-01 \\
2.5000 E-01 \\
2.5000 E-01 \\
2.5000 E-01 \\
2.5000 E-01 \\
1.2500 E-01 \\
1.2500 E-01 \\
1.2500 E-01 \\
1.2500 E-01 \\
1.2500 E-01 \\
1.2500 E-01 \\
1.2500 E-01 \\
1.2500 E-01 \\
1.2497 E-01 \\
1.2503 E-01 \\
1.2500 E-01 \\
1.2500 E-01 \\
1.2500 E-01 \\
1.2500 E-01 \\
1.2500 E-01 \\
1.2500 E-01 \\
1.2500 E-01 \\
1.2500 E-01 \\
1.2500 E-01 \\
1.2500 E-01 \\
1.2500 E-01 \\
1.2500 E-01 \\
1.2500 E-01 \\
1.2500 E-01 \\
1.2500 E-01 \\
1.2500 E-01 \\
1.2500 E-01 \\
1.2500 E-01 \\
2.5000 E-01 \\
2.5000 E-01 \\
2.5000 E-01 \\
2.5000 E-01 \\
2.5000 E-01 \\
2.5000 E-01 \\
2.5000 E-01 \\
2.5000 E-01 \\
2.5000 E-01 \\
5.0000 E-01 \\
1.7500 E+00\end{array}$ & 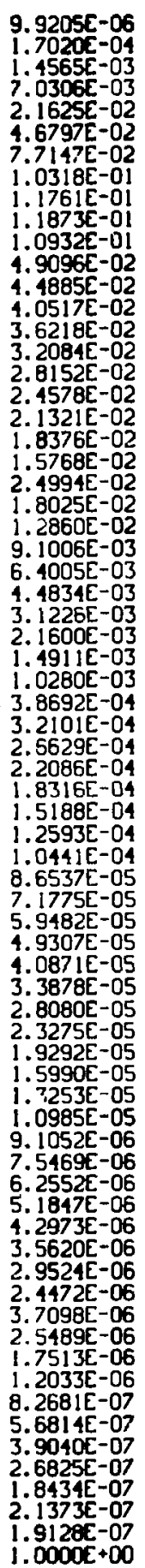 & $\begin{array}{l}\text { 6.1012E-06 } \\
1.0957 E-04 \\
9.7734 E-04 \\
4.8525 E-03 \\
1.4673 E-02 \\
3.1169 E-02 \\
5.1630 E-02 \\
6.9710 E-02 \\
8.2136 E-02 \\
8.7310 E-02 \\
8.7529 E-02 \\
1.2704 E-02 \\
4.1893 E-02 \\
4.0690 E-02 \\
3.9163 E-02 \\
3.7712 E-02 \\
3.6519 E-02 \\
3.4692 E-02 \\
3.2643 E-02 \\
3.0419 E-02 \\
2.7966 E-02 \\
4.8582 E-02 \\
3.8992 E-02 \\
3.0997 E-02 \\
2.4952 E-02 \\
1.9088 E-02 \\
1.4183 E-02 \\
9.9034 E-03 \\
6.5435 E-03 \\
4.3848 E-03 \\
2.8637 E-03 \\
1.0190 E-03 \\
8.1650 E-04 \\
6.4669 E-04 \\
5.1683 E-04 \\
4.1605 E-04 \\
3.3569 E-04 \\
2.7232 E-04 \\
2.1602 E-04 \\
1.7243 E-04 \\
1.3504 E-04 \\
1.0081 E-04 \\
7.6595 E-05 \\
6.2648 E-05 \\
4.7040 E-05 \\
3.5959 E-05 \\
3.0538 E-05 \\
2.3329 E-05 \\
1.7594 E-05 \\
1.3663 E-05 \\
1.1673 E-05 \\
9.0158 E-06 \\
6.9802 E-06 \\
5.5486 E-06 \\
4.4423 E-06 \\
3.8805 E-06 \\
2.6151 E-06 \\
2.0440 E-06 \\
1.5324 E-06 \\
2.5323 E-06 \\
1.7306 E-06 \\
8.1633 E-07 \\
7.4498 E-07 \\
4.0385 E-07 \\
1.7068 E-07 \\
1.5919 E-07 \\
1.0106 E-07 \\
5.7990 E-08 \\
3.8688 E-08 \\
4.0196 E-08 \\
1.0000 E+00\end{array}$ & $\begin{array}{l}1.9514 E+00 \\
1.8169 E+00 \\
1.5317 E+00 \\
1.1452 E+00 \\
1.2501 E+00 \\
1.0516 E+00 \\
1.0295 E+00 \\
1.0335 E+00 \\
1.0377 E+00 \\
1.0347 E+00 \\
1.0089 E+00 \\
9.7824 E-01 \\
9.4877 E-01 \\
9.3546 E-01 \\
9.3018 E-01 \\
9.2243 E-01 \\
9.0209 E-01 \\
9.0248 E-01 \\
9.0069 E-01 \\
9.0248 E-01 \\
9.0613 E-01 \\
9.0927 E-01 \\
9.1266 E-01 \\
9.2051 E-01 \\
9.4297 E-01 \\
9.8267 E-01 \\
9.9142 E-01 \\
1.0030 E+00 \\
1.0213 E+00 \\
1.0035 E+00 \\
1.0156 E+00 \\
1.0148 E+00 \\
1.0058 E+00 \\
9.9626 E-01 \\
9.9544 E-01 \\
9.9178 E-01 \\
9.8690 E-01 \\
9.8251 E-01 \\
9.8358 E-01 \\
9.8686 E-01 \\
9.9909 E-01 \\
1.0521 E+00 \\
9.9669 E-01 \\
9.2836 E-01 \\
9.7852 E-01 \\
9.5071 E-01 \\
9.6689 E-01 \\
9.6855 E-01 \\
1.0884 E+00 \\
1.1230 E+00 \\
1.1109 E+00 \\
9.9953 E-01 \\
8.7883 E-01 \\
9.7663 E-01 \\
9.6900 E-01 \\
1.0920 E+00 \\
1.3788 E+00 \\
1.2077 E+00 \\
1.3397 E+00 \\
1.2024 E+00 \\
1.0480 E+00 \\
1.5615 E+00 \\
1.3820 E+00 \\
1.2786 E+00 \\
1.5072 E+00 \\
1.4904 E+00 \\
1.5339 E+00 \\
1.4422 E+00 \\
2.0188 E+00 \\
1.6759 E+00\end{array}$ & 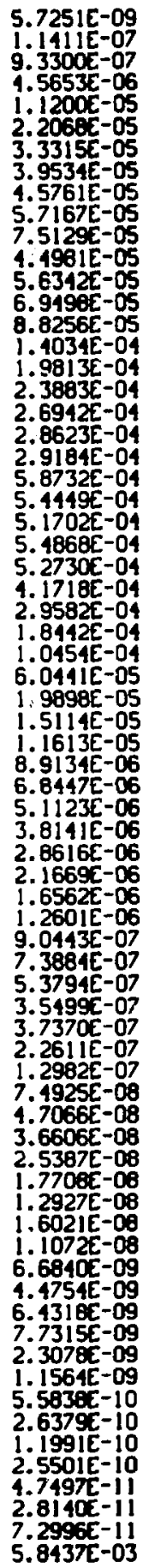 & 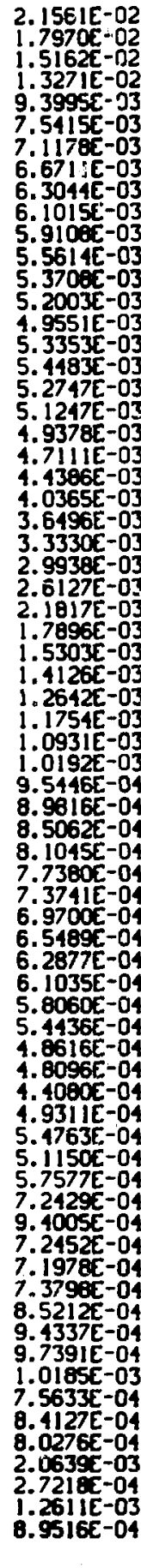 \\
\hline
\end{tabular}


Thue $ค-x \times x y$

\section{Thon 70-cmour zond fission fanctions}

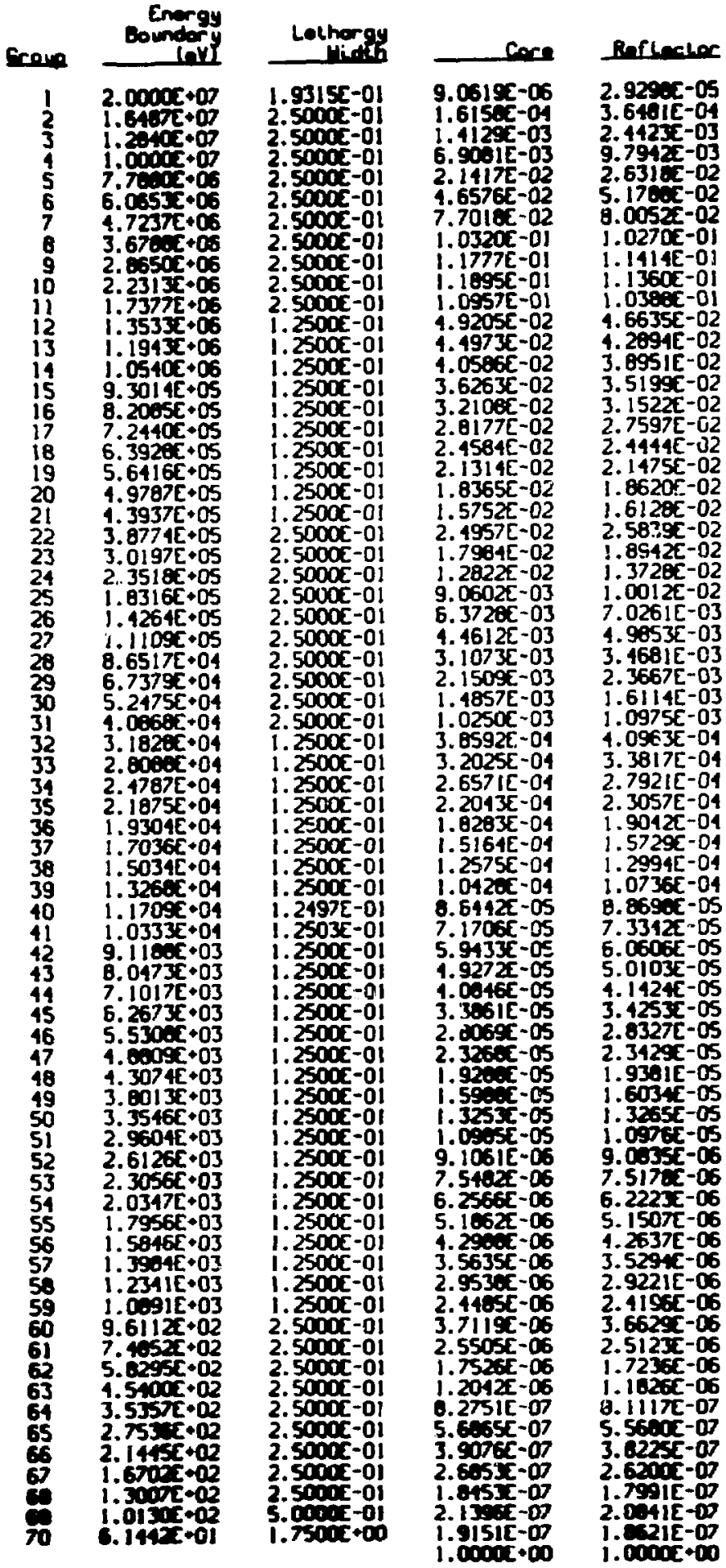


TRELE A-XXXYI

THOR 25-GROP TRANSPORT THEORY SHECTRA

\begin{tabular}{|c|c|c|c|c|c|c|}
\hline ang & $\begin{array}{r}\text { Energy } \\
\text { Boundory } \\
\text { IeVI] }\end{array}$ & $\begin{array}{r}\text { Lolhorgy } \\
\text { Hidtuh }\end{array}$ & $\begin{array}{r}\text { Reoctor } \\
\text { Fiseion } \\
\text { froctione }\end{array}$ & $\begin{array}{r}\text { Control } \\
\text { Flux } \\
\ln / \mathrm{an}^{2} / \mathrm{al} \\
\end{array}$ & $\begin{array}{r}\text { Centrol } \\
\text { Ad joint } \\
\text { trhotoulel }\end{array}$ & \\
\hline $\begin{array}{l}1 \\
2 \\
3 \\
4 \\
5 \\
6 \\
7 \\
6 \\
9 \\
10 \\
11 \\
12 \\
13 \\
14 \\
15 \\
16 \\
17 \\
18 \\
19 \\
20 \\
21 \\
22 \\
23 \\
24 \\
25\end{array}$ & 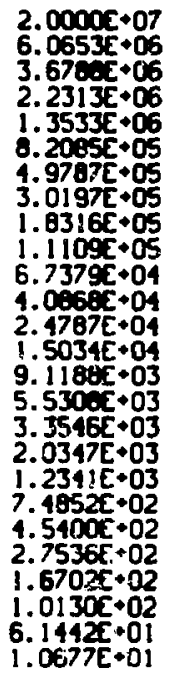 & 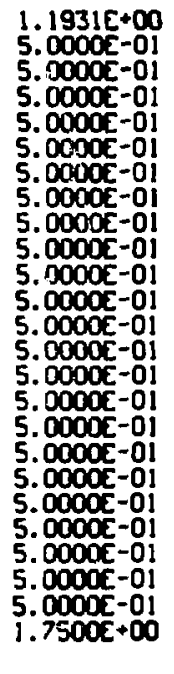 & $\begin{array}{l}3.0292 E-02 \\
1.2394 E-01 \\
2.2079 E-01 \\
2.2005 E-01 \\
1.7072 E-01 \\
1.0613 E-01 \\
5.9136 E-02 \\
3.0655 E-02 \\
1.5501 E-02 \\
7.6060 E-03 \\
3.6511 E-03 \\
1.7360 E-03 \\
8.2218 E-04 \\
3.8065 E-04 \\
1.8354 E-04 \\
8.6637 E-05 \\
1.0991 E-05 \\
1.9299 E-05 \\
9.1095 E-06 \\
4.3002 E-06 \\
2.0301 E-06 \\
9.5554 E-07 \\
4.5259 E-07 \\
2.1373 E-07 \\
1.9120 E-07 \\
1.0000 E+00\end{array}$ & 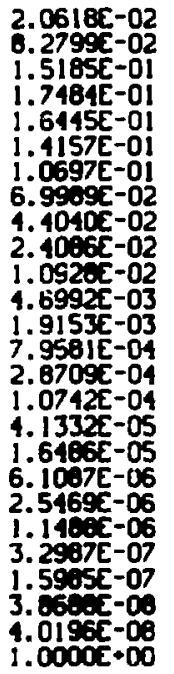 & 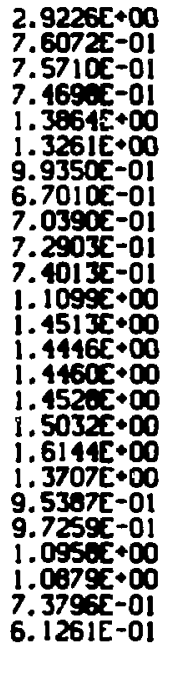 & 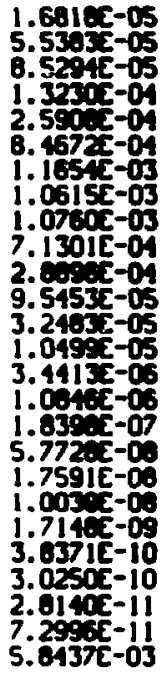 \\
\hline
\end{tabular}

$\begin{array}{r}\text { Edge } \\ \text { ichoingint } \\ \hline\end{array}$

2. $027:-02$

$5.350+2-03$

$4.7431 E-03$

4. $3910 \mathrm{c}-03$

$7.70 x-03$

$7.743 \mathrm{x}-03$

5.14.t-03

2. $00 .+5-03$

2. $3127-03$

$1.7575-03$

$1.2136-03$

$1.4002 x-03$

$1.4493:-03$

i. $15: x-03$

9.4712E-04

$7.6475-04$

$7.205+2-04$

1.0.37t-03

8.1436E-04

7.00.se-0

6. $40795-04$

6. $\cos 5-04$

$8.5393 x-04$

4. $600 \mathrm{c}=04$

3.2722 -04

Tfare A-xxxv!I

Thon 25-Gene 20ic fission fenctions

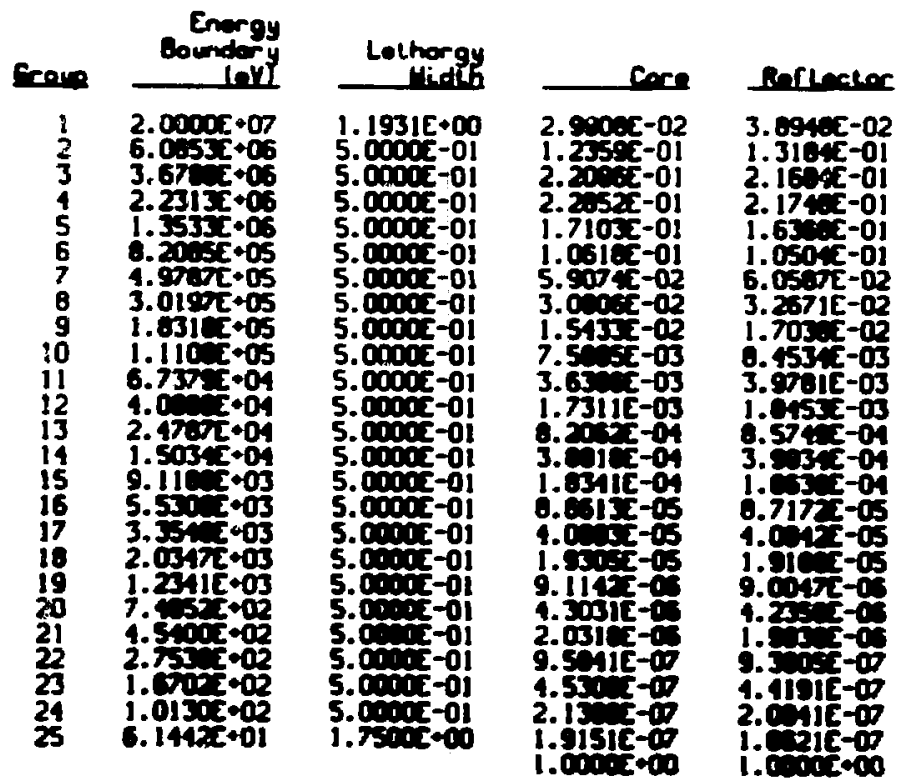


IFBLE A-XXXVIII

JEZEBEL CENTRAL AVERAGE CROSS SECTIONS

\begin{tabular}{|c|c|c|c|c|}
\hline heris & Finsion & Cooture & Nu & Irompore \\
\hline $\begin{array}{l}P U-239 \\
P U-240 \\
P U-241 \\
6 A \\
U-238 \\
U-235 \\
U-233 \\
N P-237 \\
V \\
M N-55 \\
C U \\
N B-93 \\
A U-197 \\
H-1 \\
B E-9 \\
B-10 \\
C \\
N-14 \\
O-16 \\
A-27 \\
T I \\
F E \\
C D-59 \\
N ! \\
2 R \\
M O \\
A G-107 \\
A G-109 \\
T A-181 \\
W-182 \\
W-183 \\
W-184 \\
W-186 \\
T H-232 \\
A N-241\end{array}$ & $\begin{array}{l}1.75579 E+00 \\
1.19034 E+00 \\
1.62250 E+00 \\
0.00000 E+00 \\
2.44328 E-01 \\
1.24699 E+00 \\
1.94137 E+00 \\
1.18657 E+00 \\
0.00000 E+00 \\
0.00000 E+00 \\
0.00000 E+00 \\
0.00000 E+00 \\
0.00000 E+00 \\
0.00000 E+00 \\
0.00000 E+00 \\
0.00000 E+00 \\
0.00000 E+00 \\
0.00000 E+00 \\
0.00000 E+00 \\
0.00000 E+00 \\
0.00000 E+00 \\
0.00000 E+00 \\
0.00000 E+00 \\
0.00000 E+00 \\
0.00000 E+00 \\
0.00000 E+00 \\
0.00000 E+00 \\
0.00000 E+00 \\
0.00000 E+00 \\
0.00000 E+00 \\
0.00000 E+00 \\
0.00000 E+00 \\
0.00000 E+00 \\
6.04543 E-02 \\
1.23895 E+00\end{array}$ & $\begin{array}{l}5.77883 E-02 \\
1.08830 E-01 \\
1.05567 E-01 \\
3.12996 E-02 \\
6.74352 E-02 \\
1.17241 E-01 \\
6.96737 E-02 \\
2.40580 E-01 \\
2.22275 E-03 \\
3.53936 E-03 \\
1.04490 E-02 \\
3.38166 E-02 \\
9.86331 E-02 \\
1.56915 E-05 \\
-6.66598 E-02 \\
5.54970 E-01 \\
1.69424 E-03 \\
1.03117 E-01 \\
7.51183 E-03 \\
4.85717 E-03 \\
5.62303 E-03 \\
8.65849 E-03 \\
8.27693 E-03 \\
6.84930 E-02 \\
9.01648 E-03 \\
2.97271 E-02 \\
1.25732 E-01 \\
1.37477 E-01 \\
1.22270 E-01 \\
5.35738 E-02 \\
2.61822 E-02 \\
3.91364 E-02 \\
3.11573 E-02 \\
9.12867 E-02 \\
3.59570 E-01\end{array}$ & $\begin{array}{l}3.15322 E+00 \\
3.14921 E+00 \\
3.14429 E+00 \\
0.00000 E+00 \\
2.82424 E+00 \\
2.62634 E+00 \\
2.66345 E+00 \\
3.03397 E+00 \\
0.00000 E+00 \\
0.00000 E+00 \\
0.00000 E+00 \\
0.00000 E+00 \\
0.00000 E+00 \\
0.00000 E+00 \\
0.00000 E+00 \\
0.00000 E+00 \\
0.00000 E+00 \\
0.00000 E+00 \\
0.00000 E+00 \\
0.00000 E+00 \\
0.00000 E+00 \\
0.00000 E+00 \\
0.00000 E+00 \\
0.00000 E+00 \\
0.00000 E+00 \\
0.00000 E+00 \\
0.00000 E+00 \\
0.00000 E+00 \\
0.00000 E+00 \\
0.00000 E+00 \\
0.00000 E+00 \\
0.00000 E+00 \\
0.00000 E+00 \\
2.14657 E+00 \\
3.49327 E+00\end{array}$ & 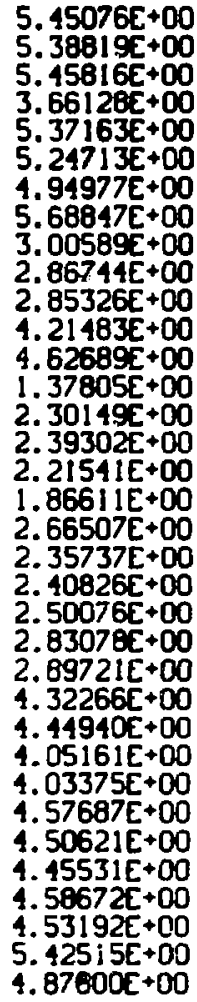 \\
\hline
\end{tabular}

TFaLE $A-X X X] X$

GOOIVA CENTRAL AVERPEE CROSS SECTIONS

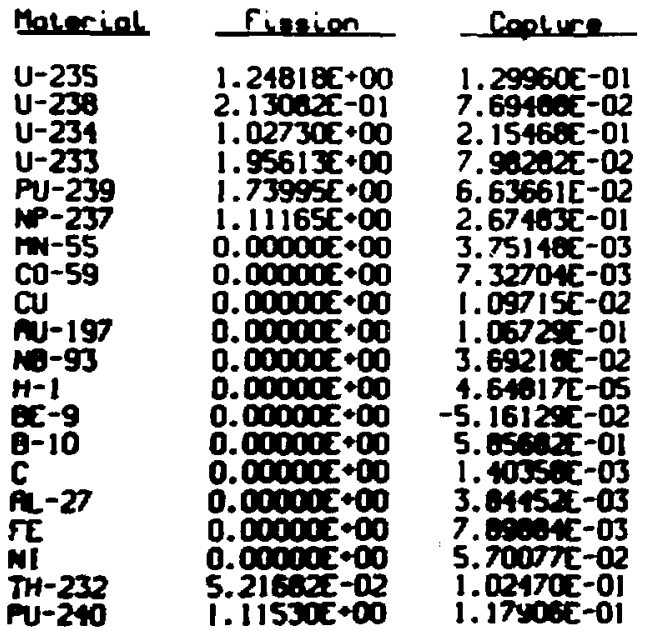

$\mathrm{Nu}$

2. $60206 \tau+00$

2. $80395 E+00$

$2.63279 \mathrm{C}+00$

$2.63936 c \cdot 00$

$3.12300 \mathrm{C} \cdot 00$

3. $010055+00$

$0.00000 \mathrm{C} \cdot 00$

$0.00000 \Sigma+00$

$0.00000 c+00$

$0.00000 \tau+00$

$0.000002 \cdot 00$

$0.000002+\infty 0$

$0.00000 \bar{c}+\infty 0$

$0.000002+\infty 0$

$0.00000 \mathrm{C}+00$

$0.00000 \mathrm{C} \cdot 00$

$0.00000 \mathrm{D}+00$

0.00000 e.00

$2.424355 \cdot 00$

$3.12461 E \cdot 00$
Ironsport

5. $35282 E+\infty 0$

$5.477221+\infty 0$

$6.331905+00$

$5.02313 \mathrm{E}+00$

$5.54763 \mathrm{E} \cdot 00$

$5.764665+\infty$

2. $90205 E+\infty 0$

2. $00090 \mathrm{C}+00$

$2.90733 x+\infty$

$4.69441 \mathrm{E}+00$

4. $36046 \mathrm{~T}+00$

$1.440 .2 x+00$

2. $3951 \pi+00$

2. $49574+00$

$2.2041 \pi+00$

$2.43450 .2 \cdot 00$

2.53210:-00

2.95302 -00

$5.54525 \% \cdot 00$

5. $40241 E+00$ 
TABLE A-XL

JEZEBEL-23 CENTRAL AVERAGE CRDSS SECTIONS

\begin{tabular}{|c|c|c|c|c|}
\hline Moteriol & Fiscion & Copture & $\mathrm{Nu}_{4}$ & Tronempert \\
\hline $\begin{array}{l}U-233 \\
U-234 \\
U-235 \\
U-239 \\
N P-237 \\
P-31 \\
\text { RL-27 } \\
F E \\
C U\end{array}$ & $\begin{array}{l}1.93123 E+00 \\
1.12913 E+00 \\
1.24195 E+00 \\
2.63989 E-01 \\
1.23261 E+00 \\
0.00000 E+00 \\
0.00000 E+00 \\
0.00000 E+00 \\
0.00000 E+00\end{array}$ & $\begin{array}{l}6.37848 E-02 \\
1.93555 E-01 \\
1.09838 E-01 \\
6.34696 \mathrm{E}-02 \\
2.21975 \mathrm{E}-01 \\
3.32225 \mathrm{E}-02 \\
5.09178 \mathrm{E}-03 \\
6.86064 \mathrm{E}-03 \\
2.07291 \mathrm{E}-02\end{array}$ & $\begin{array}{l}2.67631 E+00 \\
2.66508 E+00 \\
2.63819 E+00 \\
2.62423 E+00 \\
3.04319 E+00 \\
0.00000 E+00 \\
0.00000 E+00 \\
0.00000 E+00 \\
0.00000 E+00\end{array}$ & $\begin{array}{l}1.86727 E+\infty 0 \\
6.10461 E+\infty 0 \\
5.14947 E+\infty 0 \\
5.26797 E+\infty 0 \\
5.61432 E+\infty 0 \\
2.06988 E+\infty 0 \\
2.29439 E+\infty 0 \\
2.16260 E+\infty 0 \\
2.79371 E+\infty 0\end{array}$ \\
\hline
\end{tabular}

TABLE A-XLI

BIGTEN CENTRAL AVERAGE CROSS SECTIONS

\begin{tabular}{|c|c|c|c|c|}
\hline ial & $\mathrm{Fis}_{\mathrm{i}}$ & Copture & Nv & Ironaport \\
\hline $\begin{array}{l}-234 \\
-235 \\
-238 \\
0-237 \\
j-239 \\
-233 \\
-6 \\
-10 \\
-27 \\
j-59 \\
j-197 \\
-1 \\
-7 \\
-9 \\
-11 \\
-16 \\
1-232 \\
-238\end{array}$ & $\begin{array}{l}1.85378 E-01 \\
1.36153 E+00 \\
5.13731 E-02 \\
1.8711 \mathrm{i}-01 \\
1.62269 E+00 \\
2.12360 E+00 \\
0.00000 E+00 \\
0.00000 E+00 \\
0.00000 E+00 \\
0.00000 E+00 \\
0.00000 E+00 \\
0.00000 E+00 \\
0.00000 E+00 \\
0.00000 E+00 \\
0.00000 E+00 \\
0.00000 E+00 \\
0.00000 E+00 \\
0.00000 E+00 \\
0.00000 E+00 \\
1.31182 E-02 \\
1.22123 E+00\end{array}$ & $\begin{array}{r}3.12138 \mathrm{E}-01 \\
2.70796 \mathrm{E}-01 \\
1.46774 \mathrm{E}-01 \\
6.71579 \mathrm{E}-01 \\
1.72533 \mathrm{E}-01 \\
1.62719 \mathrm{E}-01 \\
8.14854 \mathrm{E}-01 \\
1.16875 \mathrm{E}+00 \\
2.13392 \mathrm{E}-03 \\
1.23459 \mathrm{E}-02 \\
1.9416 \mathrm{E}-02 \\
2.09226 \mathrm{E}-01 \\
7.48858 \mathrm{E}-05 \\
8.34648 \mathrm{E}-07 \\
-1.16375 \mathrm{E}-02 \\
1.52962 \mathrm{E}-04 \\
8.18782 \mathrm{E}-04 \\
1.36485 \mathrm{E}-03 \\
7.96683 \mathrm{E}-03 \\
1.78212 \mathrm{E}-01 \\
3.66940 \mathrm{E}-01\end{array}$ & $\begin{array}{l}2.53917 E+00 \\
2.49333 E+00 \\
2.78958 E+00 \\
2.91766 E+00 \\
2.97601 E+00 \\
2.53515 E+00 \\
0.00000 E+00 \\
0.00000 E+00 \\
0.00000 E+00 \\
0.00000 E+00 \\
0.00000 E+00 \\
0.00000 E+00 \\
0.00000 E+00 \\
0.00000 E+00 \\
0.00000 E+00 \\
0.00000 E+00 \\
0.00000 E+00 \\
0.00000 E+00 \\
0.00000 E+00 \\
2.11145 E+00 \\
3.03359 E+00\end{array}$ & 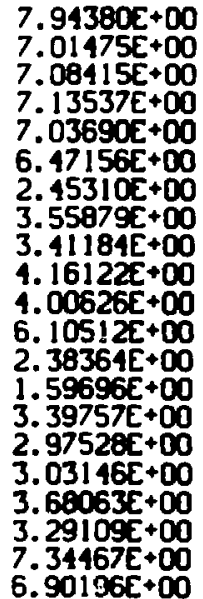 \\
\hline
\end{tabular}

IFBLE A-XLII

JEZEECL-PU CENTRAL AVERACE CROSS SECTIONS

\begin{tabular}{|c|c|c|c|c|}
\hline encial & Fintion & Copture & $\mathrm{Nu}$ & 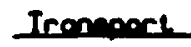 \\
\hline $\begin{array}{l}P U-239 \\
P U-240 \\
P U-241 \\
P U-242 \\
\text { ER } \\
U-230 \\
U-235 \\
N-237 \\
P U-238 \\
C H-244\end{array}$ & 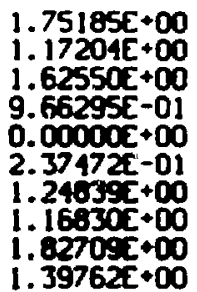 & 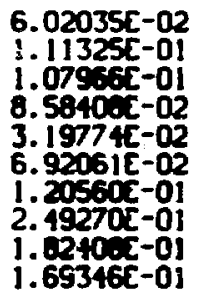 & 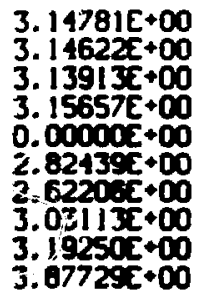 & \\
\hline
\end{tabular}


TABLE A-XLIII

FLATTOP-25 CENTRPL AVERAGE CROSS SECTIONS

\begin{tabular}{|c|c|c|c|c|}
\hline terial & Fireicon & Cop & $\mathrm{Nu}$ & Transport. \\
\hline $\begin{array}{l}U-234 \\
U-235 \\
U-238 \\
U-233 \\
N P-237 \\
P U-239 \\
P U-238 \\
P U-242 \\
P M-241 \\
P-31 \\
P L-27 \\
F E\end{array}$ & $\begin{array}{l}9.64841 E-01 \\
1.25842 E+00 \\
1.94666 E-01 \\
1.97348 E+00 \\
1.03910 E+00 \\
1.72567 E+00 \\
1.71655 E+00 \\
8.52602 E-01 \\
1.04594 E+00 \\
0.00000 E+00 \\
0.00000 E+00 \\
0.00000 E+00 \\
0.00000 E+00\end{array}$ & $\begin{array}{l}2.25317 E-01 \\
1.44558 E-01 \\
8.39212 E-02 \\
8.90122 E-02 \\
3.08488 E-01 \\
7.73638 E-02 \\
2.12988 E-01 \\
1.01682 E-01 \\
4.51398 E-01 \\
2.36714 E-02 \\
3.62882 E-03 \\
7.87779 E-03 \\
1.91806 E-02\end{array}$ & $\begin{array}{l}2.62726 E+00 \\
2.58894 E+00 \\
2.80366 E+00 \\
2.62655 E+00 \\
3.00508 E+00 \\
3.10724 E+00 \\
3.15767 E+00 \\
3.13040 E+00 \\
3.46714 E+00 \\
0.00000 E+00 \\
0.00000 E+00 \\
0.00000 E+00 \\
0.00000 E+00\end{array}$ & $\begin{array}{l}6.14493 E+00 \\
5.16146 E+\infty 0 \\
5.27477 E+00 \\
4.85679 E+00 \\
5.60651 E+00 \\
5.37593 E+\infty 0 \\
4.94642 E+00 \\
5.15693 E+00 \\
4.78789 E+00 \\
2.07766 E+00 \\
2.32044 E+00 \\
2.44519 E+00 \\
2.78082 E+00\end{array}$ \\
\hline
\end{tabular}

TABLE A-XLIV

FLATTOP-PU CENTRRL AVERAGE CROSS SECTIONS

\begin{tabular}{|c|c|c|c|c|}
\hline Moteriol. & Finion & Copture & Nu & Ironsport \\
\hline $\begin{array}{l}P U-239 \\
P U-240 \\
P U-241 \\
6 R \\
U-235 \\
U-238 \\
N P-237 \\
P U-238 \\
P U-242 \\
P N-241 \\
P-31 \\
P L-27 \\
F E \\
C U\end{array}$ & 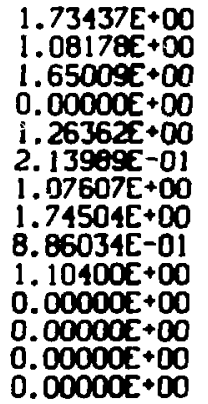 & $\begin{array}{l}7.53247 E-02 \\
1.27913 E-01 \\
1.22761 E-01 \\
3.59834 E-02 \\
1.40772 E-01 \\
7.97057 E-02 \\
3.06322 E-01 \\
2.07221 E-01 \\
9.93085 E-02 \\
4.39557 E-01 \\
2.71004 E-02 \\
4.38786 E-03 \\
8.51140 E-03 \\
2.03081 E-02\end{array}$ & $\begin{array}{l}3.12672 E+00 \\
3.13916 E+00 \\
3.11820 E+00 \\
0.00000 E+00 \\
2.60357 E+00 \\
2.82202 E+00 \\
3.02496 E+00 \\
3.17821 E+00 \\
3.15103 E+00 \\
3.18731 E+00 \\
0.00000 E+00 \\
0.00000 E+00 \\
0.00000 E+00 \\
0.00000 E+00\end{array}$ & $\begin{array}{l}5.30701 E+\infty 0 \\
5.24489 E+00 \\
5.28656 E+\infty 0 \\
3.55857 E++0 \\
5.08549 E+00 \\
5.20139 E+\infty 0 \\
5.55653 E+\infty 0 \\
4.87702 E+00 \\
5.06783 E+00 \\
4.75165 E+\infty 0 \\
2.05680 E+\infty 0 \\
2.26057 E+\infty 0 \\
2.12969 E+\infty 0 \\
2.74736 E+00\end{array}$ \\
\hline
\end{tabular}

TABLE A-XLY

FLATTOP-23 CENTRFL AVERAGE CROSS SECTIONS

\begin{tabular}{|c|c|c|c|c|}
\hline aleriol & Fintion & Coolure & $\mathrm{Nu}$ & Tronoert \\
\hline $\begin{array}{l}-233 \\
-234 \\
-235 \\
238\end{array}$ & $\begin{array}{l}1.95140 E+\infty 0 \\
1.05741 E+00 \\
1.25512 E+00 \\
2.36047 E-01 \\
1.14882 E+\infty\end{array}$ & $\begin{array}{l}7.48318 \varepsilon-02 \\
2.07046 E-01 \\
1.26749 \varepsilon-01 \\
7.26355 E-02 \\
2.67765 E-01\end{array}$ & $\begin{array}{l}2.65766 E+\infty 0 \\
2.65502 \mathrm{\infty}+\infty \\
2.61947 E+\infty 0 \\
2.62191 \mathrm{0}+\infty 0 \\
3.03434 \mathrm{O}+\infty 0\end{array}$ & $\begin{array}{l}+\infty \\
+\infty 0 \\
+\infty 0 \\
+\infty 0\end{array}$ \\
\hline
\end{tabular}

THOLE A-XLYI

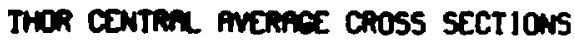

\begin{tabular}{|c|c|}
\hline cint & Eincien \\
\hline $\begin{array}{l}U-230 \\
U-240\end{array}$ & 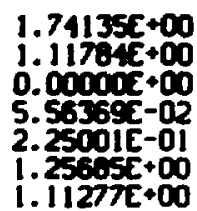 \\
\hline
\end{tabular}
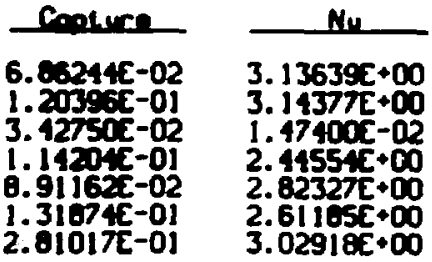

Tronome

5. $363098+00$

5. $30250 .+00$

3. $5 \% 2.15 E+00$

5. $3190 \mathrm{~T}+00$

$5.267955+00$

5. $149965 \cdot 00$

$5.60934 E+00$ 
APPENDIX B

COMPUTER RUN TIMES

Tabulating the present computer run times can prove to be convenient and useful. It can assist the execution of future runs and provide a bast for determining the run time effects of future code changes.

Table B-I is a compilation of the Appendix A computer run times. Table $B-I I$ shows the times required to produce Table II (eigenvalue vs $P_{1}$ ), and Table B-III contains the run times expended to produce Table III (eigenvalue vs $S_{i}$ ). Because we operate in a computer time sharing system, these times are subject to variation depending on the characteristics of other jobs running simultaneously. Such variation could easily be $\pm 20 \%$.

45 
TABLE B-I

COMPUTER RUN TIMES FOR APPENDIX A RESULTS

\begin{tabular}{|c|c|c|c|c|c|c|}
\hline & $\begin{array}{l}\text { SUPERX } \\
\text { Flux and } \\
X \text { Section } \\
\text { (Cray s) }\end{array}$ & $\begin{array}{l}\text { SUPERX } \\
\text { Adjoint } \\
\text { (Cray s) } \\
\end{array}$ & $\begin{array}{l}\text { SUPERB } \\
\text { Diffusion } \\
\text { Theory } \\
\text { Worths } \\
\text { (Cray s) }\end{array}$ & $\begin{array}{c}\text { ONETRAN } \\
\text { F1ux } \\
\text { (CDC7600 s) } \\
\end{array}$ & $\begin{array}{l}\text { ONETRAN } \\
\text { Adjoint } \\
\text { (CDC7600 s) } \\
\end{array}$ & $\begin{array}{l}\text { SUPERB } \\
\text { Transport } \\
\text { Theory } \\
\text { Worths } \\
\text { (Cray s) }\end{array}$ \\
\hline JEZEBEL & 353 & 63 & 60 & 39 & 38 & 66 \\
\hline GODIVA & 178 & 38 & 37 & 40 & 37 & 39 \\
\hline JEZEBEL-23 & 80 & 24 & 29 & 38 & 38 & 25 \\
\hline JEZEBEL-PU & 99 & 25 & 22 & 38 & 38 & 23 \\
\hline BIGTEN & 229 & 65 & 62 & 116 & 132 & 68 \\
\hline FLATTOP-25 & 142 & 19 & 27 & 55 & 111 & 53 \\
\hline FLATTOP-Pu & 157 & 22 & 29 & 96 & 115 & 48 \\
\hline FLATTOP-23 & 93 & 30 & 39 & 100 & 123 & 38 \\
\hline THOR & 94 & 26 & 23 & 54 & 87 & 22 \\
\hline
\end{tabular}

TABLE B-II

ONEDANT CDC-7600 RUN TIMES (s) FOR $P_{i}$ EIGENVALUES (ALL $S_{16}$ )

\begin{tabular}{|c|c|c|c|c|c|c|}
\hline & $P_{0}$ & $P_{1}$ & $\mathrm{P}_{2}$ & $P_{3}$ & $P_{4}$ & $P_{5}$ \\
\hline JEZEBEL & 40 & 54 & 56 & 63 & 69 & 76 \\
\hline GODIVA & 39 & 64 & 65 & 71 & 77 & 83 \\
\hline JEZEBEL-23 & 40 & 61 & 61 & 76 & 76 & 81 \\
\hline JEZEBEL-Pu & 39 & 55 & 56 & 64 & 70 & 76 \\
\hline BIGTEN & 41 & 73 & 80 & 88 & 98 & 107 \\
\hline FLATTOP-25 & 60 & 107 & 125 & 139 & 152 & 166 \\
\hline FLATTOP-Pu & 52 & 104 & 114 & 129 & 141 & 151 \\
\hline FLATTOP-23 & 49 & 88 & 109 & 124 & 136 & 149 \\
\hline THOR & 41 & 68 & 78 & 87 & 100 & 109 \\
\hline
\end{tabular}

TABLE B-III

ONEDANT CDC-7600 RUN TIMES (s) FOR $s_{i}$ EIGENVALUES (ALL $P_{3}$ )

\begin{tabular}{|c|c|c|c|c|c|}
\hline & $s_{4}$ & $\mathrm{~s}_{8}$ & $s_{16}$ & $\mathrm{~S}_{32}$ & $\mathrm{~S}_{48}$ \\
\hline JEZEBEL & 57 & 60 & 63 & 72 & 78 \\
\hline GODIVA & 69 & 71 & 71 & 79 & 90 \\
\hline JEZEBEL-23 & 61 & 62 & 76 & 75 & 83 \\
\hline JEZEBEL-Pu & 58 & 60 & 64 & 72 & 80 \\
\hline BIGTEN & 78 & 83 & 88 & 104 & 118 \\
\hline FLATTOP-25 & 131 & 132 & 139 & 154 & 169 \\
\hline FLATTOP-Pu & 114 & 120 & 129 & 140 & 157 \\
\hline FLATTOP-23 & 112 & 115 & 124 & 139 & 156 \\
\hline THOR & 80 & 82 & 87 & 100 & 111 \\
\hline
\end{tabular}

